\title{
INDECOMPOSABLE DECOMPOSITION OF TENSOR PRODUCTS OF MODULES OVER DRINFELD DOUBLES OF TAFT ALGEBRAS
}

\author{
HUI-XIANG CHEN, HASSEN SULEMAN ESMAEL MOHAMMED, AND HUA SUN
}

\begin{abstract}
In this paper, we study the tensor product structure of category of finite dimensional modules over Drinfeld doubles of Taft Hopf algebras. Tensor product decomposition rules for all finite dimensional indecomposable modules are explicitly given.
\end{abstract}

\section{Introduction}

Representations of a Hopf algebra (up to isomorphism) form a ring, called Green ring, in which the multiplication is given by the tensor product over the base field, and this ring is a commutative ring in the case of the Drinfeld double and any quasitriangular Hopf algebras. The tensor product of representations is an important ingredient in the representation theory of Hopf algebra and quantum groups. In particular, the decomposition of the tensor product of indecomposable modules into direct sum of indecomposables has received enormous attention.

However, in general, very little is known about how a tensor product of two indecomposable modules decomposes into a direct sum of indecomposable modules. There are some results for the decompositions of tensor products of modules over a Hopf algebra or a quantum group. Premet 25] dealt with finite dimensional indecomposable restricted modules for restricted simple 3-dimensional Lie algebra over an algebraically closed field of characteristic $p>2$, and studied the decomposition of tensor product of such modules. Witherspoon 32 studied the Drinfeld double of a finite dimensional group algebra in positive characteristic. She proved that the Green ring of the Drinfeld double of a group algebra decomposes as a product of ideals associated to some subgroups of the original group. Cibils [12] determined all the graded Hopf algebras on a cycle path coalgebra (which are just equal to the generalized Taft algebras (see [9, 15, 26, 31])), and consider the decomposition of the tensor product of two indecomposable modules (see also [14]). Moreover, Cibils also computed the Green ring of the Sweedler 4-dimensional Hopf algebra by generators and relations. Kondo and Saito gave the decomposition of tensor products of modules over the restricted quantum universal enveloping algebra associated to $\mathfrak{s l}_{2}$ in [17. Zhang, Wu, Liu and Chen 33 studied the ring structures of the Grothendieck groups of the Drinfeld doubles of the Taft algebras $H_{n}(q)$. Recently, Chen, Van

Key words and phrases. indecomposable module, tensor product, Hopf algebra, Drinfeld double.

2010 Mathematics Subject Classification. 16E05, 16G99, 16T99. 
Oystaeyen and Zhang [10] computed the Green rings of Taft algebras $H_{n}(q)$, using the decomposition of tensor products of modules given by Cibils [12. $\mathrm{Li}$ and Zhang 21] computed the Green rings of the generalized Taft algebras. When $n=2$, the Taft algebra $H_{2}(-1)$ is exactly the Sweedler's 4-dimensional Hopf algebra $H_{4}$ (see [30, 31]). Chen [8] gave the decomposition of tensor products of modules over $D\left(H_{4}\right)$ and described the Green ring of $D\left(H_{4}\right)$.

We defined a Hopf algebra $H_{n}(1, q)$ by generators and relations in 4] (see the next section), which is isomorphic to the Drinfeld double of a Taft algebra $H_{n}(q)$. We also determined all finite dimensional indecomposable modules over $H_{n}(1, q)$ in [5, 6, 7. Taft algebras belong to the class of biproduct of Nichols algebras as well as "rank one nilpotent type" algebras. The presentation of Drinfeld doubles of rank one pointed Hopf algebras by generators and relations is given in 18, and for general biproduct of Nichols algebras in 28. The Drinfeld doubles of Taft algebras are examples of liftings of quantum planes, whose simple modules, projective covers, primitive idempotents, blocks and quivers are described in [11. Erdmann, Green, Snashall and Taillefer [13] studied the representations of the Drinfeld double of the generalized Taft algebras, and determined the decompositions of the tensor products of two simple modules. They also described the non-projective summands of the tensor products of some other modules, but the projective summands of these tensor products are not described. A natural question is how to determine the decomposition of tensor product of two indecomposable modules over the Drinfeld doubles of the (generalized) Taft algebras.

In this paper, we investigate the indecomposable decompositions of the tensor products of indecomposable modules over the Drinfeld doubles $H_{n}(1, q)$ of the Taft algebras $H_{n}(q)$ for $n>2$. The paper is organized as follow. In Section 2, we recall the structure of $H_{n}(1, q)$, its relation with the small quantum groups and the classification of the indecomposable modules over $H_{n}(1, q)$. In Section 3 , we investigate the tensor product of a simple module with an indecomposable module over $H_{n}(1, q)$, and decompose such tensor products into a direct sum of indecomposable modules, where the decompositions of the tensor products of simple modules are known from [5, 13. In Section 4, we investigate the tensor product of an indecomposable projective module with a non-simple indecomposable module, and decompose such tensor products into a direct sum of indecomposable modules. In Section 5 , we investigate the tensor products of non-simple non-projective indecomposable modules, and decompose such tensor products into a direct sum of indecomposable modules.

\section{Preliminaries}

Throughout, we work over an algebraically closed field $k$. Unless otherwise stated, all algebras, Hopf algebras and modules are defined over $k$; all modules are left modules and finite dimensional; all maps are $k$-linear; dim and $\otimes$ stand for $\operatorname{dim}_{k}$ and $\otimes_{k}$, respectively. For the theory of Hopf algebras and quantum groups, we refer to [16, 23, 30. For the representation theory of finite dimensional algebras, we refer to [2]. Let $\mathbb{Z}$ denote all integers, and $\mathbb{Z}_{n}=\mathbb{Z} / n \mathbb{Z}$ for an integer $n$.

\subsection{Module categories and duality.}


For a finite dimensional algebra $A$, let $\bmod A$ denote the category of finite dimensional $A$-modules. For a module $M$ in $\bmod A$ and a nonnegative integer $s$, let $s M$ denote the direct sum of $s$ copies of $M$. Then $s M=0$ if $s=0$. Let $P(M)$ and $I(M)$ denote the projective cover and the injective envelope of $M$, respectively. Let $\mathrm{l}(M)$ denote the length of $M$, and let $\operatorname{rl}(M)$ denote the Loewy length (=radical length=socle length) of $M$.

Let $H$ be a finite dimensional Hopf algebra. Then $\bmod H$ is a monoidal category 16. 23. If $H$ is a quasitriangular Hopf algebra, then $M \otimes N \cong N \otimes M$ for any $H$-modules $M$ and $N$. It is well known that the Drinfeld double $D(H)$ of a finite dimensional Hopf algebra $H$ is always symmetric (see 22, 24, 27]). For any module $M$ in $\bmod H$, the dual space $M^{*}=\operatorname{Hom}(M, k)$ is also an $H$-module with the action given by

$$
(h \cdot f)(m)=f(S(h) \cdot m), h \in H, f \in M^{*}, m \in M,
$$

where $S$ is the antipode of $H$. It is well known that $(M \otimes N)^{*} \cong N^{*} \otimes M^{*}$ for any $H$-modules $M$ and $N$. If $H$ is quasitriangular, then $S^{2}$ is inner, and so $M^{* *} \cong M$ for any $M \in \bmod H$ (see 22 ). In this case, this gives rise to a duality $(-)^{*}$ from $\bmod H$ to itself.

\subsection{Drinfeld doubles of Taft algebras and small quantum groups.}

The Drinfeld doubles of Taft Hopf algebras and their finite representations were investigated in 4, 5, 6, 7. The representations of pointed Hopf algebras and their Drinfeld doubles were also studied in [19]. The Drinfeld doubles of Taft algebras are closely related with small quantum groups.

First assume that $q \in k$ is an $n^{\text {th }}$ primitive root of unity, $n \geqslant 2$. The Taft Hopf algebra $H_{n}(q)$ is generated by two elements $g$ and $h$ subject to the relations (see [31):

$$
g^{n}=1, \quad h^{n}=0, \quad g h=q h g .
$$

The coalgebra structure and the antipode are determined by

$$
\begin{array}{lll}
\triangle(g)=g \otimes g, & \triangle(h)=h \otimes g+1 \otimes h, & \varepsilon(g)=1, \\
\varepsilon(h)=0, & S(g)=g^{-1}=g^{n-1}, & S(h)=-q^{-1} g^{n-1} h .
\end{array}
$$

Note that $\operatorname{dim} H_{n}(q)=n^{2}$, and $\left\{g^{i} h^{j} \mid 0 \leqslant i, j \leqslant n-1\right\}$ forms a $k$-basis for $H_{n}(q)$. When $n=2, H_{2}(q)$ is exactly the Sweedler 4-dimensional Hopf algebra. The Drinfeld double $D\left(H_{n}(q)\right)$ can be described as follows.

Let $p \in k$. Then one can define an $n^{4}$-dimensional Hopf algebra $H_{n}(p, q)$, which is generated as an algebra by $a, b, c$ and $d$ subject to the relations:

$$
\begin{aligned}
& b a=q a b, \quad d b=q b d, \quad c a=q a c, \quad d c=q c d, \quad b c=c b, \\
& a^{n}=0, \quad b^{n}=1, \quad c^{n}=1, \quad d^{n}=0, \quad d a-q a d=p(1-b c) .
\end{aligned}
$$

The coalgebra structure and the antipode are given by

$$
\begin{array}{lll}
\triangle(a)=a \otimes b+1 \otimes a, & \varepsilon(a)=0, & S(a)=-a b^{-1}=-a b^{n-1}, \\
\triangle(b)=b \otimes b, & \varepsilon(b)=1, & S(b)=b^{-1}=b^{n-1}, \\
\triangle(c)=c \otimes c, & \varepsilon(c)=1, & S(c)=c^{-1}=c^{n-1}, \\
\triangle(d)=d \otimes c+1 \otimes d, & \varepsilon(d)=0, & S(d)=-d c^{-1}=-d c^{n-1} .
\end{array}
$$


$H_{n}(p, q)$ has a $k$-basis $\left\{a^{i} b^{j} c^{l} d^{k} \mid 0 \leqslant i, j, l, k \leqslant n-1\right\}$, and is not semisimple. If $p \neq 0$, then $H_{n}(p, q)$ is isomorphic to $D\left(H_{n}(q)\right)$ as a Hopf algebra. In particular, we have $H_{n}(p, q) \cong H_{n}(1, q) \cong D\left(H_{n}(q)\right)$ for any $p \neq 0$. For the details, the reader is directed to [4, 5]. When $n=2$ and $p=0, H_{2}(0, q)$ is exactly the Hopf algebra $\overline{\mathcal{A}}$ in 20 .

Next assume that $q \in k$ is an $m^{\text {th }}$ primitive root of unity with $m>2$. Let $U_{q}$ be the quantum enveloping algebra $U_{q}\left(\mathfrak{s l}_{2}\right)$ of Lie algebra $\mathfrak{s l}_{2}$ described in [16. Then $U_{q}$ is a Hopf algebra. Let $n=m$ if $m$ is odd, and $n=\frac{m}{2}$ if $m$ is even. Let $I$ be the ideal of $U_{q}$ generated by $E^{n}, F^{n}$ and $K^{n}-1$. Then $I$ is a Hopf ideal of $U_{q}$, and hence one gets a quotient Hopf algebra $\bar{U}_{q}:=U_{q} / I$, the small quantum group. Note that $q^{2}$ is an $n^{\text {th }}$ primitive root of unity. Hence one can form a Hopf algebra $H_{n}\left(1, q^{2}\right)$ as above. Then a straightforward verification shows that there is a Hopf algebra epimorphism $\phi: H_{n}\left(1, q^{2}\right) \rightarrow \bar{U}_{q}$ determined by (see [1, Proposition 4.5])

$$
\phi(a)=E, \phi(b)=K, \phi(c)=K, \phi(d)=q^{-2}\left(q-q^{-1}\right) F K .
$$

Let $C$ be the group of central group-like elements in $H_{n}\left(1, q^{2}\right)$. Then $\operatorname{Ker}(\phi)=$ $(k C)^{+} H_{n}\left(1, q^{2}\right)$. Moreover, $H_{n}\left(1, q^{2}\right) \cong k C \otimes H_{n}\left(1, q^{2}\right) /(k C)^{+} H_{n}\left(1, q^{2}\right) \cong k C \otimes \bar{U}_{q}$ as algebras if $n$ is odd. Chari and Premet in [3] worked out all indecomposable modules for $\bar{U}_{q}$ when $m$ is odd. Hence the indecomposable modules over $H_{n}\left(1, q^{2}\right)$ can be induced from those over $\bar{U}_{q}$ for any odd $n$. Note that Suter worked out all indecomposable modules for a slightly different version of $\bar{U}_{q}$ in [29].

\subsection{Indecomposable modules over $H_{n}(1, q)$.}

Let $J:=\operatorname{rad}\left(H_{n}(1, q)\right)$ stand for the Jacobson radical of $H_{n}(1, q)$. Then $J^{3}=0$ by [7, Corollary 2.4]. This means that the Loewy length of $H_{n}(1, q)$ is 3 . In order to study the tensor products of modules over $H_{n}(1, q)$, we need first to give the structures of all finite dimensional indecomposable $H_{n}(1, q)$-modules. We will follow the notations of [7]. Unless otherwise stated, all modules are modules over $H_{n}(1, q)$ in what follows.

From [7, we know that the socle series and the radical series of an indecomposable module coincide. We list all indecomposable modules according to the Loewy length. There are $n^{2}$ simple modules up to isomorphism.

Simple modules: $V(l, r), 1 \leqslant l \leqslant n, r \in \mathbb{Z}_{n} . \quad V(l, r)$ has a standard $k$-basis $\left\{v_{i} \mid 1 \leqslant i \leqslant l\right\}$ such that

$$
\begin{aligned}
& a v_{i}=\left\{\begin{array}{ll}
v_{i+1}, & 1 \leqslant i<l, \\
0, & i=l,
\end{array} \quad d v_{i}= \begin{cases}0, & i=1, \\
\alpha_{i-1}(l) v_{i-1}, & 1<i \leqslant l,\end{cases} \right. \\
& b v_{i}=q^{r+i-1} v_{i}, 1 \leqslant i \leqslant l, \quad c v_{i}=q^{i-r-l} v_{i}, 1 \leqslant i \leqslant l,
\end{aligned}
$$

where $\alpha_{i}(l)=(i)_{q}\left(1-q^{i-l}\right)$ for $1 \leqslant i<l \leqslant n$. The simple modules $V(n, r), r \in \mathbb{Z}_{n}$, are projective and injective.

Projective modules of Loewy length 3: Let $P(l, r)$ be the projective cover of $V(l, r)$, $1 \leqslant l<n, r \in \mathbb{Z}_{n}$. Then $P(l, r)$ is the injective envelope of $V(l, r)$ as well. $P(l, r)$ 
has a standard $k$-basis $\left\{v_{i} \mid 1 \leqslant i \leqslant 2 n\right\}$ such that

$$
\begin{gathered}
a v_{i}= \begin{cases}v_{i+1}, & 1 \leqslant i<n \text { or } n+1 \leqslant i<2 n, \\
0, & i=n \text { or } 2 n,\end{cases} \\
b v_{i}=\left\{\begin{array}{ll}
q^{r+i-1} v_{i}, & 1 \leqslant i \leqslant n, \\
q^{r+l+i-1} v_{i}, & n+1 \leqslant i \leqslant 2 n,
\end{array} \quad c v_{i}= \begin{cases}q^{i-l-r} v_{i}, & 1 \leqslant i \leqslant n, \\
q^{i-r} v_{i}, & n+1 \leqslant i \leqslant 2 n,\end{cases} \right. \\
d v_{i}= \begin{cases}q^{i-1} v_{2 n-l+i-1}, & i=1 \text { or } l+1, \\
q^{i-1} v_{2 n-l+i-1}+\alpha_{i-1}(l) v_{i-1}, & 1<i \leqslant l, \\
\alpha_{i-l-1}(n-l) v_{i-1}, & l+1<i \leqslant n, \\
0, & i=n+1 \text { or } 2 n-l+1, \\
\alpha_{i-n-1}(n-l) v_{i-1}, & n+1<i \leqslant 2 n-l, \\
\alpha_{i-2 n+l-1}(l) v_{i-1}, & 2 n-l+1<i \leqslant 2 n .\end{cases}
\end{gathered}
$$

Moreover, we have (see [7])

$$
\begin{aligned}
& \operatorname{soc} P(l, r)=\operatorname{rad}^{2} P(l, r) \cong P(l, r) / \operatorname{rad} P(l, r)=P(l, r) / \operatorname{soc}^{2} P(l, r) \cong V(l, r), \\
& \operatorname{soc}^{2} P(l, r) / \operatorname{soc}(P(l, r))=\operatorname{rad} P(l, r) / \operatorname{rad}^{2} P(l, r) \cong 2 V(n-l, r+l) .
\end{aligned}
$$

For non-isomorphic indecomposable modules with Loewy length 2, we list them according to the lengths and the co-lengths of their socles. We say that an indecomposable module $M$ with $\operatorname{rl}(M)=2$ is of $(s, t)$-type if $\mathrm{l}(M / \operatorname{soc}(M))=s$ and $\mathrm{l}(\operatorname{soc}(M))=t$. By [7], if $M$ is of $(s, t)$-type, then $s=t+1$, or $s=t$, or $s=t-1$. Note that $M$ is a string module for $s=t+1$ and $s=t-1 ; M$ is a band module for $s=t$.

String modules: The indecomposable modules of $(s+1, s)$-type are given by the syzygy functor $\Omega$. Let $V(l, r)$ be the simple modules given above, $1 \leqslant l<n, r \in \mathbb{Z}_{n}$. Then the minimal projective resolutions of $V(l, r)$ are given by

$$
\cdots \rightarrow 4 P(n-l, r+l) \rightarrow 3 P(l, r) \rightarrow 2 P(n-l, r+l) \rightarrow P(l, r) \rightarrow V(l, r) \rightarrow 0 .
$$

By these resolutions, one can describe the structure of $\Omega^{s} V(l, r), s \geqslant 1$ (see [7]). The string module $\Omega^{s} V(l, r)$ is of $(s+1, s)$-type. The indecomposable modules of $(s, s+1)$-type are given by the cosyzygy functor $\Omega^{-1}$. For $1 \leqslant l<n$ and $r \in \mathbb{Z}_{n}$, the minimal injective resolutions of $V(l, r)$ are given by

$$
0 \rightarrow V(l, r) \rightarrow P(l, r) \rightarrow 2 P(n-l, r+l) \rightarrow 3 P(l, r) \rightarrow 4 P(n-l, r+l) \rightarrow \cdots .
$$

By these resolutions, one can describe the structure of $\Omega^{-s} V(l, r), s \geqslant 1$ (see [7]). The string module $\Omega^{-s} V(l, r)$ is of $(s, s+1)$-type.

Let $1 \leqslant l<n, r \in \mathbb{Z}_{n}$ and $s \geqslant 1$. If $s$ is odd, then we have

$$
\begin{gathered}
\operatorname{soc}\left(\Omega^{s} V(l, r)\right) \cong \Omega^{-s} V(l, r) / \operatorname{soc}\left(\Omega^{-s} V(l, r)\right) \cong s V(l, r), \\
\operatorname{soc}\left(\Omega^{-s} V(l, r)\right) \cong \Omega^{s} V(l, r) / \operatorname{soc}\left(\Omega^{s} V(l, r)\right) \cong(s+1) V(n-l, r+l) .
\end{gathered}
$$

If $s$ is even, then we have

$$
\begin{gathered}
\operatorname{soc}\left(\Omega^{s} V(l, r)\right) \cong \Omega^{-s} V(l, r) / \operatorname{soc}\left(\Omega^{-s} V(l, r)\right) \cong s V(n-l, r+l), \\
\operatorname{soc}\left(\Omega^{-s} V(l, r)\right) \cong \Omega^{s} V(l, r) / \operatorname{soc}\left(\Omega^{s} V(l, r)\right) \cong(s+1) V(l, r) .
\end{gathered}
$$


Band modules: The indecomposable modules of $(s, s)$-type can be described as follows. Let $\mathbb{P}^{1}(k)$ be the projective 1 -space over $k$. $\mathbb{P}^{1}(k)$ can be regarded as the set of all 1-dimensional subspaces of $k^{2}$. Let $\infty$ be a symbol with $\infty \notin k$ and let $\bar{k}=k \cup\{\infty\}$. Then there is a bijection between $\bar{k}$ and $\mathbb{P}^{1}(k): \alpha \mapsto L(\alpha, 1)$, $\infty \mapsto L(1,0)$, where $\alpha \in k$ and $L(\alpha, \beta)$ denotes the 1-dimensional subspace of $k^{2}$ with basis $(\alpha, \beta)$ for any $0 \neq(\alpha, \beta) \in k^{2}$. In the following, we regard $\mathbb{P}^{1}(k)=\bar{k}$.

If $M$ is of $(s, s)$-type then $M \cong M_{s}(l, r, \eta)$, where $1 \leqslant l<n, r \in \mathbb{Z}_{n}$ and $\eta \in \mathbb{P}^{1}(k)$ (see [7]). The indecomposable module $M_{1}(l, r, \infty), 1 \leqslant l<n, r \in \mathbb{Z}_{n}$, has a standard basis $\left\{v_{1}, v_{2}, \cdots, v_{n}\right\}$ such that

$$
\begin{aligned}
& a v_{i}=\left\{\begin{array}{ll}
0, \quad i=n-l \text { or } n, \\
v_{i+1}, \quad \text { otherwise },
\end{array} \quad d v_{i}= \begin{cases}v_{n}, & i=1, \\
\alpha_{i-1}(n-l) v_{i-1}, & 1<i \leqslant n-l, \\
0, & i=n-l+1, \\
\alpha_{i-n+l-1}(l) v_{i-1}, & n-l+1<i \leqslant n,\end{cases} \right. \\
& b v_{i}=q^{r+l+i-1} v_{i},
\end{aligned}
$$

The indecomposable module $M_{1}(l, r, \eta), 1 \leqslant l<n, r \in \mathbb{Z}_{n}, \eta \in k$, has a standard basis $\left\{v_{1}, v_{2}, \cdots, v_{n}\right\}$ with the action given by

$$
\begin{aligned}
& a v_{i}=\left\{\begin{array}{ll}
v_{i+1}, & 1 \leqslant i<n, \\
0, & i=n,
\end{array} \quad d v_{i}= \begin{cases}\eta q^{l} v_{n}, & i=1, \\
\alpha_{i-1}(n-l) v_{i-1}, & 1<i \leqslant n-l, \\
0, & i=n-l+1, \\
\alpha_{i-n+l-1}(l) v_{i-1}, & n-l+1<i \leqslant n,\end{cases} \right. \\
& b v_{i}=q^{r+l+i-1} v_{i},
\end{aligned}
$$

Then the band modules $M_{s}(l, r, \eta)$ are determined recursively by the almost split sequences

$$
0 \rightarrow M_{s}(l, r, \eta) \rightarrow M_{s-1}(l, r, \eta) \oplus M_{s+1}(l, r, \eta) \rightarrow M_{s}(l, r, \eta) \rightarrow 0,
$$

where $s \geqslant 1, M_{0}(l, r, \eta)=0,1 \leqslant l<n, r \in \mathbb{Z}_{n}$ and $\eta \in \mathbb{P}^{1}(k)$ (see [6, 7]). $M_{s}(l, r, \eta)$ also can be constructed recursively by using pullback (see [7, pp. 28232824]). $M_{s}(l, r, \eta)$ is a submodule of $s P(l, r)$ and a quotient module of $s P(n-l, r+l)$, and there is an exact sequence

$$
0 \rightarrow M_{s}(l, r, \eta) \hookrightarrow s P(l, r) \rightarrow M_{s}\left(n-l, r+l,-\eta q^{l}\right) \rightarrow 0 .
$$

Hence $\Omega M_{s}(l, r, \eta) \cong \Omega^{-1} M_{s}(l, r, \eta) \cong M_{s}\left(n-l, r+l,-\eta q^{l}\right)$. Moreover, for any $1 \leqslant i<s, M_{s}(l, r, \eta)$ contains a unique submodule of $(i, i)$-type, which is isomorphic to $M_{i}(l, r, \eta)$ and the quotient module of $M_{s}(l, r, \eta)$ modulo the submodule of $(i, i)$ type is isomorphic to $M_{s-i}(l, r, \eta)$. Hence there is an exact sequence of modules

$$
0 \rightarrow M_{i}(l, r, \eta) \hookrightarrow M_{s}(l, r, \eta) \rightarrow M_{s-i}(l, r, \eta) \rightarrow 0 .
$$

Erdmann, Green, Snashall and Taillefer studied the representations of the Drinfeld double $D\left(\Lambda_{n, d}\right)$ of the generalized Taft algebras $\Lambda_{n, d}$ in 13 . In case $d=n, \Lambda_{n, n}$ is the $n^{2}$-dimensional Taft Hopf algebra. For this reason, $\Lambda_{n, d}$ is called a generalized Taft algebra in [9, 15]. Moreover, $D\left(\Lambda_{n, n}\right) \cong H_{n}(1, q)$ as Hopf algebras. Hence one also can get all indecomposable modules over $H_{n}(1, q)$ from 13 . In this case, $V(l, r)$ is the simple module $L(1-2 r-l, r)$, and the band modules $M_{s}(l, r, 0)$ and $M_{s}(l, r, \infty)$ are string modules of even length in [13]. 
Throughout the following, let $n$ be a fixed positive integer with $n>2$, and $q \in k$ an $n^{\text {th }}$ primitive root of unity. Let $P(n, r)=V(n, r)$ and $\Omega^{0} V(l, r)=V(l, r)$ for all $1 \leqslant l<n$ and $r \in \mathbb{Z}_{n}$, and let $\alpha \infty=\infty \alpha=\infty$ for any $0 \neq \alpha \in k$. Let $\mathcal{M}$ denote the category of finite dimensional modules over $H_{n}(1, q)$.

\section{Tensor product of a simple module with a module}

In this section, we investigate the tensor product of a simple module with an indecomposable module. Throughout the following, unless otherwise stated, a module means a module over $H_{n}(1, q)$, and an isomorphism means a module isomorphism.

Note that $M \otimes N \cong N \otimes M$ for any modules $M$ and $N$ since $H_{n}(1, q)$ is a quasitriangular Hopf algebra. For any $t \in \mathbb{Z}$, let $c(t):=\left[\frac{t+1}{2}\right]$ be the integer part of $\frac{t+1}{2}$. That is, $c(t)$ is the maximal integer with respect to $c(t) \leqslant \frac{t+1}{2}$. Then $c(t)+c(t-1)=t$.

\subsection{Tensor product of two simple modules.}

The decomposition of the tensor product of two simple modules has been determined in [5, 13. We gave the decomposition of the tensor product $V(l, r) \otimes V\left(l^{\prime}, r^{\prime}\right)$ for $l+l^{\prime} \leqslant n+1$, and described the socle of $V(l, r) \otimes V\left(l^{\prime}, r^{\prime}\right)$ for $l+l^{\prime}>n+1$ in [5]. Erdmann, Green, Snashall and Taillefer described the decomposition of the tensor product of any two simple modules for the Drinfeld double of the generalized Taft algebras $\Lambda_{n, d}$ in [13. Putting $d=n$ in [13], one can get the decomposition of $V(l, r) \otimes V\left(l^{\prime}, r^{\prime}\right)$ for $l+l^{\prime}>n+1$ (also for $\left.l+l^{\prime} \leqslant n+1\right)$.

Convention: If $\oplus_{l \leqslant i \leqslant m} M_{i}$ is a term in a decomposition of a module, then it disappears when $l>m$. For instance, in the decomposition of the following Proposition 3.1 (2), the term $\oplus_{t+1 \leqslant i \leqslant l-1} V\left(l+l^{\prime}-1-2 i, r+r^{\prime}+i\right)$ disappears when $l^{\prime}=n$, or equivalently $t=l-1$.

Proposition 3.1. Let $1 \leqslant l \leqslant l^{\prime} \leqslant n$ and $r, r^{\prime} \in \mathbb{Z}_{n}$.

(1) If $l+l^{\prime} \leqslant n+1$, then $V(l, r) \otimes V\left(l^{\prime}, r^{\prime}\right) \cong \oplus_{i=0}^{l-1} V\left(l+l^{\prime}-1-2 i, r+r^{\prime}+i\right)$. In particular, $V(1, r) \otimes V\left(l^{\prime}, r^{\prime}\right) \cong V\left(l^{\prime}, r+r^{\prime}\right)$ for all $1 \leqslant l^{\prime} \leqslant n$ and $r, r^{\prime} \in \mathbb{Z}_{n}$.

(2) If $t=l+l^{\prime}-(n+1) \geqslant 0$, then

$$
\begin{aligned}
V(l, r) \otimes V\left(l^{\prime}, r^{\prime}\right) \cong & \left(\oplus_{i=c(t)}^{t} P\left(l+l^{\prime}-1-2 i, r+r^{\prime}+i\right)\right) \\
& \oplus\left(\oplus_{t+1 \leqslant i \leqslant l-1} V\left(l+l^{\prime}-1-2 i, r+r^{\prime}+i\right)\right) .
\end{aligned}
$$

Proof. It follows from [5, Theorem 3.1] and [13, Theorem 4.1].

By the Fundamental Theorem of Hopf modules (see [23]), $M \otimes P$ is projective for any projective module $P$ and any module $M$. Thus, one gets the following corollary.

Corollary 3.2. The subcategory consisting of semisimple modules and projective modules in $\mathcal{M}$ is a monoidal subcategory of $\mathcal{M}$.

\subsection{Tensor product of a simple module with a projective module.}

In this subsection, we determine the tensor product $V(l, r) \otimes P\left(l^{\prime}, r^{\prime}\right)$ of a simple module with an indecomposable projective module. As pointed out in the last subsection, $V(l, r) \otimes P\left(l^{\prime}, r^{\prime}\right)$ is projective, and so it is also injective. Thus, it is 
enough to determine the socle of $V(l, r) \otimes P\left(l^{\prime}, r^{\prime}\right)$. If $M$ is a submodule of the socle of $V(l, r) \otimes P\left(l^{\prime}, r^{\prime}\right)$, then $P(M)(\cong I(M))$ is isomorphic to a submodule of $V(l, r) \otimes P\left(l^{\prime}, r^{\prime}\right)$. We will manage to find a submodule $U$ of the socle of $V(l, r) \otimes$ $P\left(l^{\prime}, r^{\prime}\right)$ such that $P(U)$ and $V(l, r) \otimes P\left(l^{\prime}, r^{\prime}\right)$ have the same dimension. In this case, $V(l, r) \otimes P\left(l^{\prime}, r^{\prime}\right) \cong P(U)$. In the following, we will also use the fact that if a projective module $P$ is isomorphic to a submodule of a quotient module of a module $M$, then $P$ is isomorphic to a summand of $M$.

Theorem 3.3. Let $1 \leqslant l, l^{\prime}<n$ and $r, r^{\prime} \in \mathbb{Z}_{n}$. Assume that $l+l^{\prime} \leqslant n$. Let $l_{1}=\min \left\{l, l^{\prime}\right\}$. Then

$$
\begin{aligned}
V(l, r) \otimes P\left(l^{\prime}, r^{\prime}\right) \cong & \left(\oplus_{i=0}^{l_{1}-1} P\left(l+l^{\prime}-1-2 i, r+r^{\prime}+i\right)\right) \\
& \oplus\left(\oplus_{c\left(l+l^{\prime}-1\right) \leqslant i \leqslant l-1} 2 P\left(n+l+l^{\prime}-1-2 i, r+r^{\prime}+i\right)\right) .
\end{aligned}
$$

Proof. We first assume that $l \leqslant l^{\prime}$ and let $V=V(l, r) \otimes P\left(l^{\prime}, r^{\prime}\right)$. Then $V_{1}:=$ $V(l, r) \otimes \operatorname{soc}\left(P\left(l^{\prime}, r^{\prime}\right)\right)$ is a submodule of $V$. Since $\operatorname{soc}\left(P\left(l^{\prime}, r^{\prime}\right)\right) \cong V\left(l^{\prime}, r^{\prime}\right)$, it follows from Proposition 3.1 (1) that $V_{1} \cong V(l, r) \otimes V\left(l^{\prime}, r^{\prime}\right) \cong \oplus_{i=0}^{l-1} V\left(l+l^{\prime}-1-2 i, r+r^{\prime}+i\right)$. Hence $P\left(V_{1}\right)$ can be embedded into $V$ as a submodule. Now we have $P\left(V_{1}\right) \cong$ $\oplus_{i=0}^{l-1} P\left(V\left(l+l^{\prime}-1-2 i, r+r^{\prime}+i\right)\right) \cong \oplus_{i=0}^{l-1} P\left(l+l^{\prime}-1-2 i, r+r^{\prime}+i\right)$. Since $1 \leqslant l+l^{\prime}-1-2 i \leqslant n-1$ for all $0 \leqslant i \leqslant l-1, \operatorname{dim}\left(P\left(l+l^{\prime}-1-2 i, i\right)\right)=2 n$, and so $\operatorname{dim}\left(P\left(V_{1}\right)\right)=2 n l=\operatorname{dim}(V)$. This implies

$$
V(l, r) \otimes P\left(l^{\prime}, r^{\prime}\right) \cong \oplus_{i=0}^{l-1} P\left(l+l^{\prime}-1-2 i, r+r^{\prime}+i\right) .
$$

Next, assume that $l^{\prime}<l$. Applying $V(l, r) \otimes$ to the exact sequence $0 \rightarrow V\left(l^{\prime}, r^{\prime}\right) \rightarrow$ $P\left(l^{\prime}, r^{\prime}\right) \rightarrow \Omega^{-1} V\left(l^{\prime}, r^{\prime}\right) \rightarrow 0$, one gets anther exact sequence

$$
0 \rightarrow V(l, r) \otimes V\left(l^{\prime}, r^{\prime}\right) \rightarrow V(l, r) \otimes P\left(l^{\prime}, r^{\prime}\right) \rightarrow V(l, r) \otimes \Omega^{-1} V\left(l^{\prime}, r^{\prime}\right) \rightarrow 0 .
$$

Note that $l \leqslant n-l^{\prime}$ and $l+n-l^{\prime}-(n+1)=l-l^{\prime}-1 \geqslant 0$. By $\operatorname{soc}\left(\Omega^{-1} V\left(l^{\prime}, r^{\prime}\right)\right) \cong$ $2 V\left(n-l^{\prime}, r^{\prime}+l^{\prime}\right)$ and Proposition 3.1(2), we have

$$
\begin{aligned}
V(l, r) \otimes \operatorname{soc}\left(\Omega^{-1} V\left(l^{\prime}, r^{\prime}\right)\right) & \cong 2 V(l, r) \otimes V\left(n-l^{\prime}, r^{\prime}+l^{\prime}\right) \\
& \cong\left(\oplus_{i=c\left(l-l^{\prime}-1\right)}^{l-l^{\prime}-1} 2 P\left(l+n-l^{\prime}-1-2 i, r+r^{\prime}+l^{\prime}+i\right)\right) \\
& \oplus\left(\oplus_{i=l-l l^{\prime}}^{l-1} 2 V\left(l+n-l^{\prime}-1-2 i, r+r^{\prime}+l^{\prime}+i\right)\right) \\
& \cong\left(\oplus_{i=c}^{l-1}\left(l+l^{\prime}-1\right) 2 P\left(n+l+l^{\prime}-1-2 i, r+r^{\prime}+i\right)\right) \\
& \oplus\left(\oplus_{i=l}^{l+l^{\prime}-1} 2 V\left(n+l+l^{\prime}-1-2 i, r+r^{\prime}+i\right)\right) .
\end{aligned}
$$

Since $\oplus_{i=c\left(l+l^{\prime}-1\right)}^{l-1} 2 P\left(n+l+l^{\prime}-1-2 i, r+r^{\prime}+i\right)$ is projective and injective, it follows that there is an epimorphism

$$
\phi: V:=V(l, r) \otimes P\left(l^{\prime}, r^{\prime}\right) \rightarrow \oplus_{i=c\left(l+l^{\prime}-1\right)}^{l-1} 2 P\left(n+l+l^{\prime}-1-2 i, r+r^{\prime}+i\right)
$$

such that $\operatorname{Ker}(\phi)$ contains a submodule isomorphic to $V(l, r) \otimes V\left(l^{\prime}, r^{\prime}\right)$. Hence $V=\operatorname{Ker}(\phi) \oplus P$, where $P$ is a submodule of $V$ with $P \cong \oplus_{i=c\left(l+l^{\prime}-1\right)}^{l-1} 2 P(n+l+$ $\left.l^{\prime}-1-2 i, r+r^{\prime}+i\right)$, and $\operatorname{Ker}(\phi)$ contains a submodule $V_{1}$ with $V_{1} \cong V(l, r) \otimes$ $V\left(l^{\prime}, r^{\prime}\right)$. By Proposition 3.1 (1), $V_{1} \cong \oplus_{i=0}^{l^{\prime}-1} V\left(l+l^{\prime}-1-2 i, r+r^{\prime}+i\right)$. Hence $\operatorname{soc}(V)=\operatorname{soc}(\operatorname{Ker}(\phi)) \oplus \operatorname{soc}(P) \supseteq \operatorname{soc}\left(V_{1}\right) \oplus \operatorname{soc}(P) \cong\left(\oplus_{i=0}^{l^{\prime}-1} V\left(l+l^{\prime}-1-2 i, r+\right.\right.$ $\left.\left.r^{\prime}+i\right)\right) \oplus\left(\oplus_{i=c\left(l+l^{\prime}-1\right)}^{l-1} 2 V\left(n+l+l^{\prime}-1-2 i, r+r^{\prime}+i\right)\right)=: U$. Thus, $P(U)$ is 
isomorphic to a submodule of $V$. Then a straightforward computation shows that $\operatorname{dim} P(U)=2 n l=\operatorname{dim}(V)$, and so

$$
\begin{aligned}
V(l, r) \otimes P\left(l^{\prime}, r^{\prime}\right) \cong P(U) \cong & \left(\oplus_{i=0}^{l^{\prime}-1} P\left(l+l^{\prime}-1-2 i, r+r^{\prime}+i\right)\right) \\
& \oplus\left(\oplus_{i=c\left(l+l^{\prime}-1\right)}^{l-1} 2 P\left(n+l+l^{\prime}-1-2 i, r+r^{\prime}+i\right)\right) .
\end{aligned}
$$

This completes the proof.

Corollary 3.4. Let $1 \leqslant l \leqslant n$ and $r, r^{\prime} \in \mathbb{Z}_{n}$. Then $V(1, r) \otimes P\left(l, r^{\prime}\right) \cong P\left(l, r+r^{\prime}\right)$.

Proof. It is follows from Theorem 3.3 for $1 \leqslant l<n$, and Proposition 3.1(1) for $l=n$.

Theorem 3.5. Let $1 \leqslant l, l^{\prime} \leqslant n$ with $l^{\prime} \neq n$ and $r, r^{\prime} \in \mathbb{Z}_{n}$. Assume that $t=l+l^{\prime}-(n+1) \geqslant 0$ and let $l_{1}=\min \left\{l, l^{\prime}\right\}$. Then

$$
\begin{aligned}
V(l, r) \otimes P\left(l^{\prime}, r^{\prime}\right) \cong & \left(\oplus_{i=c(t)}^{t} 2 P\left(l+l^{\prime}-1-2 i, r+r^{\prime}+i\right)\right) \\
& \oplus\left(\oplus_{t+1 \leqslant i \leqslant l_{1}-1} P\left(l+l^{\prime}-1-2 i, r+r^{\prime}+i\right)\right) \\
& \oplus\left(\oplus_{c\left(l+l^{\prime}-1\right) \leqslant i \leqslant l-1} 2 P\left(n+l+l^{\prime}-1-2 i, r+r^{\prime}+i\right)\right) .
\end{aligned}
$$

Proof. By Proposition 3.1(1) and Corollary 3.4, we only need to consider the case of $r=r^{\prime}=0$.

First assume that $l \leqslant l^{\prime}$. Then $t<l-1$ by $l^{\prime}<n$. We have an exact sequence

$$
0 \rightarrow V(l, 0) \otimes \Omega V\left(l^{\prime}, 0\right) \rightarrow V(l, 0) \otimes P\left(l^{\prime}, 0\right) \rightarrow V(l, 0) \otimes V\left(l^{\prime}, 0\right) \rightarrow 0 .
$$

By Proposition $3.1(2), \oplus_{i=c(t)}^{t} P\left(l+l^{\prime}-1-2 i, i\right)$ is isomorphic to a summand of $V(l, 0) \otimes V\left(l^{\prime}, 0\right)$. Hence there is a module epimorphism

$$
\phi: V:=V(l, 0) \otimes P\left(l^{\prime}, 0\right) \rightarrow \oplus_{i=c(t)}^{t} P\left(l+l^{\prime}-1-2 i, i\right)
$$

such that $\operatorname{Ker}(\phi)$ contains a submodule isomorphic to $V(l, 0) \otimes \Omega V\left(l^{\prime}, 0\right)$. Note that $V(l, 0) \otimes \Omega V\left(l^{\prime}, 0\right) \supseteq V(l, 0) \otimes \operatorname{soc}\left(\Omega V\left(l^{\prime}, 0\right)\right) \cong V(l, 0) \otimes V\left(l^{\prime}, 0\right)$. Thus, again by Proposition $3.1(2)$, an argument similar to Theorem 3.3 shows that $\operatorname{soc}(V)$ contains a submodule $U$ with

$$
\begin{aligned}
U & \cong \operatorname{soc}\left(V(l, 0) \otimes V\left(l^{\prime}, 0\right)\right) \oplus\left(\oplus_{i=c(t)}^{t} V\left(l+l^{\prime}-1-2 i, i\right)\right) \\
& \cong\left(\oplus_{i=c(t)}^{t} 2 V\left(l+l^{\prime}-1-2 i, i\right)\right) \oplus\left(\oplus_{i=t+1}^{l-1} V\left(l+l^{\prime}-1-2 i, i\right)\right) .
\end{aligned}
$$

Thus, $P(U)$ is isomorphic to a submodule of $V$. Then a straightforward computation shows that $\operatorname{dim} P(U)=2 n l=\operatorname{dim}(V)$. It follows that

$$
V \cong P(U) \cong\left(\oplus_{i=c(t)}^{t} 2 P\left(l+l^{\prime}-1-2 i, i\right)\right) \oplus\left(\oplus_{i=t+1}^{l-1} P\left(l+l^{\prime}-1-2 i, i\right)\right) .
$$

Now assume that $l^{\prime}<l$. We have two exact sequences

$$
\begin{gathered}
0 \rightarrow V(l, 0) \otimes V\left(l^{\prime}, 0\right) \rightarrow V(l, 0) \otimes P\left(l^{\prime}, 0\right) \rightarrow V(l, 0) \otimes \Omega^{-1} V\left(l^{\prime}, 0\right) \rightarrow 0, \\
0 \rightarrow 2 V(l, 0) \otimes V\left(n-l^{\prime}, l^{\prime}\right) \rightarrow V(l, 0) \otimes \Omega^{-1} V\left(l^{\prime}, 0\right) \rightarrow V(l, 0) \otimes V\left(l^{\prime}, 0\right) \rightarrow 0 .
\end{gathered}
$$

Note that $n-l^{\prime}<l$ and $l+n-l^{\prime}-(n+1)=l-l^{\prime}-1 \geqslant 0$. By Proposition 3.1(2), $2 V(l, 0) \otimes V\left(n-l^{\prime}, l^{\prime}\right)$ contains a summand isomorphic to

$$
\oplus_{i=c\left(l-l^{\prime}-1\right)}^{l-l^{\prime}-1} 2 P\left(l+n-l^{\prime}-1-2 i, l^{\prime}+i\right) \cong \oplus_{i=c\left(l+l^{\prime}-1\right)}^{l-1} 2 P\left(n+l+l^{\prime}-1-2 i, i\right),
$$


and $V(l, 0) \otimes V\left(l^{\prime}, 0\right)$ contains a summand isomorphic to $\oplus_{i=c(t)}^{t} P\left(l+l^{\prime}-1-2 i, i\right)$. It follows from the last exact sequence that $V(l, 0) \otimes \Omega^{-1} V\left(l^{\prime}, 0\right)$ contains a projective summand $P$ with

$$
P \cong\left(\oplus_{i=c(t)}^{t} P\left(l+l^{\prime}-1-2 i, i\right)\right) \oplus\left(\oplus_{i=c\left(l+l^{\prime}-1\right)}^{l-1} 2 P\left(n+l+l^{\prime}-1-2 i, i\right)\right) .
$$

Then from the former exact sequence above, an argument similar to Theorem 3.3 shows that $\operatorname{soc}\left(V(l, 0) \otimes P\left(l^{\prime}, 0\right)\right)$ contains a submodule $U$ with $U \cong \operatorname{soc}(V(l, 0) \otimes$ $\left.V\left(l^{\prime}, 0\right)\right) \oplus \operatorname{soc}(P)$. By Proposition $3.1(2)$, we have

$$
\begin{aligned}
U \cong & \left(\oplus_{i=c(t)}^{l^{\prime}-1} V\left(l+l^{\prime}-1-2 i, i\right)\right) \oplus \operatorname{soc}(P) \\
\cong & \left(\oplus_{i=c(t)}^{t} 2 V\left(l+l^{\prime}-1-2 i, i\right)\right) \oplus\left(\oplus_{t+1 \leqslant i \leqslant l^{\prime}-1} V\left(l+l^{\prime}-1-2 i, i\right)\right) \\
& \oplus\left(\oplus_{i=c\left(l+l^{\prime}-1\right)}^{l-1} 2 V\left(n+l+l^{\prime}-1-2 i, i\right)\right) .
\end{aligned}
$$

Then one can check that $\operatorname{dim} P(U)=\operatorname{dim}\left(V(l, 0) \otimes P\left(l^{\prime}, 0\right)\right)$, and so

$$
\begin{aligned}
V(l, 0) \otimes P\left(l^{\prime}, 0\right) \cong P(U) \cong & \left(\oplus_{i=c(t)}^{t} 2 P\left(l+l^{\prime}-1-2 i, i\right)\right) \\
& \oplus\left(\oplus_{t+1 \leqslant i \leqslant l^{\prime}-1} P\left(l+l^{\prime}-1-2 i, i\right)\right) \\
& \oplus\left(\oplus_{i=c\left(l+l^{\prime}-1\right)}^{l-1} 2 P\left(n+l+l^{\prime}-1-2 i, i\right)\right) .
\end{aligned}
$$

This completes the proof.

\subsection{Tensor product of a simple module with a string module.}

In this subsection, we determine the tensor product $V(l, r) \otimes \Omega^{ \pm m} V\left(l^{\prime}, r^{\prime}\right)$ of a simple module with a string module. By [13, p.438], we have

$$
V(l, r) \otimes \Omega^{ \pm m} V\left(l^{\prime}, r^{\prime}\right) \cong \Omega^{ \pm m}\left(V(l, r) \otimes V\left(l^{\prime}, r^{\prime}\right)\right) \oplus P
$$

for some projective module $P$. Moreover, the first summand on the right side of the above isomorphism can be easily determined by Proposition 3.1. But, the projective summand $P$ is not given there. We will use the decomposition of the tensor products of $V(l, r)$ with some composition factors of $\Omega^{ \pm m} V\left(l^{\prime}, r^{\prime}\right)$ to find some projective summands of $V(l, r) \otimes \Omega^{ \pm m} V\left(l^{\prime}, r^{\prime}\right)$, and then compare the dimensions of these modules to determine the projective module $P$. Note that $\Omega^{ \pm m} P=0$ for any $m>0$ and projective module $P$.

Proposition 3.6. Let $1 \leqslant l, l^{\prime}<n$ and $r, r^{\prime} \in \mathbb{Z}_{n}$. Assume that $l+l^{\prime} \leqslant n$. Let $l_{1}=\min \left\{l, l^{\prime}\right\}$. Then for all $m \geqslant 0$, we have

$$
\begin{aligned}
& V(l, r) \otimes \Omega^{ \pm m} V\left(l^{\prime}, r^{\prime}\right) \\
\cong & \left(\oplus_{i=0}^{l_{1}-1} \Omega^{ \pm m} V\left(l+l^{\prime}-1-2 i, r+r^{\prime}+i\right)\right) \\
& \oplus\left(\oplus_{c\left(l+l^{\prime}-1\right) \leqslant i \leqslant l-1}\left(m+\frac{1-(-1)^{m}}{2}\right) P\left(n+l+l^{\prime}-1-2 i, r+r^{\prime}+i\right)\right) .
\end{aligned}
$$

In particular, $V(1, r) \otimes \Omega^{ \pm m} V\left(l^{\prime}, r^{\prime}\right) \cong \Omega^{ \pm m} V\left(l^{\prime}, r+r^{\prime}\right)$.

Proof. As stated above, we have $V(l, r) \otimes \Omega^{ \pm m} V\left(l^{\prime}, r^{\prime}\right) \cong \Omega^{ \pm m}\left(V(l, r) \otimes V\left(l^{\prime}, r^{\prime}\right)\right) \oplus P$ for some projective module $P$. Then by Proposition 3.1(1), we have

$$
\begin{aligned}
\Omega^{ \pm m}\left(V(l, r) \otimes V\left(l^{\prime}, r^{\prime}\right)\right) & \cong \Omega^{ \pm m}\left(\oplus_{i=0}^{l_{1}-1} V\left(l+l^{\prime}-1-2 i, r+r^{\prime}+i\right)\right) \\
& \cong \oplus_{i=0}^{l_{1}-1} \Omega^{ \pm m} V\left(l+l^{\prime}-1-2 i, r+r^{\prime}+i\right) .
\end{aligned}
$$

Hence

$$
V(l, r) \otimes \Omega^{ \pm m} V\left(l^{\prime}, r^{\prime}\right) \cong\left(\oplus_{i=0}^{l_{1}-1} \Omega^{ \pm m} V\left(l+l^{\prime}-1-2 i, r+r^{\prime}+i\right)\right) \oplus P .
$$


If $l \leqslant l^{\prime}$, then a straightforward computation shows that $\operatorname{dim}\left(V(l, r) \otimes \Omega^{ \pm m} V\left(l^{\prime}, r^{\prime}\right)\right)=$ $\operatorname{dim}\left(\oplus_{i=0}^{l-1} \Omega^{ \pm m} V\left(l+l^{\prime}-1-2 i, r+r^{\prime}+i\right)\right)$, which implies $\operatorname{dim}(P)=0$, and so $P=0$. Thus, the desired decomposition follows for $l \leqslant l^{\prime}$.

Now suppose that $l^{\prime}<l$. We may assume that $m$ is odd since the proof is similar for $m$ being even. Then we have two exact sequences

$$
\begin{gathered}
V(l, r) \otimes \Omega^{m} V\left(l^{\prime}, r^{\prime}\right) \rightarrow(m+1) V(l, r) \otimes V\left(n-l^{\prime}, r^{\prime}+l^{\prime}\right) \rightarrow 0, \\
0 \rightarrow(m+1) V(l, r) \otimes V\left(n-l^{\prime}, r^{\prime}+l^{\prime}\right) \rightarrow V(l, r) \otimes \Omega^{-m} V\left(l^{\prime}, r^{\prime}\right) .
\end{gathered}
$$

Note that $l \leqslant n-l^{\prime}$ and $l+n-l^{\prime}-(n+1)=l-l^{\prime}-1 \geqslant 0$. By Proposition 3.1 (2), the projective module $\oplus_{i=c\left(l-l^{\prime}-1\right)}^{l-l^{\prime}-1}(m+1) P\left(l+n-l^{\prime}-1-2 i, r+r^{\prime}+l^{\prime}+i\right)$ is a summand of $(m+1) V(l, 0) \otimes V\left(n-l^{\prime}, l^{\prime}\right)$, and so it is a summand of $V(l, r) \otimes \Omega^{ \pm m} V\left(l^{\prime}, r^{\prime}\right)$. Then by Krull-Schmidt Theorem, we have

$$
\begin{aligned}
& V(l, r) \otimes \Omega^{ \pm m} V\left(l^{\prime}, r^{\prime}\right) \\
\cong & \left(\oplus_{i=0}^{l^{\prime}-1} \Omega^{ \pm m} V\left(l+l^{\prime}-1-2 i, r+r^{\prime}+i\right)\right) \\
& \oplus\left(\oplus_{i=c\left(l-l^{\prime}-1\right)}^{l-l^{\prime}-1}(m+1) P\left(l+n-l^{\prime}-1-2 i, r+r^{\prime}+l^{\prime}+i\right)\right) \oplus Q \\
\cong & \left(\oplus_{i=0}^{l^{\prime}-1} \Omega^{ \pm m} V\left(l+l^{\prime}-1-2 i, r+r^{\prime}+i\right)\right) \\
& \oplus\left(\oplus_{i=c\left(l+l^{\prime}-1\right)}^{l-1}(m+1) P\left(n+l+l^{\prime}-1-2 i, r+r^{\prime}+i\right)\right) \oplus Q,
\end{aligned}
$$

for some projective module $Q$. By a straightforward computation, one finds that $\operatorname{dim} Q=0$, and so $Q=0$. This completes the proof.

Proposition 3.7. Let $1 \leqslant l, l^{\prime} \leqslant n$ with $l^{\prime} \neq n$ and $r, r^{\prime} \in \mathbb{Z}_{n}$. Assume that $t=l+l^{\prime}-(n+1) \geqslant 0$. Let $l_{1}=\min \left\{l, l^{\prime}\right\}$. Then for all $m \geqslant 1$, we have

$$
\begin{aligned}
& V(l, r) \otimes \Omega^{ \pm m} V\left(l^{\prime}, r^{\prime}\right) \\
\cong \quad & \left(\oplus_{t+1 \leqslant i \leqslant l_{1}-1} \Omega^{ \pm m} V\left(l+l^{\prime}-1-2 i, r+r^{\prime}+i\right)\right) \\
& \oplus\left(\oplus_{i=c(t)}^{t}\left(m+\frac{1+(-1)^{m}}{2}\right) P\left(l+l^{\prime}-1-2 i, r+r^{\prime}+i\right)\right) \\
& \oplus\left(\oplus_{c\left(l+l^{\prime}-1\right) \leqslant i \leqslant l-1}\left(m+\frac{1-(-1)^{m}}{2}\right) P\left(n+l+l^{\prime}-1-2 i, r+r^{\prime}+i\right)\right) .
\end{aligned}
$$

Proof. By Proposition 3.1(1), Corollary 3.4 and Proposition 3.6. we only need to consider the case of $r=r^{\prime}=0$. Now by Proposition 3.1 (2), we have

$$
\begin{aligned}
V(l, 0) \otimes \Omega^{ \pm m} V\left(l^{\prime}, 0\right) & \cong \Omega^{ \pm m}\left(V(l, 0) \otimes V\left(l^{\prime}, 0\right)\right) \oplus P \\
& \cong\left(\oplus_{t+1 \leqslant i \leqslant l_{1}-1} \Omega^{ \pm m} V\left(l+l^{\prime}-1-2 i, i\right)\right) \oplus P
\end{aligned}
$$

for some projective module $P$. We assume that $m$ is odd since the proof is similar for $m$ being even. Then we have two exact sequences

$$
\begin{aligned}
0 \rightarrow m V(l, 0) \otimes V\left(l^{\prime}, 0\right) & \rightarrow V(l, 0) \otimes \Omega^{m} V\left(l^{\prime}, 0\right) \\
& \rightarrow(m+1) V(l, 0) \otimes V\left(n-l^{\prime}, l^{\prime}\right) \rightarrow 0, \\
0 \rightarrow(m+1) V(l, 0) \otimes V\left(n-l^{\prime}, l^{\prime}\right) & \rightarrow V(l, 0) \otimes \Omega^{-m} V\left(l^{\prime}, 0\right) \\
& \rightarrow m V(l, 0) \otimes V\left(l^{\prime}, 0\right) \rightarrow 0 .
\end{aligned}
$$

By Proposition $3.1(2)$, the projective module $\oplus_{i=c(t)}^{t} m P\left(l+l^{\prime}-1-2 i, i\right)$ is isomorphic to a summand of $m V(l, 0) \otimes V\left(l^{\prime}, 0\right)$. If $l^{\prime}<l$, then $l+n-l^{\prime}-(n+1)=$ 
$l-l^{\prime}-1 \geqslant 0$ and $n-l^{\prime}<l$ by $l+l^{\prime} \geqslant n+1$. Again by Proposition 3.1(2), $(m+1) V(l, 0) \otimes V\left(n-l^{\prime}, l^{\prime}\right)$ contains a summand isomorphic to

$\oplus_{i=c\left(l-l^{\prime}-1\right)}^{l-l^{\prime}-1}(m+1) P\left(l+n-l^{\prime}-1-2 i, l^{\prime}+i\right) \cong \oplus_{i=c\left(l+l^{\prime}-1\right)}^{l-1}(m+1) P\left(n+l+l^{\prime}-1-2 i, i\right)$ in this case. Thus, $V(l, 0) \otimes \Omega^{ \pm m} V\left(l^{\prime}, 0\right)$ contains a summand isomorphic to

$$
\left(\oplus_{i=c(t)}^{t} m P\left(l+l^{\prime}-1-2 i, i\right)\right) \oplus\left(\oplus_{c\left(l+l^{\prime}-1\right) \leqslant i \leqslant l-1}(m+1) P\left(n+l+l^{\prime}-1-2 i, i\right)\right)
$$

in any case. Then it follows from Krull-Schmidt Theorem that

$$
\begin{aligned}
& V(l, 0) \otimes \Omega^{ \pm m} V\left(l^{\prime}, 0\right) \\
\cong & \left(\oplus_{t+1 \leqslant i \leqslant l_{1}-1} \Omega^{ \pm m} V\left(l+l^{\prime}-1-2 i, i\right)\right) \\
& \oplus\left(\oplus_{i=c(t)}^{t} m P\left(l+l^{\prime}-1-2 i, r+r^{\prime}+i\right)\right) \\
& \oplus\left(\oplus_{c\left(l+l^{\prime}-1\right) \leqslant i \leqslant l-1}(m+1) P\left(n+l+l^{\prime}-1-2 i, i\right)\right) \oplus Q
\end{aligned}
$$

for some projective module $Q$. Then by a tedious but standard computation, one gets that $\operatorname{dim} Q=0$, and so $Q=0$. This completes the proof.

\subsection{Tensor product of a simple module with a band module.}

In this subsection, we investigate the tensor product $M=V(l, r) \otimes M_{s}\left(l^{\prime}, r^{\prime}, \eta\right)$ of a simple module with a band module. Erdmann, Green, Snashall and Taillefer in 13 showed that any non-projective indecomposable summand of $M$ is a band module. They described the module on an example with $s=1$ for the special case $n=d=6$, but the decomposition for general case is not given there. By tensoring with $V(2,0)$, we will determine $M$ by the induction on $l$. For $l=1$ and $l=2$, we determine $M$ by using some standard basis and the duality $(-)^{*}$. For the induction step, we use the following isomorphism (see Proposition 3.1)

$V(2,0) \otimes V(l, r) \otimes M_{s}\left(l^{\prime}, r^{\prime}, \eta\right) \cong V(l+1, r) \otimes M_{s}\left(l^{\prime}, r^{\prime}, \eta\right) \oplus V(l-1, r+1) \otimes M_{s}\left(l^{\prime}, r^{\prime}, \eta\right)$.

If the decompositions of $V(l, r) \otimes M_{s}\left(l^{\prime}, r^{\prime}, \eta\right)$ and $V(l-1, r+1) \otimes M_{s}\left(l^{\prime}, r^{\prime}, \eta\right)$ are known, then the decomposition of the module on the left side is known, which yields the decomposition of $V(l+1, r) \otimes M_{s}\left(l^{\prime}, r^{\prime}, \eta\right)$. Consequently, one gets the decomposition of $M$ for all $1 \leqslant l \leqslant n$.

Lemma 3.8. Let $1 \leqslant l<n, r, r^{\prime} \in \mathbb{Z}_{n}$ and $\eta \in \mathbb{P}^{1}(k)$. Then for all $s \geqslant 1$, $V(1, r) \otimes M_{s}\left(l^{\prime}, r^{\prime}, \eta\right) \cong M_{s}\left(l, r+r^{\prime}, \eta\right)$.

Proof. It is similar to [8, Lemma 3.2 and Proposition 3.4].

For a module $M$, let $M_{(r)}=\left\{m \in M \mid b m=q^{r} m\right\}, r \in \mathbb{Z}_{n}$. Then it follows from [5], Lemma 2.1] that $M=M_{(0)} \oplus M_{(1)} \oplus \cdots \oplus M_{(n-1)}$ as vector spaces and $c M_{(r)} \subseteq M_{(r)}$ for all $r \in \mathbb{Z}_{n}$. If $f: M \rightarrow N$ is a module map, then $f\left(M_{(r)}\right) \subseteq N_{(r)}$ for any $r \in \mathbb{Z}_{n}$.

Lemma 3.9. Let $1 \leqslant l<n, r \in \mathbb{Z}_{n}$ and $s \geqslant 1$. Then there is a basis $\left\{v_{i, j} \mid 1 \leqslant i \leqslant\right.$ $n, 1 \leqslant j \leqslant s\}$ in $M_{s}(l, r, \infty)$ such that

$$
a v_{i, j}=\left\{\begin{array}{ll}
v_{i+1, j-1}, & i=n-l, \\
0, & i=n, \\
v_{i+1, j}, & \text { otherwise }
\end{array} \quad b v_{i, j}=q^{r+l+i-1} v_{i, j},\right.
$$




$$
d v_{i, j}=\left\{\begin{array}{ll}
v_{n, j}, & i=1, \\
\alpha_{i-1}(n-l) v_{i-1, j}, & 1<i \leqslant n-l, \\
0, & i=n-l+1, \\
\alpha_{i-n+l-1}(l) v_{i-1, j}, & n-l+1<i \leqslant n,
\end{array} \quad c v_{i, j}=q^{i-r} v_{i, j},\right.
$$

where $1 \leqslant i \leqslant n, 1 \leqslant j \leqslant s$ and $v_{n-l+1,0}=0$.

Proof. We prove the lemma by the induction on $s$. For $s=1$, it follows from Section 2, Now let $s \geqslant 2$ and $M=M_{s}(l, r, \infty)$. Then by [7, Theorem 3.10(2)], $M$ contains a unique submodule $N$ of $(s-1, s-1)$-type. Moreover, $N \cong M_{s-1}(l, r, \infty)$ and $M / N \cong M_{1}(l, r, \infty)$. By the induction hypothesis, $N$ contains a basis $\left\{v_{i, j} \mid 1 \leqslant\right.$ $i \leqslant n, 1 \leqslant j \leqslant s-1\}$ as stated in the lemma. Define a subspace $L$ of $N$ by $L=\operatorname{span}\left\{v_{i, j} \mid 1 \leqslant i \leqslant n, 1 \leqslant j \leqslant s-2\right\}$ for $s>2$, and $L=0$ for $s=2$. Then $L$ is obviously a submodule of $N$, and $L \cong M_{s-2}(l, r, \infty)$ for $s>2$ by the induction hypothesis. It follows from [7, Theorem 3.10(2)] that $M / L \cong M_{2}(l, r, \infty)$. Since $M / N \cong M_{1}(l, r, \infty), M / N$ contains a standard basis $\left\{x_{1}, x_{2}, \cdots, x_{n}\right\}$ as stated in Section 2. Let $\pi: M \rightarrow M / N$ be the canonical epimorphism. Since $x_{1} \in(M / N)_{(r+l)}$ and $x_{n-l+1} \in(M / N)_{(r)}, x_{1}=\pi\left(u_{1}\right)$ and $x_{n-l+1}=\pi\left(u_{n-l+1}\right)$ for some $u_{1} \in M_{(r+l)}$ and $u_{n-l+1} \in M_{(r)}$. Obviously, $u_{1} \notin N$ and $u_{n-l+1} \notin N$. By [5, Lemma 2.2], we have that $a^{l-1} M_{(r)} \subseteq M_{(r+l-1)}$ and $d M_{(r+l)} \subseteq M_{(r+l-1)}$. From $d x_{1}=x_{n}$, one gets $\pi\left(d u_{1}\right)=\pi\left(a^{l-1} u_{n-l+1}\right)$. Hence $d u_{1}-a^{l-1} u_{n-l+1} \in N \cap$ $M_{(r+l-1)}=N_{(r+l-1)}$, and so $d u_{1}=a^{l-1} u_{n-l+1}+x$ for some $x \in N_{(r+l-1)}$. By the action of $a$ on the basis of $N$ described above, one can see that $a^{l-1} N_{(r)}=N_{(r+l-1)}$. Therefore, there is an element $y \in N_{(r)}$ such that $x=a^{l-1} y$, and consequently, $d u_{1}=a^{l-1}\left(u_{n-l+1}+y\right)$. By replacing $u_{n-l+1}$ with $u_{n-l+1}+y$, we may assume that $x=0$, i.e., $d u_{1}=a^{l-1} u_{n-l+1}$. From $a x_{n-l}=0$ and $a x_{i}=x_{i+1}$ for $1 \leqslant i<n-l$, one gets $\pi\left(a^{n-l} u_{1}\right)=a^{n-l} x_{1}=0$. Hence $a^{n-l} u_{1} \in N \cap M_{(r)}=N_{(r)}$.

Now let $u_{i} \in M, 1 \leqslant i \leqslant n$, be defined by $u_{i}=a^{i-1} u_{1}$ for $1 \leqslant i \leqslant n-l$, and $u_{i}=a^{i-n+l-1} u_{n-l+1}$ for $n-l+1 \leqslant i \leqslant n$. Then $x_{i}=\pi\left(u_{i}\right)$ for all $1 \leqslant i \leqslant n$. By the discussion for $M_{s}(l, r, \infty)$ in Section 2, one knows that $d u_{n-l+1}=0$. Since $\left\{v_{n-l+1, j} \mid 1 \leqslant j \leqslant s-1\right\}$ is a basis of $N_{(r)}$, we have $a^{n-l} u_{1}=\sum_{j=1}^{s-1} \alpha_{j} v_{n-l+1, j}$ for some $\alpha_{1}, \alpha_{2}, \cdots, \alpha_{s-1} \in k$. If $\alpha_{s-1}=0$ then $a^{n-l} u_{1} \in L$. In this case, $\left\{\overline{v_{i, s-1}}, \overline{u_{i}} \mid 1 \leqslant i \leqslant n\right\}$ is a basis of $M / L$, where $\bar{v}$ denotes the image of $v \in M$ under the canonical epimorphism $M \rightarrow M / L$. Obviously, $\operatorname{span}\left\{\overline{v_{i, s-1}} \mid 1 \leqslant i \leqslant n\right\}$ is a submodules of $M / L$. By the discussion for $M_{s}(l, r, \infty)$ in Section 2 together with $d u_{1}=a^{l-1} u_{n-l+1}$ and $d u_{n-l+1}=0$, it is straightforward to check that $\operatorname{span}\left\{\overline{u_{i}} \mid 1 \leqslant\right.$ $i \leqslant n\}$ is also a submodules of $M / L$. Moreover, $M / L=\operatorname{span}\left\{\overline{v_{i, s-1}} \mid 1 \leqslant i \leqslant\right.$ $n\} \oplus \operatorname{span}\left\{\overline{u_{i}} \mid 1 \leqslant i \leqslant n\right\}$. This is impossible since $M / L \cong M_{2}(l, r, \infty)$ is indecomposable. Hence $\alpha_{s-1} \neq 0$. Now let

$$
v_{i, s}=\alpha_{s-1}^{-1}\left(u_{i}-\sum_{1 \leqslant j \leqslant s-2} \alpha_{j} v_{i, j+1}\right), 1 \leqslant i \leqslant n,
$$

where we regard $\sum_{1 \leqslant j \leqslant s-2} \alpha_{j} v_{i, j+1}=0$ for $s=2$. Then $v_{i, s} \in M_{(r+l+i-1)} \backslash N$. Hence $\left\{v_{i, j} \mid 1 \leqslant i \leqslant n, 1 \leqslant j \leqslant s\right\}$ is a basis of $M$. Obviously, $c v_{i, s}=q^{i-r} v_{i, s}$ for all $1 \leqslant i \leqslant n, a v_{n, s}=0$ and $d v_{n-l+1, s}=0$. By [5. Eq.(2.4)] and $a u_{n}=0$, one can check that $d u_{i}=\alpha_{i-1}(n-l) u_{i-1}$ for $1<i \leqslant n-l$. Then a straightforward verification shows that $\left\{v_{i, j} \mid 1 \leqslant i \leqslant n, 1 \leqslant j \leqslant s\right\}$ is a desired basis of $M$. 
Lemma 3.10. Let $1 \leqslant l<n, r \in \mathbb{Z}_{n}, \eta \in k$ and $s \geqslant 1$. Then there is a basis $\left\{v_{i, j} \mid 1 \leqslant i \leqslant n, 1 \leqslant j \leqslant s\right\}$ in $M_{s}(l, r, \eta)$ such that

$$
\begin{gathered}
a v_{i, j}= \begin{cases}v_{i+1, j}, & 1 \leqslant i<n, \quad b v_{i, j}=q^{r+l+i-1} v_{i, j}, \\
0, & i=n,\end{cases} \\
d v_{i, j}=\left\{\begin{array}{ll}
v_{n, j-1}+\eta q^{l} v_{n, j}, & i=1, \\
\alpha_{i-1}(n-l) v_{i-1, j}, & 1<i \leqslant n-l, \\
0, & i=n-l+1, \\
\alpha_{i-n+l-1}(l) v_{i-1, j}, & n-l+1<i \leqslant n,
\end{array} \quad c v_{i, j}=q^{i-r} v_{i, j},\right.
\end{gathered}
$$

where $1 \leqslant i \leqslant n, 1 \leqslant j \leqslant s$ and $v_{n, 0}=0$.

Proof. It is similar to Lemma 3.9 ,

Lemma 3.11. Let $r, r^{\prime} \in \mathbb{Z}_{n}, \eta \in \mathbb{P}^{1}(k)$ and $s \geqslant 1$. Then

$$
V(2, r) \otimes M_{s}\left(1, r^{\prime}, \eta\right) \cong M_{s}\left(2, r+r^{\prime}, \eta q^{-1}(2)_{q}\right) \oplus s V\left(n, r+r^{\prime}+1\right) .
$$

Proof. By Proposition 3.1(1) and Lemma 3.8, we may assume that $r=r^{\prime}=0$. We only consider the case of $\eta \in k$ since the proof is similar for $\eta=\infty$. Assume $\eta \in k$ and let $M=V(2,0) \otimes M_{s}(1,0, \eta)$. By the discussion in Section 2 there is a standard basis $\left\{v_{1}, v_{2}\right\}$ in $V(2,0)$ such that

$$
\begin{array}{llll}
a v_{1}=v_{2}, & b v_{1}=v_{1}, & c v_{1}=q^{-1} v_{1}, & d v_{1}=0, \\
a v_{2}=0, & b v_{2}=q v_{2}, & c v_{2}=v_{2}, & d v_{2}=\alpha_{1}(2) v_{1} .
\end{array}
$$

By Lemma 3.10, there is a standard basis $\left\{v_{i, j} \mid 1 \leqslant i \leqslant n, 1 \leqslant j \leqslant s\right\}$ in $M_{s}(1,0, \eta)$ such that for all $1 \leqslant i \leqslant n$ and $1 \leqslant j \leqslant s$,

$$
\begin{aligned}
& a v_{i, j}=\left\{\begin{array}{ll}
v_{i+1, j}, & 1 \leqslant i<n, \\
0, & i=n,
\end{array} \quad b v_{i, j}=q^{i} v_{i, j},\right. \\
& d v_{i, j}= \begin{cases}v_{n, j-1}+\eta q v_{n, j}, & i=1, \\
\alpha_{i-1}(n-1) v_{i-1, j}, & 1<i \leqslant n-1, \\
0, & i=n,\end{cases}
\end{aligned}
$$

where $v_{n, 0}=0$. Hence $\left\{v_{1} \otimes v_{i, j}, v_{2} \otimes v_{i, j} \mid 1 \leqslant i \leqslant n, 1 \leqslant j \leqslant s\right\}$ is a basis of $M$.

For any $1 \leqslant i \leqslant n$ and $1 \leqslant j \leqslant s$, define $u_{i, j} \in M$ by $u_{1, j}=\left((2)_{q}\right)^{s-j}\left(v_{1} \otimes v_{2, j}+\right.$ $\left.(2)_{q} v_{2} \otimes v_{1, j}\right)$ and $u_{i, j}=a^{i-1} u_{1, j}$ for $i>1$. Then by Lemma 3.10, a straightforward verification shows that $N:=\operatorname{span}\left\{u_{i, j} \mid 1 \leqslant i \leqslant n, 1 \leqslant j \leqslant s\right\}$ is a submodule of $M$ and $N \cong M_{s}\left(2,0, \eta q^{-1}(2)_{q}\right)$.

Since $M_{s}(1,0, \eta) / \operatorname{soc}\left(M_{s}(1,0, \eta)\right) \cong s V(n-1,1)$, there is an epimorphism from $M$ to $s(V(2,0) \otimes V(n-1,1))$. By Proposition $3.1(2), V(n, 1)$ is a projective summand of $V(2,0) \otimes V(n-1,1)$. It follows that $M$ contains a submodule $U$ isomorphic to $s V(n, 1)$. Obviously, $N \cap U=0$. Therefore, $M=N \oplus U \cong M_{s}\left(2,0, \eta q^{-1}(2)_{q}\right) \oplus$ $s V(n, 1)$ by $\operatorname{dim}(N \oplus U)=\operatorname{dim}(M)$.

Lemma 3.12. Let $1 \leqslant l \leqslant n$ and $r \in \mathbb{Z}_{n}$. Then $V(l, r)^{*} \cong V(l, 1-l-r)$ and $P(l, r)^{*} \cong P(l, 1-l-r)$. If $1 \leqslant l<n$, then $\left(\Omega^{m} V(l, r)\right)^{*} \cong \Omega^{-m} V(l, 1-l-r)$ and $\left(\Omega^{-m} V(l, r)\right)^{*} \cong \Omega^{m} V(l, 1-l-r)$ for all $m \geqslant 1$. 
Proof. The first isomorphism is due to [1, Theorem 4.3], and the rest follow from an argument similar to [8, Lemma 3.16].

Lemma 3.13. Let $1 \leqslant l<n, r \in \mathbb{Z}_{n}, \eta \in \mathbb{P}^{1}(k)$ and $s \geqslant 1$. Then

$$
M_{s}(l, r, \eta)^{*} \cong M_{s}\left(n-l, 1-r,-\eta q^{l}\right) \text {. }
$$

Proof. At first, by an argument similar to [1, Theorem 4.3], one can check that $M_{1}(l, r, \eta)^{*} \cong M_{1}\left(n-l, 1-r,-\eta q^{l}\right)$ for $\eta=\infty$ and $\eta \in k$, respectively.

Now assume $s>1$. Then $M_{s}(l, r, \eta)^{*}$ is indecomposable. By the structure of $M_{s}(l, r, \eta)$, we have an exact sequence $0 \rightarrow s V(l, r) \rightarrow M_{s}(l, r, \eta) \rightarrow s V(n-l, r+$ $l) \rightarrow 0$. Applying the duality $(-)^{*}$ to the above exact sequence and using Lemma 3.12, one gets another exact sequence

$$
0 \rightarrow s V(n-l, 1-r) \rightarrow M_{s}(l, r, \eta)^{*} \rightarrow s V(l, 1-r-l) \rightarrow 0 .
$$

By the classification of indecomposable modules stated in Section 2, one knows that $M_{s}(l, r, \eta)^{*} \cong M_{s}(n-l, 1-r, \alpha)$ for some $\alpha \in \mathbb{P}^{1}(k)$. On the other hand, there is an epimorphism $M_{s}(l, r, \eta) \rightarrow M_{1}(l, r, \eta)$ by [7, Theorem 3.10(2)]. Then by applying the duality $(-)^{*}$, one gets a monomorphism $M_{1}\left(n-l, 1-r,-\eta q^{l}\right) \rightarrow$ $M_{s}(n-l, 1-r, \alpha)$. Again by [7, Theorem 3.10(2)], $M_{s}(n-l, 1-r, \alpha)$ contains a unique submodule of $(1,1)$-type, which is isomorphic to $M_{1}(n-l, 1-r, \alpha)$. Hence $M_{1}\left(n-l, 1-r,-\eta q^{l}\right) \cong M_{1}(n-l, 1-r, \alpha)$, which implies $\alpha=-\eta q^{l}$ by [7, Theorem $3.10(4)]$. It follows that $M_{s}(l, r, \eta)^{*} \cong M_{s}\left(n-l, 1-r,-\eta q^{l}\right)$.

Corollary 3.14. Let $r, r^{\prime} \in \mathbb{Z}_{n}, \eta \in \mathbb{P}^{1}(k)$ and $s \geqslant 1$. Then

$$
V(2, r) \otimes M_{s}\left(n-1, r^{\prime}, \eta\right) \cong M_{s}\left(n-2, r+r^{\prime}+1, \eta(2)_{q}\right) \oplus s V\left(n, r+r^{\prime}\right)
$$

Proof. It is enough to show the corollary for $r=r^{\prime}=0$. By Lemma 3.11, we have an isomorphism $V(2,-1) \otimes M_{s}\left(1,1,-\eta q^{-1}\right) \cong M_{s}\left(2,0,-\eta q^{-2}(2)_{q}\right) \oplus s V(n, 1)$. Then by applying the duality $(-)^{*}$ to the isomorphism, it follows from Lemmas 3.12 and 3.13 that $V(2,0) \otimes M_{s}(n-1,0, \eta) \cong M_{s}\left(n-2,1, \eta(2)_{q}\right) \oplus s V(n, 0)$.

Lemma 3.15. Let $1<l^{\prime}<n-1, r, r^{\prime} \in \mathbb{Z}_{n}, \eta \in \mathbb{P}^{1}(k)$ and $s \geqslant 1$. Then

$$
\begin{aligned}
& V(2, r) \otimes M_{s}\left(l^{\prime}, r^{\prime}, \eta\right) \\
\cong & M_{s}\left(l^{\prime}+1, r+r^{\prime}, \eta q^{-1} \frac{\left(l^{\prime}+1\right)_{q}}{\left(l^{\prime}\right)_{q}}\right) \oplus M_{s}\left(l^{\prime}-1, r+r^{\prime}+1, \eta q \frac{\left(l^{\prime}-1\right)_{q}}{\left(l^{\prime}\right)_{q}}\right) .
\end{aligned}
$$

Proof. It is enough to show the lemma for $r=r^{\prime}=0$. We only prove the lemma for $\eta \in k$ since the proof is similar for $\eta=\infty$.

Assume $\eta \in k$ and let $M=V(2,0) \otimes M_{s}\left(l^{\prime}, 0, \eta\right)$. Let $\left\{v_{1}, v_{2}\right\}$ be the standard basis of $V(2,0)$ as stated in the proof of Lemma 3.11 and let $\left\{v_{i, j} \mid 1 \leqslant i \leqslant n, 1 \leqslant j \leqslant s\right\}$ be the standard basis of $M_{s}\left(l^{\prime}, 0, \eta\right)$ as given in Lemma 3.10. Then $M$ has a $k$-basis $\left\{v_{1} \otimes v_{i, j}, v_{2} \otimes v_{i, j} \mid 1 \leqslant i \leqslant n, 1 \leqslant j \leqslant s\right\}$.

Now let $\beta=\frac{q^{l^{\prime}}}{(1-q)\left(l^{\prime}\right)_{q}}$ and $\gamma=\frac{\left(l^{\prime}\right)_{q}}{\left(l^{\prime}-1\right)_{q}}$. For $1 \leqslant i \leqslant n$ and $1 \leqslant j \leqslant s$, define $u_{i, j} \in M$ by $u_{1, j}=\gamma^{j}\left(v_{1} \otimes v_{1, j}+\eta q^{l^{\prime}} \beta v_{2} \otimes v_{n, j}+\beta v_{2} \otimes v_{n, j-1}\right)$ and $u_{i, j}=a^{i-1} u_{1, j}$ for $1<i \leqslant n$. Then by a standard computation, one can check that $N_{1}:=\operatorname{span}\left\{u_{i, j} \mid 1 \leqslant i \leqslant n, 1 \leqslant\right.$ 
$j \leqslant s\}$ is a submodule of $M$ and $N_{1} \cong M_{s}\left(l^{\prime}-1,1, \eta q \gamma^{-1}\right)=M_{s}\left(l^{\prime}-1,1, \eta q \frac{\left(l^{\prime}-1\right)_{q}}{\left(l^{\prime}\right)_{q}}\right)$ by Lemma 3.10 .

Next, let $\theta=\frac{\left(l^{\prime}\right)_{q}}{\left(l^{\prime}+1\right)_{q}}$. For $1 \leqslant i \leqslant n$ and $1 \leqslant j \leqslant s$, define $w_{i, j} \in M$ by $w_{1, j}=$ $\theta^{j}\left(v_{1} \otimes v_{2, j}+\left(l^{\prime}+1\right)_{q} v_{2} \otimes v_{1, j}\right)$ and $w_{i, j}=a^{i-1} w_{1, j}$ for $1<i \leqslant n$. Then a straightforward verification shows that $N_{2}:=\operatorname{span}\left\{w_{i, j} \mid 1 \leqslant i \leqslant n, 1 \leqslant j \leqslant s\right\}$ is a submodule of $M$ and $N_{2} \cong M_{s}\left(l^{\prime}+1,0, \eta q^{-1} \theta^{-1}\right)=M_{s}\left(l^{\prime}+1,0, \eta q^{-1} \frac{\left(l^{\prime}+1\right)_{q}}{\left(l^{\prime}\right)_{q}}\right)$.

Finally, since $\operatorname{soc}\left(N_{1}\right) \cong s V\left(l^{\prime}-1,1\right)$ and $\operatorname{soc}\left(N_{2}\right) \cong s V\left(l^{\prime}+1,0\right)$, the sum $N_{1}+N_{2}$ is direct. Then it follows from $\operatorname{dim}(M)=\operatorname{dim}\left(N_{1} \oplus N_{2}\right)$ that

$$
M=N_{1} \oplus N_{2} \cong M_{s}\left(l^{\prime}-1,1, \eta q \frac{\left(l^{\prime}-1\right)_{q}}{\left(l^{\prime}\right)_{q}}\right) \oplus M_{s}\left(l^{\prime}+1,0, \eta q^{-1} \frac{\left(l^{\prime}+1\right)_{q}}{\left(l^{\prime}\right)_{q}}\right) .
$$

Theorem 3.16. Let $1 \leqslant l, l^{\prime}<n, r, r^{\prime} \in \mathbb{Z}_{n}, \eta \in \mathbb{P}^{1}(k)$ and $s \geqslant 1$. Assume that $l+l^{\prime} \leqslant n$ and let $l_{1}=\min \left\{l, l^{\prime}\right\}$. Then

$$
\begin{aligned}
& V(l, r) \otimes M_{s}\left(l^{\prime}, r^{\prime}, \eta\right) \\
\cong & \left(\oplus_{i=0}^{l_{1}-1} M_{s}\left(l+l^{\prime}-1-2 i, r+r^{\prime}+i, \eta q^{2 i-l+1} \frac{\left(l+l^{\prime}-1-2 i\right)_{q}}{\left(l^{\prime}\right)_{q}}\right)\right) \\
& \oplus\left(\oplus_{c\left(l+l^{\prime}-1\right) \leqslant i \leqslant l-1} s P\left(n+l+l^{\prime}-1-2 i, r+r^{\prime}+i\right)\right) .
\end{aligned}
$$

Proof. It is enough to show the proposition for $r=r^{\prime}=0$. We prove it by the induction on $l$. For $l=1$ and $l=2$, it follows from Lemmas 3.8, 3.11 and 3.15. respectively. Now let $l>2$ and assume that the theorem holds for less $l$.

Case 1: $l \leqslant l^{\prime}$. In this case, $l-2<l-1<l^{\prime}$. Hence by the induction hypothesis, Lemma 3.15 and Proposition 3.1(1), we have

$$
\begin{aligned}
& V(2,0) \otimes V(l-1,0) \otimes M_{s}\left(l^{\prime}, 0, \eta\right) \\
\cong & \oplus_{i=0}^{l-2} V(2,0) \otimes M_{s}\left(l+l^{\prime}-2-2 i, i, \eta q^{2 i-l+2} \frac{\left(l^{\prime}+l-2-2 i\right)_{q}}{\left(l^{\prime}\right)_{q}}\right) \\
\cong & \left(\oplus _ { i = 0 } ^ { l - 2 } M _ { s } \left(l+l^{\prime}-1-2 i, i, \eta q^{2 i-l+1} \frac{\left(l^{\prime}+l-1-2 i\right)_{q}}{\left.\left(l^{\prime}\right)_{q}\right)}\right.\right. \\
& \oplus\left(\oplus_{i=0}^{l-2} M_{s}\left(l+l^{\prime}-3-2 i, i+1, \eta q^{2 i-l+3} \frac{\left(l^{\prime}+l-3-2 i\right)_{q}}{\left(l^{\prime}\right)_{q}}\right)\right)
\end{aligned}
$$

and

$$
\begin{aligned}
& V(2,0) \otimes V(l-1,0) \otimes M_{s}\left(l^{\prime}, 0, \eta\right) \\
\cong & V(l, 0) \otimes M_{s}\left(l^{\prime}, 0, \eta\right) \oplus V(l-2,1) \otimes M_{s}\left(l^{\prime}, 0, \eta\right) \\
\cong & V(l, 0) \otimes M_{s}\left(l^{\prime}, 0, \eta\right) \oplus\left(\oplus_{i=0}^{l-3} M_{s}\left(l+l^{\prime}-3-2 i, i+1, \eta q^{2 i-l+3} \frac{\left(l+l^{\prime}-3-2 i\right)_{q}}{\left(l^{\prime}\right)_{q}}\right)\right) .
\end{aligned}
$$

Thus, using Krull-Schmidt Theorem, one gets that

$$
V(l, 0) \otimes M_{s}\left(l^{\prime}, 0, \eta\right) \cong \oplus_{i=0}^{l-1} M_{s}\left(l+l^{\prime}-1-2 i, i, \eta q^{2 i-l+1} \frac{\left(l+l^{\prime}-1-2 i\right)_{q}}{\left(l^{\prime}\right)_{q}}\right) .
$$


Case 2: $l=l^{\prime}+1$. In this case, $l-2<l-1=l^{\prime}$. Hence by the induction hypothesis, and Lemmas 3.11 and 3.15 , we have

$$
\begin{aligned}
& V(2,0) \otimes V(l-1,0) \otimes M_{s}\left(l^{\prime}, 0, \eta\right) \\
\cong & \oplus_{i=0}^{l-2} V(2,0) \otimes M_{s}\left(l+l^{\prime}-2-2 i, i, \eta q^{2 i-l+2} \frac{\left(l^{\prime}+l-2-2 i\right)_{q}}{\left(l^{\prime}\right)_{q}}\right) \\
\cong & V(2,0) \otimes M_{s}\left(1, l^{\prime}-1, \eta q^{l-2} \frac{1}{\left(l^{\prime}\right)_{q}}\right) \\
& \oplus\left(\oplus_{i=0}^{l^{\prime}-2} V(2,0) \otimes M_{s}\left(l+l^{\prime}-2-2 i, i, \eta q^{2 i-l+2} \frac{\left(l^{\prime}+l-2-2 i\right)_{q}}{\left(l^{\prime}\right)_{q}}\right)\right) \\
\cong & M_{s}\left(2, l^{\prime}-1, \eta q^{l-3} \frac{(2)_{q}}{\left(l^{\prime}\right)_{q}}\right) \oplus s V\left(n, l^{\prime}\right) \\
& \oplus\left(\oplus_{i=0}^{l^{\prime}-2} M_{s}\left(l+l^{\prime}-1-2 i, i, \eta q^{2 i-l+1} \frac{\left(l^{\prime}+l-1-2 i\right)_{q}}{\left(l^{\prime}\right)_{q}}\right)\right) \\
& \oplus\left(\oplus_{i=0}^{l^{\prime}-2} M_{s}\left(l+l^{\prime}-3-2 i, i+1, \eta q^{2 i-l+3} \frac{\left(l^{\prime}+l-3-2 i\right)_{q}}{\left(l^{\prime}\right)_{q}}\right)\right) .
\end{aligned}
$$

Then an argument similar to Case 1 shows that

$$
\begin{aligned}
& V(l, 0) \otimes M_{s}\left(l^{\prime}, 0, \eta\right) \\
\cong & \left(\oplus_{i=0}^{l^{\prime}-1} M_{s}\left(l+l^{\prime}-1-2 i, i, \eta q^{2 i-l+1} \frac{\left(l+l^{\prime}-1-2 i\right)_{q}}{\left(l^{\prime}\right)_{q}}\right)\right) \oplus s V\left(n, l^{\prime}\right) .
\end{aligned}
$$

Case 3: $l=l^{\prime}+2$. In this case, $l^{\prime}=l-2<l-1$. Hence by the induction hypothesis (or Case 2), Proposition 3.1(2) and Lemma 3.15, we have

$$
\begin{aligned}
& V(2,0) \otimes V(l-1,0) \otimes M_{s}\left(l^{\prime}, 0, \eta\right) \\
\cong & \left(\oplus_{i=0}^{l^{\prime}-1} V(2,0) \otimes M_{s}\left(l+l^{\prime}-2-2 i, i, \eta q^{2 i-l+2} \frac{\left(l+l^{\prime}-2-2 i\right)_{q}}{\left(l^{\prime}\right)_{q}}\right)\right) \\
& \oplus s V(2,0) \otimes V\left(n, l^{\prime}\right) \\
\cong & \left(\oplus_{i=0}^{l^{\prime}-1} M_{s}\left(l+l^{\prime}-1-2 i, i, \eta q^{2 i-l+1} \frac{\left(l^{\prime}+l-1-2 i\right)_{q}}{\left(l^{\prime}\right)_{q}}\right)\right) \\
& \oplus\left(\oplus_{i=0}^{l^{\prime}-1} M_{s}\left(l+l^{\prime}-3-2 i, i+1, \eta q^{2 i-l+3} \frac{\left(l^{\prime}+l-3-2 i\right)_{q}}{\left(l^{\prime}\right)_{q}}\right)\right) \oplus s P\left(n-1, l^{\prime}+1\right) .
\end{aligned}
$$

Then an argument similar to Case 1 shows that

$$
\begin{aligned}
& V(l, 0) \otimes M_{s}\left(l^{\prime}, 0, \eta\right) \\
\cong & \left(\oplus_{i=0}^{l^{\prime}-1} M_{s}\left(l+l^{\prime}-1-2 i, i, \eta q^{2 i-l+1} \frac{\left(l+l^{\prime}-1-2 i\right)_{q}}{\left(l^{\prime}\right)_{q}}\right)\right) \oplus s P\left(n-1, l^{\prime}+1\right) .
\end{aligned}
$$

Case 4: $l>l^{\prime}+2$. In this case, $l-1>l-2>l^{\prime}$. We assume that $l+l^{\prime}$ is odd since the proof is similar for $l+l^{\prime}$ being even. Then $c\left(l+l^{\prime}-1\right)=c\left(l+l^{\prime}-2\right)$. By 
the induction hypothesis, Lemma 3.15 and Theorems 3.3 and 3.5, we have

$$
\begin{aligned}
& V(2,0) \otimes V(l-1,0) \otimes M_{s}\left(l^{\prime}, 0, \eta\right) \\
\cong & \left(\oplus_{i=0}^{l^{\prime}-1} V(2,0) \otimes M_{s}\left(l+l^{\prime}-2-2 i, i, \eta q^{2 i-l+2} \frac{\left(l+l^{\prime}-2-2 i\right)_{q}}{\left(l^{\prime}\right)_{q}}\right)\right) \\
& \oplus s V(2,0) \otimes P\left(n-1, c\left(l+l^{\prime}-2\right)\right) \\
& \oplus\left(\oplus_{c\left(l+l^{\prime}\right) \leqslant i \leqslant l-2} s V(2,0) \otimes P\left(n+l+l^{\prime}-2-2 i, i\right)\right) \\
\cong & \left(\oplus_{i=0}^{l^{\prime}-1} M_{s}\left(l+l^{\prime}-1-2 i, i, \eta q^{2 i-l+1} \frac{\left(l^{\prime}+l-1-2 i\right)_{q}}{\left(l^{\prime}\right)}\right)\right) \\
& \oplus\left(\oplus_{i=0}^{l^{\prime}-1} M_{s}\left(l+l^{\prime}-3-2 i, i+1, \eta q^{2 i-l+3} \frac{\left(l^{\prime}+l-3-2 i\right)_{q}}{\left(l^{\prime}\right)_{q}}\right)\right) \\
& \oplus 2 s P\left(n, c\left(l+l^{\prime}-1\right)\right) \oplus s P\left(n-2, c\left(l+l^{\prime}+1\right)\right) \\
& \oplus\left(\oplus_{c\left(l+l^{\prime}+1\right) \leqslant i \leqslant l-2} s\left(P\left(n+l+l^{\prime}-1-2 i, i\right) \oplus P\left(n+l+l^{\prime}-3-2 i, i+1\right)\right)\right. \\
\cong & \left(\oplus_{i=0}^{l^{\prime}-1} M_{s}\left(l+l^{\prime}-1-2 i, i, \eta q^{2 i-l+1} \frac{\left(l^{\prime}+l-1-2 i\right)_{q}}{\left(l^{\prime}\right)}\right)\right) \\
& \oplus\left(\oplus_{i=0}^{l^{\prime}-1} M_{s}\left(l+l^{\prime}-3-2 i, i+1, \eta q^{2 i-l+3} \frac{\left(l^{\prime}+l-3-2 i\right)_{q}}{\left(l^{\prime}\right)_{q}}\right)\right) \\
& \oplus\left(\oplus_{i=c}^{l-2}\right) \\
& \oplus\left(\oplus_{i=c\left(l+l^{\prime}-1\right)}^{l-1} s P\left(n+l+l^{\prime}-1-2 i, i\right)\right)
\end{aligned}
$$

Then an argument similar to Case 1 shows that

$$
\begin{aligned}
& V(l, 0) \otimes M_{s}\left(l^{\prime}, 0, \eta\right) \\
\cong & \left(\oplus_{i=0}^{l^{\prime}-1} M_{s}\left(l+l^{\prime}-1-2 i, i, \eta q^{2 i-l+1} \frac{\left(l+l^{\prime}-1-2 i\right)_{q}}{\left(l^{\prime}\right)_{q}}\right)\right) \\
& \oplus\left(\oplus_{i=c\left(l+l^{\prime}-1\right)}^{l-1} s P\left(n+l+l^{\prime}-1-2 i, i\right)\right) .
\end{aligned}
$$

This completes the proof.

Theorem 3.17. Let $1 \leqslant l, l^{\prime} \leqslant n$ with $l^{\prime} \neq n, r, r^{\prime} \in \mathbb{Z}_{n}, s \geqslant 1$ and $\eta \in \mathbb{P}^{1}(k)$. Assume that $t=l+l^{\prime}-(n+1) \geqslant 0$. Let $l_{1}=\min \left\{l, l^{\prime}\right\}$. Then

$$
\begin{aligned}
& V(l, r) \otimes M_{s}\left(l^{\prime}, r^{\prime}, \eta\right) \\
\cong & \left(\oplus_{t+1 \leqslant i \leqslant l_{1}-1} M_{s}\left(l+l^{\prime}-1-2 i, r+r^{\prime}+i, \eta q^{2 i-l+1} \frac{\left(l+l^{\prime}-1-2 i\right)_{q}}{\left(l^{\prime}\right)_{q}}\right)\right) \\
& \oplus\left(\oplus_{i=c(t)}^{t} s P\left(l+l^{\prime}-1-2 i, r+r^{\prime}+i\right)\right) \\
& \oplus\left(\oplus_{c\left(l+l^{\prime}-1\right) \leqslant i \leqslant l-1} s P\left(n+l+l^{\prime}-1-2 i, r+r^{\prime}+i\right)\right) .
\end{aligned}
$$

Proof. It is enough to show the theorem for $r=r^{\prime}=0$. We prove it by the induction on $l$ for the three cases: $t=0, t=1$ and $t \geqslant 2$, respectively. Note that $l^{\prime}=t+n+1-l \geqslant t+1$ by $l \leqslant n$, and $l \geqslant 2$ by $l^{\prime}<n$ and $l+l^{\prime} \geqslant n+1$. If $l=2$, then $l^{\prime}=n-1$. In this case, the desired decomposition follows from Corollary 3.14 Now assume that $l>2$.

Suppose $t=0$. If $l \leqslant l^{\prime}+2$, then the desired decomposition follows from an argument similar to Theorem 3.16. Now let $l>l^{\prime}+2$. Then by Theorem 3.16, we have

$$
\begin{aligned}
V(l-2,1) \otimes M_{s}\left(l^{\prime}, 0, \eta\right) \cong & \left(\oplus_{i=0}^{l^{\prime}-1} M_{s}\left(l+l^{\prime}-3-2 i, i+1, \eta q^{2 i-l+3} \frac{\left(l+l^{\prime}-3-2 i\right)_{q}}{\left(l^{\prime}\right)_{q}}\right)\right) \\
& \oplus\left(\oplus_{i=c\left(l+l^{\prime}-3\right)}^{l-3} s P\left(n+l+l^{\prime}-3-2 i, i+1\right)\right) \\
\cong & \left(\oplus_{i=1}^{l^{\prime}} M_{s}\left(l+l^{\prime}-1-2 i, i, \eta q^{2 i-l+1} \frac{\left(l+l^{\prime}-1-2 i\right)_{q}}{\left(l^{\prime}\right)_{q}}\right)\right) \\
& \oplus\left(\oplus_{i=c\left(l+l^{\prime}-1\right)}^{l-2} s P\left(n+l+l^{\prime}-1-2 i, i\right)\right)
\end{aligned}
$$


and

$$
\begin{aligned}
V(l-1,0) \otimes M_{s}\left(l^{\prime}, 0, \eta\right) \cong & \left(\oplus_{i=0}^{l^{\prime}-1} M_{s}\left(l+l^{\prime}-2-2 i, i, \eta q^{2 i-l+2} \frac{\left(l+l^{\prime}-2-2 i\right)_{q}}{\left(l^{\prime}\right)_{q}}\right)\right) \\
& \oplus\left(\oplus_{i=c\left(l+l^{\prime}-2\right)}^{l-2} s P\left(n+l+l^{\prime}-2-2 i, i\right)\right) .
\end{aligned}
$$

We may assume $l+l^{\prime}$ is odd since the proof is similar when $l+l^{\prime}$ is even. Then $c\left(l+l^{\prime}-2\right)=c\left(l+l^{\prime}-1\right)=\frac{l+l^{\prime}-1}{2}$. Hence by Theorems 3.3 and 3.5. Corollary 3.14 and Lemma 3.15, one can check that

$$
\begin{aligned}
& V(2,0) \otimes V(l-1,0) \otimes M_{s}\left(l^{\prime}, 0, \eta\right) \\
\cong & V(2,0) \otimes M_{s}\left(n-1,0, \eta q^{2-l} \frac{(n-1)_{q}}{\left(l^{\prime}\right)_{q}}\right) \\
& \oplus\left(\oplus_{1 \leqslant i \leqslant l^{\prime}-1} V(2,0) \otimes M_{s}\left(l+l^{\prime}-2-2 i, i, \eta q^{2 i-l+2} \frac{\left(l+l^{\prime}-2-2 i\right)_{q}}{\left(l^{\prime}\right)_{q}}\right)\right) \\
& \oplus s V(2,0) \otimes P\left(n-1, \frac{l+l^{\prime}-1}{2}\right) \\
& \oplus\left(\oplus_{c\left(l+l^{\prime}-1\right)<i \leqslant l-2} s V(2,0) \otimes P\left(n+l+l^{\prime}-2-2 i, i\right)\right) \\
\cong & s V(n, 0) \oplus\left(\oplus_{1 \leqslant i \leqslant l^{\prime}-1} M_{s}\left(l+l^{\prime}-1-2 i, i, \eta q^{2 i-l+1} \frac{\left(l+l^{\prime}-1-2 i\right)_{q}}{\left(l^{\prime}\right)_{q}}\right)\right) \\
& \oplus\left(\oplus_{i=1}^{l^{\prime}} M_{s}\left(l+l^{\prime}-1-2 i, i, \eta q^{2 i-l+1} \frac{\left(l+l^{\prime}-1-2 i\right)_{q}}{\left(l^{\prime}\right)_{q}}\right)\right) \\
& \oplus\left(\oplus_{i=c\left(l+l^{\prime}-1\right)}^{l-2} s P\left(n+l+l^{\prime}-1-2 i, i\right)\right) \\
& \oplus\left(\oplus_{i=c\left(l+l^{\prime}-1\right)}^{l-1} s P\left(n+l+l^{\prime}-1-2 i, i\right)\right) .
\end{aligned}
$$

Thus, it follows from an argument similar to the proof of Theorem 3.16 that

$$
\begin{aligned}
V(l, 0) \otimes M_{s}\left(l^{\prime}, 0, \eta\right) \cong & \left(\oplus_{1 \leqslant i \leqslant l^{\prime}-1} M_{s}\left(l+l^{\prime}-1-2 i, i, \eta q^{2 i-l+1} \frac{\left(l+l^{\prime}-1-2 i\right)_{q}}{\left(l^{\prime}\right)_{q}}\right)\right) \\
& \oplus s V(n, 0) \oplus\left(\oplus_{i=c\left(l+l^{\prime}-1\right)}^{l-1} s P\left(n+l+l^{\prime}-1-2 i, i\right)\right) .
\end{aligned}
$$

For $t=1$, the proof is similar to the case of $t=0$. Now suppose $t \geqslant 2$. If $l \leqslant l^{\prime}+2$, then the desired decomposition follows from an argument similar to Theorem 3.16 Now let $l>l^{\prime}+2$. Then by the induction hypothesis, we have

$$
\begin{aligned}
& V(l-2,1) \otimes M_{s}\left(l^{\prime}, 0, \eta\right) \\
\cong & \left(\oplus_{i=c(t-2)}^{t-2} s P\left(l+l^{\prime}-3-2 i, i+1\right)\right) \\
& \oplus\left(\oplus_{i=t-1}^{l^{\prime}-1} M_{s}\left(l+l^{\prime}-3-2 i, i+1, \eta q^{2 i-l+3} \frac{\left(l+l^{\prime}-3-2 i\right)_{q}}{\left(l^{\prime}\right)_{q}}\right)\right) \\
& \oplus\left(\oplus_{i=c\left(l+l^{\prime}-3\right)}^{l-3} s P\left(n+l+l^{\prime}-3-2 i, i+1\right)\right)
\end{aligned}
$$

and

$$
\begin{aligned}
V(l-1,0) \otimes M_{s}\left(l^{\prime}, 0, \eta\right) \cong & \left(\oplus_{i=c(t-1)}^{t-1} s P\left(l+l^{\prime}-2-2 i, i\right)\right) \\
& \oplus\left(\oplus_{i=t}^{l^{\prime}-1} M_{s}\left(l+l^{\prime}-2-2 i, i, \eta q^{2 i-l+2} \frac{\left(l+l^{\prime}-2-2 i\right)_{q}}{\left(l^{\prime}\right)_{q}}\right)\right) \\
& \oplus\left(\oplus_{i=c\left(l+l^{\prime}-2\right)}^{l-2} s P\left(n+l+l^{\prime}-2-2 i, i\right)\right)
\end{aligned}
$$

In the following, we only consider the case that $t$ and $l+l^{\prime}$ are both odd, since the proofs are similar for the other cases. In this case, $c(t)=c(t-1)+1=\frac{t+1}{2}$ and $c\left(l+l^{\prime}-1\right)=c\left(l+l^{\prime}-2\right)=\frac{l+l^{\prime}-1}{2}$. By Proposition 3.1 (2), Theorems 3.3, 3.5 and 
Lemma 3.15, a straightforward computation shows that

$$
\begin{aligned}
& V(2,0) \otimes V(l-1,0) \otimes M_{s}\left(l^{\prime}, 0, \eta\right) \\
\cong & \left(\oplus_{i=c(t)}^{t-1} s P\left(l+l^{\prime}-1-2 i, i\right)\right) \oplus\left(\oplus_{i=c(t)}^{t} s P\left(l+l^{\prime}-1-2 i, i\right)\right) \\
& \oplus\left(\oplus_{i=t}^{l^{\prime}-1} M_{s}\left(l+l^{\prime}-1-2 i, i, \eta q^{2 i-l+1} \frac{\left(l+l^{\prime}-1-2 i\right)_{q}}{\left(l^{\prime}\right)_{q}}\right)\right) \\
& \oplus\left(\oplus_{i=t+1}^{l^{\prime}} M_{s}\left(l+l^{\prime}-1-2 i, i, \eta q^{2 i-l+1} \frac{\left(l+l^{\prime}-1-2 i\right)_{q}}{\left(l^{\prime}\right)_{q}}\right)\right) \\
& \oplus\left(\oplus_{i=c\left(l+l^{\prime}-1\right)}^{l-2} s P\left(n+l+l^{\prime}-1-2 i, i\right)\right) \\
& \oplus\left(\oplus_{i=c\left(l+l^{\prime}-1\right)}^{l-1} s P\left(n+l+l^{\prime}-1-2 i, i\right)\right) .
\end{aligned}
$$

Then by an argument similar to the proof of Theorem 3.16, one gets that

$$
\begin{aligned}
V(l, 0) \otimes M_{s}\left(l^{\prime}, 0, \eta\right) \cong & \left(\oplus_{i=c(t)}^{t} s P\left(l+l^{\prime}-1-2 i, i\right)\right) \\
& \oplus\left(\oplus_{t+1 \leqslant i \leqslant l^{\prime}-1} M_{s}\left(l+l^{\prime}-1-2 i, i, \eta q^{2 i-l+1} \frac{\left(l+l^{\prime}-1-2 i\right)_{q}}{\left(l^{\prime}\right)_{q}}\right)\right) \\
& \oplus\left(\oplus_{i=c\left(l+l^{\prime}-1\right)}^{l-1} s P\left(n+l+l^{\prime}-1-2 i, i\right)\right) .
\end{aligned}
$$

This completes the proof.

\section{Tensor product of a projective module with a non-simple module}

In this section, we investigate the tensor product $P \otimes M$ of a non-simple projective indecomposable module $P$ with a non-simple indecomposable module $M$. Since $P \otimes N$ is projective for any module $N, P \otimes M$ is isomorphic to the direct sum of all the tensor products $P \otimes V$, where $V$ ranges all composition factors of $M$. However, the decompositions of all such tensor products $P \otimes V$ are known by Theorems 3.3 and 3.5. which gives rise to the decomposition of $P \otimes M$.

Proposition 4.1. Let $1 \leqslant l \leqslant l^{\prime}<n$ and $r, r^{\prime} \in \mathbb{Z}_{n}$. Assume that $l+l^{\prime} \leqslant n$. Then for all $m \geqslant 1$, we have

$$
\begin{aligned}
& P(l, r) \otimes \Omega^{ \pm m} V\left(l^{\prime}, r^{\prime}\right) \\
\cong & \left(\oplus_{i=0}^{l-1}\left(m+\frac{1+(-1)^{m}}{2}\right) P\left(l+l^{\prime}-1-2 i, r+r^{\prime}+i\right)\right) \\
& \oplus\left(\oplus_{i=l^{\prime}}^{l+l^{\prime}-1}\left(m+\frac{1-(-1)^{m}}{2}\right) P\left(n+l+l^{\prime}-1-2 i, r+r^{\prime}+i\right)\right) \\
& \oplus\left(\oplus_{c\left(l+l^{\prime}-1\right) \leqslant i \leqslant l^{\prime}-1} 2\left(m+\frac{1+(-1)^{m}}{2}\right) P\left(n+l+l^{\prime}-1-2 i, r+r^{\prime}+i\right)\right) \\
& \oplus\left(\oplus_{1 \leqslant i \leqslant c\left(n-l-l^{\prime}\right)} 2\left(m+\frac{1-(-1)^{m}}{2}\right) P\left(l+l^{\prime}-1+2 i, r+r^{\prime}-i\right)\right) .
\end{aligned}
$$

Proof. By Corollary 3.4 and Proposition 3.6 it is enough to show the proposition for $r=r^{\prime}=0$. We may assume that $m$ is odd since the proof is similar when $m$ is even. In this case, there are two exact sequences

$$
\begin{gathered}
0 \rightarrow m V\left(l^{\prime}, 0\right) \rightarrow \Omega^{m} V\left(l^{\prime}, 0\right) \rightarrow(m+1) V\left(n-l^{\prime}, l^{\prime}\right) \rightarrow 0, \\
0 \rightarrow(m+1) V\left(n-l^{\prime}, l^{\prime}\right) \rightarrow \Omega^{-m} V\left(l^{\prime}, 0\right) \rightarrow m V\left(l^{\prime}, 0\right) \rightarrow 0 .
\end{gathered}
$$

Applying $P(l, 0) \otimes$ to the above sequences, one gets the following exact sequences

$$
\begin{aligned}
0 \rightarrow m P(l, 0) \otimes V\left(l^{\prime}, 0\right) & \rightarrow P(l, 0) \otimes \Omega^{m} V\left(l^{\prime}, 0\right) \\
& \rightarrow(m+1) P(l, 0) \otimes V\left(n-l^{\prime}, l^{\prime}\right) \rightarrow 0, \\
0 \rightarrow(m+1) P(l, 0) \otimes V\left(n-l^{\prime}, l^{\prime}\right) & \rightarrow P(l, 0) \otimes \Omega^{-m} V\left(l^{\prime}, 0\right) \\
& \rightarrow m P(l, 0) \otimes V\left(l^{\prime}, 0\right) \rightarrow 0 .
\end{aligned}
$$


TENSOR PRODUCTS OF MODULES OVER DRINFELD DOUBLES OF TAFT ALGEBRAS 21

They are split since $P(l, 0) \otimes V\left(l^{\prime}, 0\right)$ and $P(l, 0) \otimes V\left(n-l^{\prime}, l^{\prime}\right)$ are both projective. By Theorem 3.3, we have

$$
\begin{aligned}
P(l, 0) \otimes V\left(l^{\prime}, 0\right) \cong & \left(\oplus_{i=0}^{l-1} P\left(l+l^{\prime}-1-2 i, i\right)\right) \\
& \oplus\left(\oplus_{c\left(l+l^{\prime}-1\right) \leqslant i \leqslant l^{\prime}-1} 2 P\left(n+l+l^{\prime}-1-2 i, i\right)\right) .
\end{aligned}
$$

By $1 \leqslant l \leqslant l^{\prime}<n$ and $l+l^{\prime} \leqslant n$, one knows that $1 \leqslant l \leqslant n-l^{\prime}<n$ and $l+\left(n-l^{\prime}\right) \leqslant n$. Hence similarly, we have

$$
\begin{aligned}
P(l, 0) \otimes V\left(n-l^{\prime}, l^{\prime}\right) \cong & \left(\oplus_{i=0}^{l-1} P\left(l+n-l^{\prime}-1-2 i, l^{\prime}+i\right)\right) \\
& \oplus\left(\oplus_{c\left(l+n-l^{\prime}-1\right) \leqslant i \leqslant n-l^{\prime}-1} 2 P\left(2 n+l-l^{\prime}-1-2 i, l^{\prime}+i\right)\right) \\
\cong & \left(\oplus_{i=l^{\prime}}^{l+l^{\prime}-1} P\left(n+l+l^{\prime}-1-2 i, i\right)\right) \\
& \oplus\left(\oplus_{1 \leqslant i \leqslant c\left(n-l-l^{\prime}\right)} 2 P\left(l+l^{\prime}-1+2 i,-i\right)\right) .
\end{aligned}
$$

It follows that

$$
\begin{aligned}
& P(l, 0) \otimes \Omega^{m} V\left(l^{\prime}, 0\right) \cong P(l, 0) \otimes \Omega^{-m} V\left(l^{\prime}, 0\right) \\
\cong & \left(\oplus_{i=0}^{l-1} m P\left(l+l^{\prime}-1-2 i, i\right)\right) \\
& \oplus\left(\oplus_{c\left(l+l^{\prime}-1\right) \leqslant i \leqslant l^{\prime}-1} 2 m P\left(n+l+l^{\prime}-1-2 i, i\right)\right) \\
& \oplus\left(\oplus_{i=l^{\prime}}^{l+l^{\prime}-1}(m+1) P\left(n+l+l^{\prime}-1-2 i, i\right)\right) \\
& \oplus\left(\oplus_{1 \leqslant i \leqslant c\left(n-l-l^{\prime}\right)} 2(m+1) P\left(l+l^{\prime}-1+2 i,-i\right)\right) .
\end{aligned}
$$

Corollary 4.2. Let $2 \leqslant l \leqslant l^{\prime}<n$ and $r, r^{\prime} \in \mathbb{Z}_{n}$. Assume that $t=l+l^{\prime}-(n+1) \geqslant$ 0 . Then for all $m \geqslant 1$,

$$
\begin{aligned}
& P(l, r) \otimes \Omega^{ \pm m} V\left(l^{\prime}, r^{\prime}\right) \\
\cong & \left(\oplus_{i=c(t)}^{t} 2\left(m+\frac{1+(-1)^{m}}{2}\right) P\left(l+l^{\prime}-1-2 i, r+r^{\prime}+i\right)\right) \\
& \oplus\left(\oplus_{i=t+1}^{l-1}\left(m+\frac{1+(-1)^{m}}{2}\right) P\left(l+l^{\prime}-1-2 i, r+r^{\prime}+i\right)\right) \\
& \oplus\left(\oplus_{i=l^{\prime}}^{n-1}\left(m+\frac{1-(-1)^{m}}{2}\right) P\left(n+l+l^{\prime}-1-2 i, r+r^{\prime}+i\right)\right) \\
& \oplus\left(\oplus_{c\left(l+l^{\prime}-1\right) \leqslant i \leqslant l^{\prime}-1} 2\left(m+\frac{1+(-1)^{m}}{2}\right) P\left(n+l+l^{\prime}-1-2 i, r+r^{\prime}+i\right)\right) .
\end{aligned}
$$

Proof. By Theorem 3.5, we have

$$
\begin{aligned}
P(l, 0) \otimes V\left(l^{\prime}, 0\right) \cong & \left(\oplus_{i=c(t)}^{t} 2 P\left(l+l^{\prime}-1-2 i, i\right)\right) \\
& \oplus\left(\oplus_{i=t+1}^{l-1} P\left(l+l^{\prime}-1-2 i, i\right)\right) \\
& \oplus\left(\oplus_{c\left(l+l^{\prime}-1\right) \leqslant i \leqslant l^{\prime}-1} 2 P\left(n+l+l^{\prime}-1-2 i, i\right)\right) .
\end{aligned}
$$

By $2 \leqslant l \leqslant l^{\prime}<n$ and $l+l^{\prime} \geqslant n+1$, we have $1 \leqslant n-l^{\prime}<l<n$ and $l+\left(n-l^{\prime}\right) \leqslant n$. Hence by Theorem 3.3, we have

$$
\begin{aligned}
P(l, 0) \otimes V\left(n-l^{\prime}, l^{\prime}\right) & \cong \oplus_{i=0}^{n-l^{\prime}-1} P\left(n+l-l^{\prime}-1-2 i, l^{\prime}+i\right) \\
& \cong \oplus_{i=l^{\prime}}^{n-1} P\left(n+l+l^{\prime}-1-2 i, i\right) .
\end{aligned}
$$

Then the corollary follows from the proof of Proposition 4.1 
Corollary 4.3. Let $1 \leqslant l^{\prime}<l<n$ and $r, r^{\prime} \in \mathbb{Z}_{n}$. Assume that $l+l^{\prime} \leqslant n$. Then for all $m \geqslant 1$,

$$
\begin{aligned}
& P(l, r) \otimes \Omega^{ \pm m} V\left(l^{\prime}, r^{\prime}\right) \\
\cong & \left(\oplus_{i=0}^{l^{\prime}-1}\left(m+\frac{1+(-1)^{m}}{2}\right) P\left(l+l^{\prime}-1-2 i, r+r^{\prime}+i\right)\right) \\
& \oplus\left(\oplus_{i=l}^{l+l^{\prime}-1}\left(m+\frac{1-(-1)^{m}}{2}\right) P\left(n+l+l^{\prime}-1-2 i, r+r^{\prime}+i\right)\right) \\
& \oplus\left(\oplus_{i=c\left(l+l^{\prime}-1\right)}^{l-1} 2\left(m+\frac{1-(-1)^{m}}{2}\right) P\left(n+l+l^{\prime}-1-2 i, r+r^{\prime}+i\right)\right) \\
& \left.\oplus\left(\oplus_{1 \leqslant i \leqslant c\left(n-l-l^{\prime}\right.}\right) 2\left(m+\frac{1-(-1)^{m}}{2}\right) P\left(l+l^{\prime}-1+2 i, r+r^{\prime}-i\right)\right) .
\end{aligned}
$$

Proof. It is similar to Corollary 4.2

Corollary 4.4. Let $2 \leqslant l^{\prime}<l<n$ and $r, r^{\prime} \in \mathbb{Z}_{n}$. Assume that $t=l+l^{\prime}-(n+1) \geqslant$ 0 . Then for all $m \geqslant 1$,

$$
\begin{aligned}
& P(l, r) \otimes \Omega^{ \pm m} V\left(l^{\prime}, r^{\prime}\right) \\
\cong & \left(\oplus_{i=c(t)}^{t} 2\left(m+\frac{1+(-1)^{m}}{2}\right) P\left(l+l^{\prime}-1-2 i, r+r^{\prime}+i\right)\right) \\
& \oplus\left(\oplus_{i=t+1}^{l^{\prime}-1}\left(m+\frac{1+(-1)^{m}}{2}\right) P\left(l+l^{\prime}-1-2 i, r+r^{\prime}+i\right)\right) \\
& \oplus\left(\oplus_{i=l}^{n-1}\left(m+\frac{1-(-1)^{m}}{2}\right) P\left(n+l+l^{\prime}-1-2 i, r+r^{\prime}+i\right)\right) \\
& \oplus\left(\oplus_{i=c\left(l+l^{\prime}-1\right)}^{l-1} 2\left(m+\frac{1-(-1)^{m}}{2}\right) P\left(n+l+l^{\prime}-1-2 i, r+r^{\prime}+i\right)\right) .
\end{aligned}
$$

Proof. It is similar to Corollary 4.2 by using Theorem 3.5 .

Proposition 4.5. Let $1 \leqslant l \leqslant l^{\prime}<n$ and $r, r^{\prime} \in \mathbb{Z}_{n}$. Assume $l+l^{\prime} \leqslant n$. Then

$$
\begin{aligned}
P(l, r) \otimes P\left(l^{\prime}, r^{\prime}\right) \cong & \left(\oplus_{i=0}^{l-1} 2 P\left(l+l^{\prime}-1-2 i, r+r^{\prime}+i\right)\right) \\
& \oplus\left(\oplus_{i=l^{\prime}}^{l^{\prime}+l-1} 2 P\left(n+l+l^{\prime}-1-2 i, r+r^{\prime}+i\right)\right) \\
& \oplus\left(\oplus_{c\left(l^{\prime}+l-1\right) \leqslant i \leqslant l^{\prime}-1} 4 P\left(n+l+l^{\prime}-1-2 i, r+r^{\prime}+i\right)\right) \\
& \oplus\left(\oplus_{1 \leqslant i \leqslant c\left(n-l-l^{\prime}\right)} 4 P\left(l+l^{\prime}-1+2 i, r+r^{\prime}-i\right)\right) .
\end{aligned}
$$

Proof. It is enough to show the proposition for $r=r^{\prime}=0$. By the discussion in Section 2, there is an exact sequence $0 \rightarrow \Omega V\left(l^{\prime}, 0\right) \rightarrow P\left(l^{\prime}, 0\right) \rightarrow V\left(l^{\prime}, 0\right) \rightarrow 0$. Applying $P(l, 0) \otimes$ to the above sequences, one gets another sequence

$$
0 \rightarrow P(l, 0) \otimes \Omega V\left(l^{\prime}, 0\right) \rightarrow P(l, 0) \otimes P\left(l^{\prime}, 0\right) \rightarrow P(l, 0) \otimes V\left(l^{\prime}, 0\right) \rightarrow 0,
$$

which is split since $P(l, 0) \otimes V\left(l^{\prime}, 0\right)$ is projective. Then the proposition follows from Proposition 4.1 and its proof.

Corollary 4.6. Let $2 \leqslant l \leqslant l^{\prime}<n$ and $r, r^{\prime} \in \mathbb{Z}_{n}$. Assume $t=l+l^{\prime}-(n+1) \geqslant 0$. Then

$$
\begin{aligned}
P(l, r) \otimes P\left(l^{\prime}, r^{\prime}\right) \cong & \left(\oplus_{i=c(t)}^{t} 4 P\left(l+l^{\prime}-1-2 i, r+r^{\prime}+i\right)\right) \\
& \oplus\left(\oplus_{i=t+1}^{l-1} 2 P\left(l+l^{\prime}-1-2 i, r+r^{\prime}+i\right)\right) \\
& \oplus\left(\oplus_{i=l^{\prime}}^{n-1} 2 P\left(n+l+l^{\prime}-1-2 i, r+r^{\prime}+i\right)\right) \\
& \oplus\left(\oplus_{c\left(l^{\prime}+l-1\right) \leqslant i \leqslant l^{\prime}-1} 4 P\left(n+l+l^{\prime}-1-2 i, r+r^{\prime}+i\right)\right) .
\end{aligned}
$$

Proof. It is similar to Proposition 4.5, by using Corollary 4.2 and its proof. 
Proposition 4.7. Let $1 \leqslant l, l^{\prime}<n, r, r^{\prime} \in \mathbb{Z}_{n}, \eta \in \mathbb{P}^{1}(k)$ and $s \geqslant 1$. Assume that $l+l^{\prime} \leqslant n$. Let $l_{1}=\min \left\{l, l^{\prime}\right\}$ and $l_{2}=\max \left\{l, l^{\prime}\right\}$. Then

$$
\begin{aligned}
P(l, r) \otimes M_{s}\left(l^{\prime}, r^{\prime}, \eta\right) \cong & \left(\oplus_{i=0}^{l_{1}-1} s P\left(l+l^{\prime}-1-2 i, r+r^{\prime}+i\right)\right) \\
& \oplus\left(\oplus_{i=l_{2}}^{l+l^{\prime}-1} s P\left(n+l+l^{\prime}-1-2 i, r+r^{\prime}+i\right)\right) \\
& \oplus\left(\oplus_{c\left(l+l^{\prime}-1\right) \leqslant i \leqslant l_{2}-1} 2 s P\left(n+l+l^{\prime}-1-2 i, r+r^{\prime}+i\right)\right) \\
& \oplus\left(\oplus_{1 \leqslant i \leqslant c\left(n-l-l^{\prime}\right)} 2 s P\left(l+l^{\prime}-1+2 i, r+r^{\prime}-i\right)\right) .
\end{aligned}
$$

Proof. It is enough to show the proposition for $r=r^{\prime}=0$. By the structure of $M_{s}\left(l^{\prime}, 0, \eta\right)$, we have the following exact sequence

$$
0 \rightarrow s P(l, 0) \otimes V\left(l^{\prime}, 0\right) \rightarrow P(l, 0) \otimes M_{s}\left(l^{\prime}, 0, \eta\right) \rightarrow s P(l, 0) \otimes V\left(n-l^{\prime}, l^{\prime}\right) \rightarrow 0,
$$

which is split as pointed out before. Then the proposition follows from an argument similar to the proof of Proposition 4.1

Corollary 4.8. Let $2 \leqslant l, l^{\prime}<n, r, r^{\prime} \in \mathbb{Z}_{n}, \eta \in \mathbb{P}^{1}(k)$ and $s \geqslant 1$. Assume that $t=l+l^{\prime}-(n+1) \geqslant 0$. Let $l_{1}=\min \left\{l, l^{\prime}\right\}$ and $l_{2}=\max \left\{l, l^{\prime}\right\}$. Then

$$
\begin{aligned}
P(l, r) \otimes M_{s}\left(l^{\prime}, r^{\prime}, \eta\right) \cong & \left(\oplus_{i=c(t)}^{t} 2 s P\left(l+l^{\prime}-1-2 i, r+r^{\prime}+i\right)\right) \\
& \oplus\left(\oplus_{i=t+1}^{l_{1}-1} s P\left(l+l^{\prime}-1-2 i, r+r^{\prime}+i\right)\right) \\
& \oplus\left(\oplus_{i=l_{2}}^{n-1} s P\left(n+l+l^{\prime}-1-2 i, r+r^{\prime}+i\right)\right) \\
& \oplus\left(\oplus_{c\left(l+l^{\prime}-1\right) \leqslant i \leqslant l_{2}-1} 2 s P\left(n+l+l^{\prime}-1-2 i, r+r^{\prime}+i\right)\right) .
\end{aligned}
$$

Proof. It is similar to Proposition 4.7

\section{Tensor product of two modules with Loewy length 2}

In this section, we determine the tensor product of two non-simple non-projective indecomposable modules. We will first consider the tensor product of two string modules.

\subsection{Tensor product of two string modules.}

In this subsection, we determine the tensor product $\Omega^{m} V(l, r) \otimes \Omega^{s} V\left(l^{\prime}, r\right)$ of two string modules, where $m, s \in \mathbb{Z}$. By [13, p.438], $\Omega^{m} V(l, r) \otimes \Omega^{s} V\left(l^{\prime}, r\right) \cong$ $\Omega^{m+s}\left(V(l, r) \otimes V\left(l^{\prime}, 0\right)\right) \oplus P$ for some projective module $P$. The first term on the right side of the isomorphism is easily determined by Proposition 3.1. However, the projective summand $P$ is not easy to determine in general. For $m \geqslant 0$, we determine the tensor product by the induction on $m$ through the exact sequence

$$
\begin{aligned}
0 \rightarrow \Omega^{m+1} V(l, r) \otimes \Omega^{s} V\left(l^{\prime}, r^{\prime}\right) & \rightarrow P\left(\Omega^{m} V(l, r)\right) \otimes \Omega^{s} V\left(l^{\prime}, r^{\prime}\right) \\
& \rightarrow \Omega^{m} V(l, r) \otimes \Omega^{s} V\left(l^{\prime}, r^{\prime}\right) \rightarrow 0 .
\end{aligned}
$$

Here we use the following Lemma 5.1, which is obvious, and the fact that $\Omega(M \oplus$ $P) \cong \Omega M$ for any module $M$ and projective module $P$. Then applying the duality $(-)^{*}$, one achieves the corresponding decompositions for $m<0$.

Lemma 5.1. Let $0 \rightarrow N \rightarrow P \rightarrow M \rightarrow 0$ be an exact sequence of modules over a finite dimensional algebra $A$, where $P$ is projective. Then $P \cong P(M) \oplus Q$ for some submodule $Q$ of $P$. Moreover, $Q$ is unique up to isomorphism, and $N \cong \Omega M \oplus Q$. 
Proposition 5.2. Let $1 \leqslant l \leqslant l^{\prime}<n, r, r^{\prime} \in \mathbb{Z}_{n}, m \geqslant 0$ and $s \geqslant 1$. Assume that $l+l^{\prime} \leqslant n$, and let $m_{1}=\min \{m, s\}$ and $m_{2}=\max \{m, s\}$. Let $P$ be the module

$$
\begin{aligned}
& \left(\oplus_{c\left(l^{\prime}+l-1\right) \leqslant i \leqslant l^{\prime}-1}\left(m+\frac{1-(-1)^{m}}{2}\right)\left(s+\frac{1+(-1)^{s}}{2}\right) P\left(n+l+l^{\prime}-1-2 i, r+r^{\prime}+i\right)\right) \\
& \oplus\left(\oplus_{1 \leqslant i \leqslant c\left(n-l-l^{\prime}\right)}\left(m+\frac{1-(-1)^{m}}{2}\right)\left(s+\frac{1-(-1)^{s}}{2}\right) P\left(l+l^{\prime}-1+2 i, r+r^{\prime}-i\right)\right) .
\end{aligned}
$$

(1) If $m+s$ is even, then

$$
\begin{aligned}
\Omega^{m} V(l, r) \otimes \Omega^{s} V\left(l^{\prime}, r^{\prime}\right) \cong & \left(\oplus_{i=0}^{l-1} \Omega^{m+s} V\left(l+l^{\prime}-1-2 i, r+r^{\prime}+i\right)\right) \\
& \oplus\left(\oplus_{i=0}^{l-1} m s P\left(l+l^{\prime}-1-2 i, r+r^{\prime}+i\right)\right) \oplus P
\end{aligned}
$$

and

$$
\begin{aligned}
& \Omega^{m} V(l, r) \otimes \Omega^{-s} V\left(l^{\prime}, r^{\prime}\right) \\
\cong & \left(\oplus_{i=0}^{l-1} \Omega^{m-s} V\left(l+l^{\prime}-1-2 i, r+r^{\prime}+i\right)\right) \\
& \oplus\left(\oplus_{i=l^{\prime}}^{l+l^{\prime}-1} m_{1}\left(m_{2}+1\right) P\left(n+l+l^{\prime}-1-2 i, r+r^{\prime}+i\right)\right) \oplus P .
\end{aligned}
$$

(2) If $m+s$ is odd, then

$$
\begin{aligned}
\Omega^{m} V(l, r) \otimes \Omega^{s} V\left(l^{\prime}, r^{\prime}\right) \cong & \left(\oplus_{i=0}^{l-1} \Omega^{m+s} V\left(l+l^{\prime}-1-2 i, r+r^{\prime}+i\right)\right) \\
& \oplus\left(\oplus_{i=l^{\prime}}^{l+l^{\prime}-1} m s P\left(n+l+l^{\prime}-1-2 i, r+r^{\prime}+i\right)\right) \oplus P
\end{aligned}
$$

and

$$
\begin{aligned}
\Omega^{m} V(l, r) \otimes \Omega^{-s} V\left(l^{\prime}, r^{\prime}\right) \cong & \left(\oplus_{i=0}^{l-1} \Omega^{m-s} V\left(l+l^{\prime}-1-2 i, r+r^{\prime}+i\right)\right) \\
& \oplus\left(\oplus_{i=0}^{l-1} m_{1}\left(m_{2}+1\right) P\left(l+l^{\prime}-1-2 i, r+r^{\prime}+i\right)\right) \oplus P .
\end{aligned}
$$

Proof. It is enough to show the proposition for $r=r^{\prime}=0$. We prove it by the induction on $m$. For $m=0$, it follows from Proposition 3.6. Now let $m>0$. We only consider the case that $m$ and $s$ are both even since the proofs are similar for the other cases. In this case, we have an exact sequence

$$
\begin{aligned}
0 \rightarrow \Omega^{m} V(l, 0) \otimes \Omega^{ \pm s} V\left(l^{\prime}, 0\right) & \rightarrow m P(n-l, l) \otimes \Omega^{ \pm s} V\left(l^{\prime}, 0\right) \\
& \rightarrow \Omega^{m-1} V(l, 0) \otimes \Omega^{ \pm s} V\left(l^{\prime}, 0\right) \rightarrow 0 .
\end{aligned}
$$

From $1 \leqslant l \leqslant l^{\prime}<n$ and $l+l^{\prime} \leqslant n$, one gets that $1 \leqslant l^{\prime} \leqslant n-l<n$ and $n-l+l^{\prime} \geqslant n$. Moreover, $n-l+l^{\prime}-(n+1)=l^{\prime}-l-1$. Hence by Corollary 4.4 together with Proposition 4.1 for $l+l^{\prime}=n$ and $l=l^{\prime}$, Corollary 4.2 for $l+l^{\prime}=n$ and $l<l^{\prime}$, and Corollary 4.3 for $l+l^{\prime}<n$ and $l=l^{\prime}$, we have

$$
\begin{aligned}
& m P(n-l, l) \otimes \Omega^{ \pm s} V\left(l^{\prime}, 0\right) \\
\cong & \left(\oplus_{c\left(l^{\prime}-l-1\right) \leqslant i \leqslant l^{\prime}-l-1} 2 m(s+1) P\left(n-l+l^{\prime}-1-2 i, l+i\right)\right) \\
& \oplus\left(\oplus_{i=l^{\prime}-l}^{l^{\prime}-1} m(s+1) P\left(n-l+l^{\prime}-1-2 i, l+i\right)\right) \\
& \oplus\left(\oplus_{i=n-l}^{n-1} m s P\left(2 n-l+l^{\prime}-1-2 i, l+i\right)\right) \\
& \oplus\left(\oplus_{c\left(n-l+l^{\prime}-1\right) \leqslant i \leqslant n-l-1} 2 m s P\left(2 n-l+l^{\prime}-1-2 i, l+i\right)\right) \\
\cong & \left(\oplus_{c\left(l+l^{\prime}-1\right) \leqslant i \leqslant l^{\prime}-1} 2 m(s+1) P\left(n+l+l^{\prime}-1-2 i, i\right)\right) \\
& \oplus\left(\oplus_{i=l^{\prime}}^{l+l^{\prime}-1} m(s+1) P\left(n+l+l^{\prime}-1-2 i, i\right)\right) \\
& \oplus\left(\oplus_{i=0}^{l-1} m s P\left(l+l^{\prime}-1-2 i, i\right)\right) \\
& \oplus\left(\oplus_{1 \leqslant i \leqslant c\left(n-l-l^{\prime}\right)} 2 m s P\left(l+l^{\prime}-1+2 i,-i\right)\right) .
\end{aligned}
$$


TENSOR PRODUCTS OF MODULES OVER DRINFELD DOUBLES OF TAFT ALGEBRAS 25

Note that $m-1+s$ and $m-1$ are both odd. By the induction hypothesis, we have

$$
\begin{aligned}
& \Omega^{m-1} V(l, 0) \otimes \Omega^{s} V\left(l^{\prime}, 0\right) \\
\cong & \left(\oplus_{i=0}^{l-1} \Omega^{m-1+s} V\left(l+l^{\prime}-1-2 i, i\right)\right) \\
& \oplus\left(\oplus_{i=l^{\prime}}^{l+l^{\prime}-1}(m-1) s P\left(n+l+l^{\prime}-1-2 i, i\right)\right) \\
& \oplus\left(\oplus_{c\left(l^{\prime}+l-1\right) \leqslant i \leqslant l^{\prime}-1} m(s+1) P\left(n+l+l^{\prime}-1-2 i, i\right)\right) \\
& \oplus\left(\oplus_{1 \leqslant i \leqslant c\left(n-l-l^{\prime}\right)} m s P\left(l+l^{\prime}-1+2 i,-i\right)\right) .
\end{aligned}
$$

It is easy to check that $\oplus_{i=l^{\prime}}^{l+l^{\prime}-1}(m+s) P\left(n+l+l^{\prime}-1-2 i, i\right)$ is a projective cover of $\oplus_{i=0}^{l-1} \Omega^{m-1+s} V\left(l+l^{\prime}-1-2 i, i\right)$. Hence we have

$$
\begin{aligned}
& m P(n-l, l) \otimes \Omega^{s} V\left(l^{\prime}, 0\right) \\
\cong & P\left(\Omega^{m-1} V(l, 0) \otimes \Omega^{s} V\left(l^{\prime}, 0\right)\right) \\
& \oplus\left(\oplus_{c\left(l+l^{\prime}-1\right) \leqslant i \leqslant l^{\prime}-1} m(s+1) P\left(n+l+l^{\prime}-1-2 i, i\right)\right) \\
& \oplus\left(\oplus_{i=0}^{l-1} m s P\left(l+l^{\prime}-1-2 i, i\right)\right) \\
& \oplus\left(\oplus_{1 \leqslant i \leqslant c\left(n-l-l^{\prime}\right)} m s P\left(l+l^{\prime}-1+2 i,-i\right)\right) .
\end{aligned}
$$

It follows from Lemma 5.1 that

$$
\begin{aligned}
\Omega^{m} V(l, 0) \otimes \Omega^{s} V\left(l^{\prime}, 0\right) \cong & \left(\oplus_{i=0}^{l-1} \Omega^{m+s} V\left(l+l^{\prime}-1-2 i, i\right)\right) \\
& \oplus\left(\oplus_{i=0}^{l-1} m s P\left(l+l^{\prime}-1-2 i, i\right)\right) \\
& \oplus\left(\oplus_{c\left(l+l^{\prime}-1\right) \leqslant i \leqslant l^{\prime}-1} m(s+1) P\left(n+l+l^{\prime}-1-2 i, i\right)\right) \\
& \oplus\left(\oplus_{1 \leqslant i \leqslant c\left(n-l-l^{\prime}\right)} m s P\left(l+l^{\prime}-1+2 i,-i\right)\right) .
\end{aligned}
$$

If $s \geqslant m$, then $s>m-1$. Hence by the induction hypothesis, we have

$$
\begin{aligned}
& \Omega^{m-1} V(l, 0) \otimes \Omega^{-s} V\left(l^{\prime}, 0\right) \\
\cong & \left(\oplus_{i=0}^{l-1} \Omega^{m-1-s} V\left(l+l^{\prime}-1-2 i, i\right)\right) \\
& \oplus\left(\oplus_{i=0}^{l-1}(m-1)(s+1) P\left(l+l^{\prime}-1-2 i, i\right)\right) \\
& \oplus\left(\oplus_{c\left(l+l^{\prime}-1\right) \leqslant i \leqslant l^{\prime}-1} m(s+1) P\left(n+l+l^{\prime}-1-2 i, i\right)\right) \\
& \oplus\left(\oplus_{1 \leqslant i \leqslant c\left(n-l-l^{\prime}\right)} m s P\left(l+l^{\prime}-1+2 i,-i\right)\right) .
\end{aligned}
$$

In this case, $s-m+1 \geqslant 1$ is odd. Hence $\oplus_{i=0}^{l-1}(s-m+1) P\left(l+l^{\prime}-1-2 i, i\right)$ is a projective cover of $\oplus_{i=0}^{l-1} \Omega^{m-1-s} V\left(l+l^{\prime}-1-2 i, i\right)$. Thus, a similar argument as above shows that

$$
\begin{aligned}
\Omega^{m} V(l, 0) \otimes \Omega^{-s} V\left(l^{\prime}, 0\right) \cong & \left(\oplus_{i=0}^{l-1} \Omega^{m-s} V\left(l+l^{\prime}-1-2 i, i\right)\right) \\
& \oplus\left(\oplus_{i=l^{\prime}}^{l+l^{\prime}-1} m(s+1) P\left(n+l+l^{\prime}-1-2 i, i\right)\right) \\
& \oplus\left(\oplus_{c\left(l+l^{\prime}-1\right) \leqslant i \leqslant l^{\prime}-1} m(s+1) P\left(n+l+l^{\prime}-1-2 i, i\right)\right) \\
& \oplus\left(\oplus_{1 \leqslant i \leqslant c\left(n-l-l^{\prime}\right)} m s P\left(l+l^{\prime}-1+2 i,-i\right)\right) .
\end{aligned}
$$

If $m>s$, then $m-1 \geqslant s$. Hence by the induction hypothesis, we have

$$
\begin{aligned}
& \Omega^{m-1} V(l, 0) \otimes \Omega^{-s} V\left(l^{\prime}, 0\right) \\
\cong & \left(\oplus_{i=0}^{l-1} \Omega^{m-1-s} V\left(l+l^{\prime}-1-2 i, i\right)\right) \\
& \oplus\left(\oplus_{i=0}^{l-1} s m P\left(l+l^{\prime}-1-2 i, i\right)\right) \\
& \oplus\left(\oplus_{c\left(l+l^{\prime}-1\right) \leqslant i \leqslant l^{\prime}-1} m(s+1) P\left(n+l+l^{\prime}-1-2 i, i\right)\right) \\
& \oplus\left(\oplus_{1 \leqslant i \leqslant c\left(n-l-l^{\prime}\right)} m s P\left(l+l^{\prime}-1+2 i,-i\right)\right) .
\end{aligned}
$$


In this case, $m-1-s \geqslant 0$ is odd. Hence $\oplus_{i=l^{\prime}}^{l+l^{\prime}-1}(m-s) P\left(n+l+l^{\prime}-1-2 i, i\right)$ is a projective cover of $\oplus_{i=0}^{l-1} \Omega^{m-1-s} V\left(l+l^{\prime}-1-2 i, i\right)$ as above, and so similarly,

$$
\begin{aligned}
\Omega^{m} V(l, 0) \otimes \Omega^{-s} V\left(l^{\prime}, 0\right) \cong & \left(\oplus_{i=0}^{l-1} \Omega^{m-s} V\left(l+l^{\prime}-1-2 i, i\right)\right) \\
& \oplus\left(\oplus_{i=l^{\prime}}^{l+l^{\prime}-1} s(m+1) P\left(n+l+l^{\prime}-1-2 i, i\right)\right) \\
& \oplus\left(\oplus_{c\left(l+l^{\prime}-1\right) \leqslant i \leqslant l^{\prime}-1} m(s+1) P\left(n+l+l^{\prime}-1-2 i, i\right)\right) \\
& \oplus\left(\oplus_{1 \leqslant i \leqslant c\left(n-l-l^{\prime}\right)} m s P\left(l+l^{\prime}-1+2 i,-i\right)\right),
\end{aligned}
$$

as desired. This completes the proof.

Corollary 5.3. Let $1 \leqslant l \leqslant l^{\prime}<n, r, r^{\prime} \in \mathbb{Z}_{n}$ and $s, m \geqslant 1$. Assume that $l+l^{\prime} \leqslant n$, and let $m_{1}=\min \{m, s\}$ and $m_{2}=\max \{m, s\}$. Let $P$ be the module

$$
\begin{aligned}
& \left(\oplus_{c\left(l^{\prime}+l-1\right) \leqslant i \leqslant l^{\prime}-1}\left(m+\frac{1-(-1)^{m}}{2}\right)\left(s+\frac{1+(-1)^{s}}{2}\right) P\left(n+l+l^{\prime}-1-2 i, r+r^{\prime}+i\right)\right) \\
& \oplus\left(\oplus_{1 \leqslant i \leqslant c\left(n-l-l^{\prime}\right)}\left(m+\frac{1-(-1)^{m}}{2}\right)\left(s+\frac{1-(-1)^{s}}{2}\right) P\left(l+l^{\prime}-1+2 i, r+r^{\prime}-i\right)\right) .
\end{aligned}
$$

(1) If $m+s$ is even, then

$$
\begin{aligned}
\Omega^{-m} V(l, r) \otimes \Omega^{-s} V\left(l^{\prime}, r^{\prime}\right) \cong & \left(\oplus_{i=0}^{l-1} \Omega^{-(m+s)} V\left(l+l^{\prime}-1-2 i, r+r^{\prime}+i\right)\right) \\
& \oplus\left(\oplus_{i=0}^{l-1} m s P\left(l+l^{\prime}-1-2 i, r+r^{\prime}+i\right)\right) \oplus P
\end{aligned}
$$

and

$$
\begin{aligned}
& \Omega^{-m} V(l, r) \otimes \Omega^{s} V\left(l^{\prime}, r^{\prime}\right) \\
\cong & \left(\oplus_{i=0}^{l-1} \Omega^{s-m} V\left(l+l^{\prime}-1-2 i, r+r^{\prime}+i\right)\right) \\
& \oplus\left(\oplus_{i=l^{\prime}}^{l+l^{\prime}-1} m_{1}\left(m_{2}+1\right) P\left(n+l+l^{\prime}-1-2 i, r+r^{\prime}+i\right)\right) \oplus P .
\end{aligned}
$$

(2) If $m+s$ is odd, then

$$
\begin{aligned}
\Omega^{-m} V(l, r) \otimes \Omega^{-s} V\left(l^{\prime}, r^{\prime}\right) \cong & \left(\oplus_{i=0}^{l-1} \Omega^{-(m+s)} V\left(l+l^{\prime}-1-2 i, r+r^{\prime}+i\right)\right) \\
& \oplus\left(\oplus_{i=l^{\prime}}^{l+l^{\prime}-1} m s P\left(n+l+l^{\prime}-1-2 i, r+r^{\prime}+i\right)\right) \oplus P
\end{aligned}
$$

and

$$
\begin{aligned}
\Omega^{-m} V(l, r) \otimes \Omega^{s} V\left(l^{\prime}, r^{\prime}\right) \cong & \left(\oplus_{i=0}^{l-1} \Omega^{s-m} V\left(l+l^{\prime}-1-2 i, r+r^{\prime}+i\right)\right) \\
& \oplus\left(\oplus_{i=0}^{l-1} m_{1}\left(m_{2}+1\right) P\left(l+l^{\prime}-1-2 i, r+r^{\prime}+i\right)\right) \oplus P .
\end{aligned}
$$

Proof. Applying the duality $(-)^{*}$ to the isomorphisms in Proposition 5.2 , the corollary follows from Lemma 3.12

Proposition 5.4. Let $1 \leqslant l \leqslant l^{\prime}<n, r, r^{\prime} \in \mathbb{Z}_{n}, m \geqslant 0$ and $s \geqslant 1$. Assume that $t=l+l^{\prime}-(n+1) \geqslant 0$, and let $m_{1}=\min \{m, s\}$ and $m_{2}=\max \{m, s\}$. Let

$$
\begin{gathered}
P=\left(\oplus_{i=c(t)}^{t}\left(m+\frac{1+(-1)^{m}}{2}\right)\left(s+\frac{1+(-1)^{s}}{2}\right) P\left(l+l^{\prime}-1-2 i, r+r^{\prime}+i\right)\right) \\
\oplus\left(\oplus_{c\left(l+l^{\prime}-1\right) \leqslant i \leqslant l^{\prime}-1}\left(m+\frac{1-(-1)^{m}}{2}\right)\left(s+\frac{1+(-1)^{s}}{2}\right) P\left(n+l+l^{\prime}-1-2 i, r+r^{\prime}+i\right)\right) .
\end{gathered}
$$

(1) If $m+s$ is even, then

$$
\begin{aligned}
\Omega^{m} V(l, r) \otimes \Omega^{s} V\left(l^{\prime}, r^{\prime}\right) \cong & \left(\oplus_{i=t+1}^{l-1} \Omega^{m+s} V\left(l+l^{\prime}-1-2 i, r+r^{\prime}+i\right)\right) \\
& \oplus\left(\oplus_{i=t+1}^{l-1} m s P\left(l+l^{\prime}-1-2 i, r+r^{\prime}+i\right)\right) \oplus P
\end{aligned}
$$

and

$$
\begin{aligned}
& \Omega^{m} V(l, r) \otimes \Omega^{-s} V\left(l^{\prime}, r^{\prime}\right) \\
\cong & \left(\oplus_{i=t+1}^{l-1} \Omega^{m-s} V\left(l+l^{\prime}-1-2 i, r+r^{\prime}+i\right)\right) \\
& \oplus\left(\oplus_{i=l^{\prime}}^{n-1} m_{1}\left(m_{2}+1\right) P\left(n+l+l^{\prime}-1-2 i, r+r^{\prime}+i\right)\right) \oplus P .
\end{aligned}
$$


(2) If $m+s$ is odd, then

$$
\begin{aligned}
\Omega^{m} V(l, r) \otimes \Omega^{s} V\left(l^{\prime}, r^{\prime}\right) \cong & \left(\oplus_{i=t+1}^{l-1} \Omega^{m+s} V\left(l+l^{\prime}-1-2 i, r+r^{\prime}+i\right)\right) \\
& \oplus\left(\oplus_{i=l^{\prime}}^{n-1} m s P\left(n+l+l^{\prime}-1-2 i, r+r^{\prime}+i\right)\right) \oplus P
\end{aligned}
$$

and

$$
\begin{aligned}
& \Omega^{m} V(l, r) \otimes \Omega^{-s} V\left(l^{\prime}, r^{\prime}\right) \\
\cong & \left(\oplus_{i=t+1}^{l-1} \Omega^{m-s} V\left(l+l^{\prime}-1-2 i, r+r^{\prime}+i\right)\right) \\
& \oplus\left(\oplus_{i=t+1}^{l-1} m_{1}\left(m_{2}+1\right) P\left(l+l^{\prime}-1-2 i, r+r^{\prime}+i\right)\right) \oplus P .
\end{aligned}
$$

Proof. It is similar to Proposition 5.2, where we use Proposition 3.7 for $m=0$.

Corollary 5.5. Let $1 \leqslant l \leqslant l^{\prime}<n, r, r^{\prime} \in \mathbb{Z}_{n}$ and $s, m \geqslant 1$. Assume that $t=l+l^{\prime}-(n+1) \geqslant 0$, and let $m_{1}=\min \{m, s\}$ and $m_{2}=\max \{m, s\}$. Let

$$
\begin{gathered}
P=\left(\oplus_{i=c(t)}^{t}\left(m+\frac{1+(-1)^{m}}{2}\right)\left(s+\frac{1+(-1)^{s}}{2}\right) P\left(l+l^{\prime}-1-2 i, r+r^{\prime}+i\right)\right) \\
\oplus\left(\oplus_{c\left(l+l^{\prime}-1\right) \leqslant i \leqslant l^{\prime}-1}\left(m+\frac{1-(-1)^{m}}{2}\right)\left(s+\frac{1+(-1)^{s}}{2}\right) P\left(n+l+l^{\prime}-1-2 i, r+r^{\prime}+i\right)\right) .
\end{gathered}
$$

(1) If $m+s$ is even, then

$$
\begin{aligned}
\Omega^{-m} V(l, r) \otimes \Omega^{-s} V\left(l^{\prime}, r^{\prime}\right) \cong & \left(\oplus_{i=t+1}^{l-1} \Omega^{-(m+s)} V\left(l+l^{\prime}-1-2 i, r+r^{\prime}+i\right)\right) \\
& \oplus\left(\oplus_{i=t+1}^{l-1} m s P\left(l+l^{\prime}-1-2 i, r+r^{\prime}+i\right)\right) \oplus P
\end{aligned}
$$

and

$$
\begin{aligned}
& \Omega^{-m} V(l, r) \otimes \Omega^{s} V\left(l^{\prime}, r^{\prime}\right) \\
\cong & \left(\oplus_{i=t+1}^{l-1} \Omega^{s-m} V\left(l+l^{\prime}-1-2 i, r+r^{\prime}+i\right)\right) \\
& \oplus\left(\oplus_{i=l^{\prime}}^{n-1} m_{1}\left(m_{2}+1\right) P\left(n+l+l^{\prime}-1-2 i, r+r^{\prime}+i\right)\right) \oplus P .
\end{aligned}
$$

(3) If $m+s$ is odd, then

$$
\begin{aligned}
\Omega^{-m} V(l, r) \otimes \Omega^{-s} V\left(l^{\prime}, r^{\prime}\right) \cong & \left(\oplus_{i=t+1}^{l-1} \Omega^{-(s+m)} V\left(l+l^{\prime}-1-2 i, r+r^{\prime}+i\right)\right) \\
& \oplus\left(\oplus_{i=l^{\prime}}^{n-1} m s P\left(n+l+l^{\prime}-1-2 i, r+r^{\prime}+i\right)\right) \oplus P
\end{aligned}
$$

and

$$
\begin{aligned}
& \Omega^{-m} V(l, r) \otimes \Omega^{s} V\left(l^{\prime}, r^{\prime}\right) \\
\cong & \left(\oplus_{i=t+1}^{l-1} \Omega^{s-m} V\left(l+l^{\prime}-1-2 i, r+r^{\prime}+i\right)\right) \\
& \oplus\left(\oplus_{i=t+1}^{l-1} m_{1}\left(m_{2}+1\right) P\left(l+l^{\prime}-1-2 i, r+r^{\prime}+i\right)\right) \oplus P .
\end{aligned}
$$

Proof. Applying the duality $(-)^{*}$ to the isomorphisms in Proposition 5.4, the corollary follows from Lemmas 3.12 .

\subsection{Tensor product of a string module with a band module.}

In this subsection, we determine the tensor product $M=\Omega^{m} V(l, r) \otimes M_{s}\left(l^{\prime}, r^{\prime}, \eta\right)$ of a string module with a band module. Using the same method as in the last subsection (replacing $\Omega^{s} V\left(l^{\prime}, r^{\prime}\right)$ by $M_{s}\left(l^{\prime}, r^{\prime}, \eta\right)$ there), we can determine $M$.

Proposition 5.6. Let $1 \leqslant l \leqslant l^{\prime}<n, r, r^{\prime} \in \mathbb{Z}_{n}, \eta \in \mathbb{P}^{1}(k), s \geqslant 1$ and $m \geqslant 0$. Assume that $l+l^{\prime} \leqslant n$. 
(1) If $m$ is odd, then

$$
\begin{aligned}
& \Omega^{m} V(l, r) \otimes M_{s}\left(l^{\prime}, r^{\prime}, \eta\right) \\
\cong & \left(\oplus_{i=l^{\prime}}^{l+l^{\prime}-1} M_{s}\left(n+l+l^{\prime}-1-2 i, r+r^{\prime}+i,-\eta q^{l^{\prime}} \frac{\left(2 i-l-l^{\prime}+1\right)_{q}}{\left(l^{\prime}\right)_{q}}\right)\right) \\
& \oplus\left(\oplus_{i=0}^{l-1} m s P\left(l+l^{\prime}-1-2 i, r+r^{\prime}+i\right)\right) \\
& \oplus\left(\oplus_{c\left(l+l^{\prime}-1\right) \leqslant i \leqslant l^{\prime}-1}(m+1) s P\left(n+l+l^{\prime}-1-2 i, r+r^{\prime}+i\right)\right) \\
& \oplus\left(\oplus_{1 \leqslant i \leqslant c\left(n-l-l^{\prime}\right)}(m+1) s P\left(l+l^{\prime}-1+2 i, r+r^{\prime}-i\right)\right) .
\end{aligned}
$$

(2) If $m$ is even, then

$$
\begin{aligned}
& \Omega^{m} V(l, r) \otimes M_{s}\left(l^{\prime}, r^{\prime}, \eta\right) \\
\cong & \left(\oplus_{i=0}^{l-1} M_{s}\left(l+l^{\prime}-1-2 i, r+r^{\prime}+i, \eta q^{2 i-l+1} \frac{\left(l+l^{\prime}-1-2 i\right)_{q}}{\left(l^{\prime}\right)_{q}}\right)\right) \\
& \oplus\left(\oplus_{i=l^{\prime}}^{l+l^{\prime}-1} m s P\left(n+l+l^{\prime}-1-2 i, r+r^{\prime}+i\right)\right) \\
& \oplus\left(\oplus_{c\left(l+l^{\prime}-1\right) \leqslant i \leqslant l^{\prime}-1} m s P\left(n+l+l^{\prime}-1-2 i, r+r^{\prime}+i\right)\right) \\
& \oplus\left(\oplus_{1 \leqslant i \leqslant c\left(n-l-l^{\prime}\right)} m s P\left(l+l^{\prime}-1+2 i, r+r^{\prime}-i\right)\right) .
\end{aligned}
$$

Proof. It is enough to show the proposition for $r=r^{\prime}=0$. We prove it by the induction on $m$. For $m=0$, it follows from Theorem 3.16. Now let $m>0$.

We only consider the case that $m$ is odd since the proof is similar when $m$ is even. In this case, $m-1$ is even, and hence there is an exact sequence

$$
\begin{aligned}
0 \rightarrow \Omega^{m} V(l, 0) \otimes M_{s}\left(l^{\prime}, 0, \eta\right) & \rightarrow m P(l, 0) \otimes M_{s}\left(l^{\prime}, 0, \eta\right) \\
& \rightarrow \Omega^{m-1} V(l, 0) \otimes M_{s}\left(l^{\prime}, 0, \eta\right) \rightarrow 0 .
\end{aligned}
$$

By the induction hypothesis, we have

$$
\begin{aligned}
& \Omega^{m-1} V(l, 0) \otimes M_{s}\left(l^{\prime}, 0, \eta\right) \\
\cong & \left(\oplus_{i=0}^{l-1} M_{s}\left(l+l^{\prime}-1-2 i, i, \eta q^{2 i-l+1} \frac{\left(l+l^{\prime}-1-2 i\right)_{q}}{\left(l^{\prime}\right)_{q}}\right)\right) \\
& \oplus\left(\oplus_{i=l^{\prime}}^{+l l^{\prime}-1}(m-1) s P\left(n+l+l^{\prime}-1-2 i, i\right)\right) \\
& \oplus\left(\oplus_{c\left(l+l^{\prime}-1\right) \leqslant i \leqslant l^{\prime}-1}(m-1) s P\left(n+l+l^{\prime}-1-2 i, i\right)\right) \\
& \oplus\left(\oplus_{1 \leqslant i \leqslant c\left(n-l-l^{\prime}\right)}(m-1) s P\left(l+l^{\prime}-1+2 i,-i\right)\right) .
\end{aligned}
$$

Note that $\oplus_{i=l^{\prime}}^{l+l^{\prime}} s P\left(n+l+l^{\prime}-1-2 i, i\right)$ is a projective cover of $\oplus_{i=0}^{l-1} M_{s}\left(l+l^{\prime}-\right.$ $\left.1-2 i, i, \eta q^{2 i-l+1} \frac{\left(l+l^{\prime}-1-2 i\right)_{q}}{\left(l^{\prime}\right)_{q}}\right)$ and

$$
\begin{aligned}
& \Omega\left(\oplus_{i=0}^{l-1} M_{s}\left(l+l^{\prime}-1-2 i, i, \eta q^{2 i-l+1} \frac{\left(l+l^{\prime}-1-2 i\right)_{q}}{\left(l^{\prime}\right)_{q}}\right)\right) \\
\cong & \oplus_{i=l^{\prime}}^{l+l^{\prime}-1} M_{s}\left(n+l+l^{\prime}-1-2 i, i,-\eta q^{l^{\prime}} \frac{\left(2 i-l-l^{\prime}+1\right)_{q}}{\left(l^{\prime}\right)_{q}}\right) .
\end{aligned}
$$

Hence by Proposition 4.7, we have

$$
\begin{aligned}
m P(l, 0) \otimes M_{s}\left(l^{\prime}, 0, \eta\right) \cong & \left(\oplus_{i=0}^{l-1} m s P\left(l+l^{\prime}-1-2 i, i\right)\right) \\
& \oplus\left(\oplus_{i=l^{\prime}}^{l+l^{\prime}-1} m s P\left(n+l+l^{\prime}-1-2 i, i\right)\right) \\
& \oplus\left(\oplus_{c\left(l+l^{\prime}-1\right) \leqslant i \leqslant l^{\prime}-1} 2 m s P\left(n+l+l^{\prime}-1-2 i, i\right)\right) \\
& \oplus\left(\oplus_{1 \leqslant i \leqslant c\left(n-l-l^{\prime}\right)} 2 m s P\left(l+l^{\prime}-1+2 i,-i\right)\right) \\
\cong & P\left(\Omega^{m-1} V(l, 0) \otimes M_{s}\left(l^{\prime}, 0, \eta\right)\right) \\
& \oplus\left(\oplus_{i=0}^{l-1} m s P\left(l+l^{\prime}-1-2 i, i\right)\right) \\
& \oplus\left(\oplus_{c\left(l+l^{\prime}-1\right) \leqslant i \leqslant l^{\prime}-1}(m+1) s P\left(n+l+l^{\prime}-1-2 i, i\right)\right) \\
& \oplus\left(\oplus_{1 \leqslant i \leqslant c\left(n-l-l^{\prime}\right)}(m+1) s P\left(l+l^{\prime}-1+2 i,-i\right)\right) .
\end{aligned}
$$


Then it follows from Lemma 5.1 that

$$
\begin{aligned}
\Omega^{m} V(l, 0) \otimes M_{s}\left(l^{\prime}, 0, \eta\right) \cong & \left(\oplus_{i=l^{\prime}}^{l+l^{\prime}-1} M_{s}\left(n+l+l^{\prime}-1-2 i, i,-\eta q^{l^{\prime}} \frac{\left(2 i-l-l^{\prime}+1\right)_{q}}{\left(l^{\prime}\right)_{q}}\right)\right) \\
& \oplus\left(\oplus_{i=0}^{l-1} m s P\left(l+l^{\prime}-1-2 i, i\right)\right) \\
& \oplus\left(\oplus_{c\left(l+l^{\prime}-1\right) \leqslant i \leqslant l^{\prime}-1}(m+1) s P\left(n+l+l^{\prime}-1-2 i, i\right)\right) \\
& \oplus\left(\oplus_{1 \leqslant i \leqslant c\left(n-l-l^{\prime}\right)}(m+1) s P\left(l+l^{\prime}-1+2 i,-i\right)\right),
\end{aligned}
$$

as desired. This completes the proof.

Corollary 5.7. Let $1 \leqslant l \leqslant l^{\prime}<n, r, r^{\prime} \in \mathbb{Z}_{n}, \eta \in \mathbb{P}^{1}(k)$ and $s, m \geqslant 1$. Assume that $l+l^{\prime} \leqslant n$.

(1) If $m$ is odd, then

$$
\begin{aligned}
& \Omega^{-m} V(l, r) \otimes M_{s}\left(l^{\prime}, r^{\prime}, \eta\right) \\
\cong & \left(\oplus_{i=l^{\prime}}^{l+l^{\prime}-1} M_{s}\left(n+l+l^{\prime}-1-2 i, r+r^{\prime}+i,-\eta q^{l^{\prime}} \frac{\left(2 i-l-l^{\prime}+1\right)_{q}}{\left(l^{\prime}\right)_{q}}\right)\right) \\
& \oplus\left(\oplus_{i=l^{\prime}}^{++l^{\prime}-1} m s P\left(n+l+l^{\prime}-1-2 i, r+r^{\prime}+i\right)\right) \\
& \oplus\left(\oplus_{c\left(l+l^{\prime}-1\right) \leqslant i \leqslant l^{\prime}-1}(m+1) s P\left(n+l+l^{\prime}-1-2 i, r+r^{\prime}+i\right)\right) \\
& \oplus\left(\oplus_{1 \leqslant i \leqslant c\left(n-l-l^{\prime}\right)}(m+1) s P\left(l+l^{\prime}-1+2 i, r+r^{\prime}-i\right)\right) .
\end{aligned}
$$

(2) If $m$ is even, then

$$
\begin{aligned}
& \Omega^{-m} V(l, r) \otimes M_{s}\left(l^{\prime}, r^{\prime}, \eta\right) \\
\cong & \left(\oplus_{i=0}^{l-1} M_{s}\left(l+l^{\prime}-1-2 i, r+r^{\prime}+i, \eta q^{2 i-l+1} \frac{\left(l+l^{\prime}-1-2 i\right)_{q}}{\left(l^{\prime}\right)_{q}}\right)\right) \\
& \oplus\left(\oplus_{i=0}^{l-1} m s P\left(l+l^{\prime}-1-2 i, r+r^{\prime}+i\right)\right) \\
& \oplus\left(\oplus_{c\left(l+l^{\prime}-1\right) \leqslant i \leqslant l^{\prime}-1} m s P\left(n+l+l^{\prime}-1-2 i, r+r^{\prime}+i\right)\right) \\
& \oplus\left(\oplus_{1 \leqslant i \leqslant c\left(n-l-l^{\prime}\right)} \operatorname{ms} P\left(l+l^{\prime}-1+2 i, r+r^{\prime}-i\right)\right) .
\end{aligned}
$$

Proof. It is enough to show the corollary for $r=r^{\prime}=0$. Since $1 \leqslant l \leqslant l^{\prime}<n$ and $l+l^{\prime} \leqslant n$, we have $1 \leqslant l \leqslant n-l^{\prime}<n$ and $l+n-l^{\prime} \leqslant n$.

(1) Assume that $m$ is odd. Then by Proposition 5.6, we have

$$
\begin{aligned}
& \Omega^{m} V(l, 1-l) \otimes M_{s}\left(n-l^{\prime}, 1,-\eta q^{l^{\prime}}\right) \\
\cong & \left(\oplus_{i=n-l^{\prime}}^{l+n-1} M_{s}\left(2 n+l-l^{\prime}-1-2 i, 2-l+i, \eta \frac{\left(2 i-l-n+l^{\prime}+1\right)_{q}}{\left(n-l^{\prime}\right)_{q}}\right)\right) \\
& \oplus\left(\oplus_{i=0}^{l-1} m s P\left(l+n-l^{\prime}-1-2 i, 2-l+i\right)\right) \\
& \oplus\left(\oplus_{c\left(l+n-l^{\prime}-1\right) \leqslant i \leqslant n-l^{\prime}-1}(m+1) s P\left(2 n+l-l^{\prime}-1-2 i, 2-l+i\right)\right) \\
& \oplus\left(\oplus_{1 \leqslant i \leqslant c\left(l^{\prime}-l\right)}(m+1) s P\left(l+n-l^{\prime}-1+2 i, 2-l-i\right)\right) .
\end{aligned}
$$

Then applying the duality $(-)^{*}$ to the above isomorphism and using Lemmas 3.12 and 3.13, a tedious but standard computation shows that

$$
\begin{aligned}
& \Omega^{-m} V(l, 0) \otimes M_{s}\left(l^{\prime}, 0, \eta\right) \\
\cong & \left(\oplus_{i=l^{\prime}}^{l+l^{\prime}-1} M_{s}\left(n+l+l^{\prime}-1-2 i, i,-\eta q^{l^{\prime}} \frac{\left(2 i-l-l^{\prime}+1\right)_{q}}{\left(l^{\prime}\right)_{q}}\right)\right) \\
& \oplus\left(\oplus_{i=l^{\prime}}^{l+l^{\prime}-1} m s P\left(n+l+l^{\prime}-1-2 i, i\right)\right) \\
& \oplus\left(\oplus_{c\left(l+l^{\prime}-1\right) \leqslant i \leqslant l^{\prime}-1}(m+1) s P\left(n+l+l^{\prime}-1-2 i, i\right)\right) \\
& \oplus\left(\oplus_{1 \leqslant i \leqslant c\left(n-l-l^{\prime}\right)}(m+1) s P\left(l+l^{\prime}-1+2 i,-i\right)\right) .
\end{aligned}
$$

(2) It is similar to Part (1). 
Proposition 5.8. Let $2 \leqslant l \leqslant l^{\prime}<n, r, r^{\prime} \in \mathbb{Z}_{n}, \eta \in \mathbb{P}^{1}(k), s \geqslant 1$ and $m \geqslant 0$. Assume that $t=l+l^{\prime}-(n+1) \geqslant 0$.

(1) If $m$ is odd, then

$$
\begin{aligned}
& \Omega^{m} V(l, r) \otimes M_{s}\left(l^{\prime}, r^{\prime}, \eta\right) \\
\cong & \left(\oplus_{i=l^{\prime}}^{n-1} M_{s}\left(n+l+l^{\prime}-1-2 i, r+r^{\prime}+i,-\eta q^{l^{\prime}} \frac{\left(2 i-l-l^{\prime}+1\right)_{q}}{\left(l^{\prime}\right)_{q}}\right)\right) \\
& \oplus\left(\oplus_{i=t+1}^{l-1} m s P\left(l+l^{\prime}-1-2 i, r+r^{\prime}+i\right)\right) \\
& \oplus\left(\oplus_{i=c(t)}^{t} m s P\left(l+l^{\prime}-1-2 i, r+r^{\prime}+i\right)\right) \\
& \oplus\left(\oplus_{c\left(l+l^{\prime}-1\right) \leqslant i \leqslant l^{\prime}-1}(m+1) s P\left(n+l+l^{\prime}-1-2 i, r+r^{\prime}+i\right)\right)
\end{aligned}
$$

(2) If $m$ is even, then

$$
\begin{aligned}
& \Omega^{m} V(l, r) \otimes M_{s}\left(l^{\prime}, r^{\prime}, \eta\right) \\
\cong & \left(\oplus_{i=t+1}^{l-1} M_{s}\left(l+l^{\prime}-1-2 i, r+r^{\prime}+i, \eta q^{2 i-l+1} \frac{\left(l+l^{\prime}-1-2 i\right)_{q}}{\left(l^{\prime}\right)_{q}}\right)\right) \\
& \oplus\left(\oplus_{i=l^{\prime}}^{n-1} m s P\left(n+l+l^{\prime}-1-2 i, r+r^{\prime}+i\right)\right) \\
& \oplus\left(\oplus_{i=c(t)}^{t}(m+1) s P\left(l+l^{\prime}-1-2 i, r+r^{\prime}+i\right)\right) \\
& \oplus\left(\oplus_{c\left(l+l^{\prime}-1\right) \leqslant i \leqslant l^{\prime}-1} m s P\left(n+l+l^{\prime}-1-2 i, r+r^{\prime}+i\right)\right) .
\end{aligned}
$$

Proof. It is similar to Proposition[5.6] where we use Theorem 3.17 for $m=0$.

Corollary 5.9. Let $1 \leqslant l^{\prime}<l<n, r, r^{\prime} \in \mathbb{Z}_{n}, \eta \in \mathbb{P}^{1}(k)$ and $s, m \geqslant 1$. Assume that $l+l^{\prime} \leqslant n$.

(1) If $m$ is odd, then

$$
\begin{aligned}
& \Omega^{-m} V(l, r) \otimes M_{s}\left(l^{\prime}, r^{\prime}, \eta\right) \\
\cong & \left(\oplus_{i=l}^{l+l^{\prime}-1} M_{s}\left(n+l+l^{\prime}-1-2 i, r+r^{\prime}+i,-\eta q^{l^{\prime}} \frac{\left(2 i-l-l^{\prime}+1\right)_{q}}{\left(l^{\prime}\right)_{q}}\right)\right) \\
& \oplus\left(\oplus_{i=l}^{l+l^{\prime}-1} m s P\left(n+l+l^{\prime}-1-2 i, r+r^{\prime}+i\right)\right) \\
& \oplus\left(\oplus_{i=c\left(l+l^{\prime}-1\right)}^{l-1} m s P\left(n+l+l^{\prime}-1-2 i, r+r^{\prime}+i\right)\right) \\
& \oplus\left(\oplus_{1 \leqslant i \leqslant c\left(n-l-l^{\prime}\right)}(m+1) s P\left(l+l^{\prime}-1+2 i, r+r^{\prime}-i\right)\right) .
\end{aligned}
$$

(2) If $m$ is even, then

$$
\begin{aligned}
& \Omega^{-m} V(l, r) \otimes M_{s}\left(l^{\prime}, r^{\prime}, \eta\right) \\
\cong & \left(\oplus_{i=0}^{l^{\prime}-1} M_{s}\left(l+l^{\prime}-1-2 i, r+r^{\prime}+i, \eta q^{2 i-l+1} \frac{\left(l+l^{\prime}-1-2 i\right)_{q}}{\left(l^{\prime}\right)_{q}}\right)\right) \\
& \oplus\left(\oplus_{i=0}^{l^{\prime}-1} m s P\left(l+l^{\prime}-1-2 i, r+r^{\prime}+i\right)\right) \\
& \oplus\left(\oplus_{i=c\left(l+l^{\prime}-1\right)}^{l-1}(m+1) s P\left(n+l+l^{\prime}-1-2 i, r+r^{\prime}+i\right)\right) \\
& \oplus\left(\oplus_{1 \leqslant i \leqslant c\left(n-l-l^{\prime}\right)} m s P\left(l+l^{\prime}-1+2 i, r+r^{\prime}-i\right)\right) .
\end{aligned}
$$

Proof. It is similar to Corollary 5.7 by using the duality $(-)^{*}$, Lemmas 3.123 .13 and Proposition 5.8

Proposition 5.10. Let $1 \leqslant l^{\prime}<l<n, r, r^{\prime} \in \mathbb{Z}_{n}, \eta \in \mathbb{P}^{1}(k), s \geqslant 1$ and $m \geqslant 0$. Assume that $l+l^{\prime} \leqslant n$. 
TENSOR PRODUCTS OF MODULES OVER DRINFELD DOUBLES OF TAFT ALGEBRAS 31

(1) If $m$ is odd, then

$$
\begin{aligned}
& \Omega^{m} V(l, r) \otimes M_{s}\left(l^{\prime}, r^{\prime}, \eta\right) \\
\cong & \left(\oplus_{i=l}^{l+l^{\prime}-1} M_{s}\left(n+l+l^{\prime}-1-2 i, r+r^{\prime}+i,-\eta q^{l^{\prime}} \frac{\left(2 i-l-l^{\prime}+1\right)_{q}}{\left(l^{\prime}\right)_{q}}\right)\right) \\
& \oplus\left(\oplus_{i=0}^{l^{\prime}-1} m s P\left(l+l^{\prime}-1-2 i, r+r^{\prime}+i\right)\right) \\
& \oplus\left(\oplus_{i=c\left(l+l^{\prime}-1\right)}^{l-1} m s P\left(n+l+l^{\prime}-1-2 i, r+r^{\prime}+i\right)\right) \\
& \oplus\left(\oplus_{1 \leqslant i \leqslant c\left(n-l-l^{\prime}\right)}(m+1) s P\left(l+l^{\prime}-1+2 i, r+r^{\prime}-i\right)\right) .
\end{aligned}
$$

(2) If $m$ is even, then

$$
\begin{aligned}
& \Omega^{m} V(l, r) \otimes M_{s}\left(l^{\prime}, r^{\prime}, \eta\right) \\
\cong & \left(\oplus_{i=0}^{l^{\prime}-1} M_{s}\left(l+l^{\prime}-1-2 i, r+r^{\prime}+i, \eta q^{2 i-l+1} \frac{\left(l+l^{\prime}-1-2 i\right)_{q}}{\left(l^{\prime}\right)_{q}}\right)\right) \\
& \oplus\left(\oplus_{i=l}^{l+l^{\prime}-1} m s P\left(n+l+l^{\prime}-1-2 i, r+r^{\prime}+i\right)\right) \\
& \oplus\left(\oplus_{i=c\left(l+l^{\prime}-1\right)}^{l-1}(m+1) s P\left(n+l+l^{\prime}-1-2 i, r+r^{\prime}+i\right)\right) \\
& \oplus\left(\oplus_{1 \leqslant i \leqslant c\left(n-l-l^{\prime}\right)} m s P\left(l+l^{\prime}-1+2 i, r+r^{\prime}-i\right)\right) .
\end{aligned}
$$

Proof. It is similar to Proposition 5.6, where we use Theorem 3.16 for $m=0$.

Corollary 5.11. Let $2 \leqslant l \leqslant l^{\prime}<n, r, r^{\prime} \in \mathbb{Z}_{n}, \eta \in \mathbb{P}^{1}(k)$ and $s, m \geqslant 1$. Assume that $t=l+l^{\prime}-(n+1) \geqslant 0$.

(1) If $m$ is odd, then

$$
\begin{aligned}
& \Omega^{-m} V(l, r) \otimes M_{s}\left(l^{\prime}, r^{\prime}, \eta\right) \\
\cong & \left(\oplus_{i=l^{\prime}}^{n-1} M_{s}\left(n+l+l^{\prime}-1-2 i, r+r^{\prime}+i,-\eta q^{l^{\prime}} \frac{\left(2 i-l-l^{\prime}+1\right)_{q}}{\left(l^{\prime}\right)_{q}}\right)\right) \\
& \oplus\left(\oplus_{i=l^{\prime}}^{n-1} m s P\left(n+l+l^{\prime}-1-2 i, r+r^{\prime}+i\right)\right) \\
& \oplus\left(\oplus_{i=c(t)}^{t} m s P\left(l+l^{\prime}-1-2 i, r+r^{\prime}+i\right)\right) \\
& \oplus\left(\oplus_{c\left(l+l^{\prime}-1\right) \leqslant i \leqslant l^{\prime}-1}(m+1) s P\left(n+l+l^{\prime}-1-2 i, r+r^{\prime}+i\right)\right) .
\end{aligned}
$$

(2) If $m$ is even, then

$$
\begin{aligned}
& \Omega^{-m} V(l, r) \otimes M_{s}\left(l^{\prime}, r^{\prime}, \eta\right) \\
\cong & \left(\oplus_{i=t+1}^{l-1} M_{s}\left(l+l^{\prime}-1-2 i, r+r^{\prime}+i, \eta q^{2 i-l+1} \frac{\left(l+l^{\prime}-1-2 i\right)_{q}}{\left(l^{\prime}\right)_{q}}\right)\right) \\
& \oplus\left(\oplus_{i=t+1}^{l-1} m s P\left(l+l^{\prime}-1-2 i, r+r^{\prime}+i\right)\right) \\
& \oplus\left(\oplus_{i=c(t)}^{t}(m+1) s P\left(l+l^{\prime}-1-2 i, r+r^{\prime}+i\right)\right) \\
& \oplus\left(\oplus_{c\left(l+l^{\prime}-1\right) \leqslant i \leqslant l^{\prime}-1} m s P\left(n+l+l^{\prime}-1-2 i, r+r^{\prime}+i\right)\right) .
\end{aligned}
$$

Proof. It is similar to Corollary 5.7 by using the duality $(-)^{*}$, Lemmas 3.123 .13 and Proposition 5.10 .

Proposition 5.12. Let $2 \leqslant l^{\prime}<l<n, r, r^{\prime} \in \mathbb{Z}_{n}, \eta \in \mathbb{P}^{1}(k), s \geqslant 1$ and $m \geqslant 0$. Assume that $t=l+l^{\prime}-(n+1) \geqslant 0$.

(1) If $m$ is odd, then

$$
\begin{aligned}
& \Omega^{m} V(l, r) \otimes M_{s}\left(l^{\prime}, r^{\prime}, \eta\right) \\
\cong & \left(\oplus_{i=l}^{n-1} M_{s}\left(n+l+l^{\prime}-1-2 i, r+r^{\prime}+i,-\eta q^{l^{\prime}} \frac{\left(2 i-l-l^{\prime}+1\right)_{q}}{\left(l^{\prime}\right)_{q}}\right)\right) \\
& \oplus\left(\oplus_{i=t+1}^{l^{\prime}-1} m s P\left(l+l^{\prime}-1-2 i, r+r^{\prime}+i\right)\right) \\
& \oplus\left(\oplus_{i=c(t)}^{t} m s P\left(l+l^{\prime}-1-2 i, r+r^{\prime}+i\right)\right) \\
& \oplus\left(\oplus_{i=c\left(l+l^{\prime}-1\right)}^{l-1} m s P\left(n+l+l^{\prime}-1-2 i, r+r^{\prime}+i\right)\right) .
\end{aligned}
$$


(2) If $m$ is even, then

$$
\begin{aligned}
& \Omega^{m} V(l, r) \otimes M_{s}\left(l^{\prime}, r^{\prime}, \eta\right) \\
\cong & \left(\oplus_{i=t+1}^{l^{\prime}-1} M_{s}\left(l+l^{\prime}-1-2 i, r+r^{\prime}+i, \eta q^{2 i-l+1} \frac{\left(l+l^{\prime}-1-2 i\right)_{q}}{\left(l^{\prime}\right)_{q}}\right)\right) \\
& \oplus\left(\oplus_{i=l}^{n-1} m s P\left(n+l+l^{\prime}-1-2 i, r+r^{\prime}+i\right)\right) \\
& \oplus\left(\oplus_{i=c(t)}^{t}(m+1) s P\left(l+l^{\prime}-1-2 i, r+r^{\prime}+i\right)\right) \\
& \oplus\left(\oplus_{i=c\left(l+l^{\prime}-1\right)}^{l-1}(m+1) s P\left(n+l+l^{\prime}-1-2 i, r+r^{\prime}+i\right)\right) .
\end{aligned}
$$

Proof. It is similar to Proposition [5.6, where we use Theorem 3.17 for $m=0$.

Corollary 5.13. Let $2 \leqslant l^{\prime}<l<n, r, r^{\prime} \in \mathbb{Z}_{n}, \eta \in \mathbb{P}^{1}(k)$ and $s, m \geqslant 1$. Assume that $t=l+l^{\prime}-(n+1) \geqslant 0$.

(1) If $m$ is odd, then

$$
\begin{aligned}
& \Omega^{-m} V(l, r) \otimes M_{s}\left(l^{\prime}, r^{\prime}, \eta\right) \\
\cong & \left(\oplus_{i=l}^{n-1} M_{s}\left(n+l+l^{\prime}-1-2 i, r+r^{\prime}+i,-\eta q^{l^{\prime}} \frac{\left(2 i-l-l^{\prime}+1\right)_{q}}{\left(l^{\prime}\right)_{q}}\right)\right) \\
& \oplus\left(\oplus_{i=l}^{n-1} m s P\left(n+l+l^{\prime}-1-2 i, r+r^{\prime}+i\right)\right) \\
& \oplus\left(\oplus_{i=c(t)}^{t} m s P\left(l+l^{\prime}-1-2 i, r+r^{\prime}+i\right)\right) \\
& \oplus\left(\oplus_{i=c\left(l+l^{\prime}-1\right)}^{l-1} m s P\left(n+l+l^{\prime}-1-2 i, r+r^{\prime}+i\right)\right) .
\end{aligned}
$$

(2) If $m$ is even, then

$$
\begin{aligned}
& \Omega^{-m} V(l, r) \otimes M_{s}\left(l^{\prime}, r^{\prime}, \eta\right) \\
\cong & \left(\oplus_{i=t+1}^{l^{\prime}-1} M_{s}\left(l+l^{\prime}-1-2 i, r+r^{\prime}+i, \eta q^{2 i-l+1} \frac{\left(l+l^{\prime}-1-2 i\right)_{q}}{\left(l^{\prime}\right)_{q}}\right)\right) \\
& \oplus\left(\oplus_{i=t+1}^{l^{\prime}-1} m s P\left(l+l^{\prime}-1-2 i, r+r^{\prime}+i\right)\right) \\
& \oplus\left(\oplus_{i=c(t)}^{t}(m+1) s P\left(l+l^{\prime}-1-2 i, r+r^{\prime}+i\right)\right) \\
& \oplus\left(\oplus_{i=c\left(l+l^{\prime}-1\right)}^{l-1}(m+1) s P\left(n+l+l^{\prime}-1-2 i, r+r^{\prime}+i\right)\right) .
\end{aligned}
$$

Proof. It is similar to Corollary 5.7 by using the duality $(-)^{*}$, Lemmas 3.123 .13 and Proposition 5.12 .

\subsection{Tensor product of two band modules.}

In this subsection, we investigate the tensor product $M=M_{m}(l, r, \alpha) \otimes M_{s}\left(l^{\prime}, r^{\prime}, \eta\right)$ of two band modules. By [13, any non-projective indecomposable summand of $M$ must be a band module. The module on an example with $m=s=1$ is displayed in [13] for the special case $n=d=6$. We will determine $M$ for two cases $\alpha q^{1-l^{\prime}}\left(l^{\prime}\right)_{q} \neq \eta q^{1-l}(l)_{q}$ and $\alpha q^{1-l^{\prime}}\left(l^{\prime}\right)_{q}=\eta q^{1-l}(l)_{q}$, respectively.

In the case of $\alpha q^{1-l^{\prime}}\left(l^{\prime}\right)_{q} \neq \eta q^{1-l}(l)_{q}$, we show that $M$ is projective, and determine the decomposition of $M$ by the inductions on $m+s$ and $l+l^{\prime}$. We first determine $M$ for $l=l^{\prime}=1$ by the induction on $m+s$. Here we use the last exact sequence in Section 2 Then tensoring by $V(2,0)$, we determine $M$ by the induction on $l+l^{\prime}$.

In the case of $\alpha q^{1-l^{\prime}}\left(l^{\prime}\right)_{q}=\eta q^{1-l}(l)_{q}$, we first determine $M$ for $l+l^{\prime} \leqslant n$ by the induction on $l+l^{\prime}$. For $l=l^{\prime}=1$, we use the exact sequence

$$
\begin{aligned}
0 \rightarrow M_{m}(1, r, \eta) \otimes M_{s}\left(1, r^{\prime}, \eta\right) & \rightarrow \Omega^{m} V \otimes M_{s}\left(1, r^{\prime}, \eta\right) \\
& \stackrel{f}{\rightarrow} V(n-1, r+1) \otimes M_{s}\left(1, r^{\prime}, \eta\right) \rightarrow 0,
\end{aligned}
$$


where $V=V(1, r)$ for $m$ being odd, and $V=V(n-l, r+l)$ for $m$ being even. The decompositions of the middle and right terms are known. We show that the nonprojective summand of the middle term is contained in the kernel of $f$, which gives rise to the decomposition of the left term by Lemma 5.1. For the induction step, we use tensoring with $V(2,0)$. Finally, applying the duality $(-)^{*}$ to the decomposition of $M$ for $l+l^{\prime}<n$, one gets the decomposition of $M$ for $l+l^{\prime} \geqslant n$.

Now we first consider the case of $\alpha q^{1-l^{\prime}}\left(l^{\prime}\right)_{q} \neq \eta q^{1-l}(l)_{q}$.

Lemma 5.14. Let $\eta \in \mathbb{P}^{1}(k)$ and $M \in \mathcal{M}$. Assume that $M$ fits into an exact sequence

$$
0 \rightarrow M_{1}(1,0, \eta) \rightarrow M \rightarrow M_{1}(n-1,1,-\eta q) \rightarrow 0 .
$$

Then $M \cong M_{1}(1,0, \eta) \oplus M_{1}(n-1,1,-\eta q)$ or $M \cong P(1,0)$.

Proof. From the exact sequence $0 \rightarrow M_{1}(1,0, \eta) \rightarrow P(1,0) \rightarrow M_{1}(n-1,1,-\eta q) \rightarrow$ 0 , one gets a long exact sequence

$$
\begin{aligned}
0 & \rightarrow \operatorname{Hom}_{H_{n}(1, q)}\left(M_{1}(n-1,1,-\eta q), M_{1}(1,0, \eta)\right) \\
& \rightarrow \operatorname{Hom}_{H_{n}(1, q)}\left(P(1,0), M_{1}(1,0, \eta)\right) \rightarrow \operatorname{Hom}_{H_{n}(1, q)}\left(M_{1}(1,0, \eta), M_{1}(1,0, \eta)\right) \\
& \rightarrow \operatorname{Ext}_{H_{n}(1, q)}^{1}\left(M_{1}(n-1,1,-\eta q), M_{1}(1,0, \eta)\right) \rightarrow 0 .
\end{aligned}
$$

A straightforward verification shows that $\operatorname{Hom}_{H_{n}(1, q)}\left(M_{1}(n-1,1,-\eta q), M_{1}(1,0, \eta)\right)$, $\operatorname{Hom}_{H_{n}(1, q)}\left(P(1,0), M_{1}(1,0, \eta)\right)$ and $\operatorname{Hom}_{H_{n}(1, q)}\left(M_{1}(1,0, \eta), M_{1}(1,0, \eta)\right)$ are all one dimensional over $k$. Hence $\operatorname{Ext}_{H_{n}(1, q)}^{1}\left(M_{1}(n-1,1,-\eta q), M_{1}(1,0, \eta)\right) \cong k$. It follows that $M \cong M_{1}(1,0, \eta) \oplus M_{1}(n-1,1,-\eta q)$ or $M \cong P(1,0)$.

Lemma 5.15. Let $r, r^{\prime} \in \mathbb{Z}_{n}, \alpha, \eta \in \mathbb{P}^{1}(k)$ and $s, m \geqslant 1$. Assume $\alpha \neq \eta$. Then

$$
M_{m}(1, r, \alpha) \otimes M_{s}\left(1, r^{\prime}, \eta\right) \cong \oplus_{i=1}^{c(n)} m s P\left(2 i-1, r+r^{\prime}-i+1\right) .
$$

Proof. It is enough to show the lemma for $r=r^{\prime}=0$. We prove it by the induction on $m+s$. We first assume that $m+s=2$. Then $m=s=1$. Let $M=M_{1}(1,0, \alpha) \otimes M_{1}(1,0, \eta)$. Applying $M_{1}(1,0, \alpha) \otimes$ to the exact sequence $0 \rightarrow V(1,0) \rightarrow M_{1}(1,0, \eta) \rightarrow V(n-1,1) \rightarrow 0$, one gets another exact sequence $0 \rightarrow M_{1}(1,0, \alpha) \otimes V(1,0) \rightarrow M \rightarrow M_{1}(1,0, \alpha) \otimes V(n-1,1) \rightarrow 0$. By Theorem 3.16 we have

$$
\begin{aligned}
& M_{1}(1,0, \alpha) \otimes V(n-1,1) \\
\cong & \left.M_{1}(n-1,1,-\alpha q)\right) \oplus\left(\oplus_{i=c(n-1)}^{n-2} P(2 n-1-2 i, i+1)\right) \\
\cong & M_{1}(n-1,1,-\alpha q) \oplus\left(\oplus_{i=2}^{c(n)} P(2 i-1,1-i)\right) .
\end{aligned}
$$

By $M_{1}(1,0, \alpha) \otimes V(1,0) \cong M_{1}(1,0, \alpha)$, it follows that there exist two submodules $M_{1}$ and $M_{2}$ of $M$ with $M=M_{1} \oplus M_{2}$ such that $M_{2} \cong \oplus_{i=2}^{c(n)} P(2 i-1,1-i)$ and $M_{1}$ fits an exact sequence

$$
0 \rightarrow M_{1}(1,0, \alpha) \rightarrow M_{1} \rightarrow M_{1}(n-1,1,-\alpha q) \rightarrow 0 .
$$

Then by Lemma 5.14, $M_{1} \cong M_{1}(1,0, \alpha) \oplus M_{1}(n-1,1,-\alpha q)$ or $M_{1} \cong P(1,0)$. Since $M_{1}(1,0, \alpha) \otimes M_{1}(1,0, \eta) \cong M_{1}(1,0, \eta) \otimes M_{1}(1,0, \alpha)$, a similar argument as above shows that $M=N_{1} \oplus N_{2}$, where $N_{1}$ and $N_{2}$ are submodules of $M, N_{2} \cong$ $\oplus_{i=2}^{c(n)} P(2 i-1,1-i)$, and $N_{1} \cong M_{1}(1,0, \eta) \oplus M_{1}(n-1,1,-\eta q)$ or $N_{1} \cong P(1,0)$. Since $M=M_{1} \oplus M_{2}=N_{1} \oplus N_{2}$ and $M_{2} \cong N_{2}$, it follows from Krull-Schmidt Theorem that 
$M_{1} \cong N_{1}$. However, $M_{1}(1,0, \alpha) \oplus M_{1}(n-1,1,-\alpha q) \varsubsetneqq M_{1}(1,0, \eta) \oplus M_{1}(n-1,1,-\eta q)$ by $\alpha \neq \eta$. Therefore, $M_{1} \cong N_{1} \cong P(1,0)$. Thus, we have $M_{1}(1,0, \alpha) \otimes M_{1}(1,0, \eta) \cong$ $\oplus_{i=1}^{c(n)} P(2 i-1,1-i)$.

Now assume that $m+s>2$. We may assume that $m \geqslant 2$ without losing the generality. Then there is an exact sequence

$$
\begin{aligned}
0 \rightarrow M_{m-1}(1,0, \alpha) \otimes M_{s}(1,0, \eta) & \rightarrow M_{m}(1,0, \alpha) \otimes M_{s}(1,0, \eta) \\
& \rightarrow M_{1}(1,0, \alpha) \otimes M_{s}(1,0, \eta) \rightarrow 0 .
\end{aligned}
$$

By the induction hypothesis, $M_{1}(1,0, \alpha) \otimes M_{s}(1,0, \eta)$ is projective, and so the above exact sequence is split. Again by the induction hypothesis, we have

$$
\begin{aligned}
& M_{m}(1,0, \alpha) \otimes M_{s}(1,0, \eta) \\
\cong & M_{m-1}(1,0, \alpha) \otimes M_{s}(1,0, \eta) \oplus M_{1}(1,0, \alpha) \otimes M_{s}(1,0, \eta) \\
\cong & \left(\oplus_{i=1}^{c(n)}(m-1) s P(2 i-1,1-i)\right) \oplus\left(\oplus_{i=1}^{c(n)} s P(2 i-1,1-i)\right) \\
\cong & \oplus_{i=1}^{c(n)} m s P(2 i-1,1-i) .
\end{aligned}
$$

This completes the proof.

Proposition 5.16. Let $1 \leqslant l \leqslant l^{\prime}<n, r, r^{\prime} \in \mathbb{Z}_{n}, \alpha, \eta \in \mathbb{P}^{1}(k)$ and $s, m \geqslant 1$. Assume that $\alpha \neq \eta$. Then

$$
\begin{aligned}
& M_{m}\left(l, r, \alpha q^{1-l}(l)_{q}\right) \otimes M_{s}\left(l^{\prime}, r^{\prime}, \eta q^{1-l^{\prime}}\left(l^{\prime}\right)_{q}\right) \\
\cong & \left(\oplus_{i=1}^{c\left(n+l-l^{\prime}\right)} m s P\left(l^{\prime}-l-1+2 i, r+r^{\prime}+l-i\right)\right) \\
& \oplus\left(\oplus_{c\left(l+l^{\prime}-1\right) \leqslant i \leqslant l^{\prime}-1} m s P\left(n+l+l^{\prime}-1-2 i, r+r^{\prime}+i\right)\right) .
\end{aligned}
$$

Proof. It is enough to show the proposition for $r=r^{\prime}=0$. We prove it by the induction on $l+l^{\prime}$. For $l+l^{\prime}=2$, it follows from Lemma 5.15. Now let $l+l^{\prime}>2$.

We only consider the case of $l<l^{\prime}$ since the proof is similar for $l=l^{\prime}$. In this case, $l^{\prime}-1 \geqslant l$. By the induction hypothesis, we have

$$
\begin{aligned}
& M_{m}\left(l, 0, \alpha q^{1-l}(l)_{q}\right) \otimes M_{s}\left(l^{\prime}-1,0, \eta q^{2-l^{\prime}}\left(l^{\prime}-1\right)_{q}\right) \otimes V(2,0) \\
\cong & \left(\oplus_{i=1}^{c\left(n+l-l^{\prime}+1\right)} m s P\left(l^{\prime}-l-2+2 i, l-i\right) \otimes V(2,0)\right) \\
& \oplus\left(\oplus_{c\left(l+l^{\prime}-2\right) \leqslant i \leqslant l^{\prime}-2} m s P\left(n+l+l^{\prime}-2-2 i, i\right) \otimes V(2,0)\right) .
\end{aligned}
$$

If $l^{\prime}=2$, then $l=1$. Hence by Lemma 3.11 and Theorem 3.17, we have

$$
\begin{aligned}
& M_{m}(1,0, \alpha) \otimes M_{s}(1,0, \eta) \otimes V(2,0) \\
\cong & M_{m}(1,0, \alpha) \otimes M_{s}\left(2,0, \eta q^{-1}(2)_{q}\right) \oplus s M_{m}(1,0, \alpha) \otimes V(n, 1) \\
\cong & M_{m}(1,0, \alpha) \otimes M_{s}\left(2,0, \eta q^{-1}(2)_{q}\right) \oplus\left(\oplus_{i=1}^{c(n-1)} m s P(2 i, 1-i)\right) \oplus m s V(n, 1) .
\end{aligned}
$$

In this case, using Proposition 3.1. Theorems 3.3 and 3.5 a straightforward computation (for $n$ to be even and odd, respectively) shows that

$$
\oplus_{i=1}^{c(n)} P(2 i-1,1-i) \otimes V(2,0) \cong\left(\oplus_{i=1}^{c(n-1)} 2 P(2 i, 1-i)\right) \oplus 2 V(n, 1) .
$$

Thus, it follows from Krull-Schmidt Theorem that

$$
M_{m}(1,0, \alpha) \otimes M_{s}\left(2,0, \eta q^{-1}(2)_{q}\right) \cong\left(\oplus_{i=1}^{c(n-1)} m s P(2 i, 1-i)\right) \oplus m s V(n, 1)
$$


TENSOR PRODUCTS OF MODULES OVER DRINFELD DOUBLES OF TAFT ALGEBRAS 35

as desired. If $l^{\prime}>2$ and $l \leqslant l^{\prime}-2$, then by Lemma 3.15 (or Theorem 3.16) and the induction hypothesis, we have

$$
\begin{aligned}
& M_{m}\left(l, 0, \alpha q^{1-l}(l)_{q}\right) \otimes M_{s}\left(l^{\prime}-1,0, \eta q^{2-l^{\prime}}\left(l^{\prime}-1\right)_{q}\right) \otimes V(2,0) \\
\cong & M_{m}\left(l, 0, \alpha q^{1-l}(l)_{q}\right) \otimes M_{s}\left(l^{\prime}, 0, \eta q^{1-l^{\prime}}\left(l^{\prime}\right)_{q}\right) \\
& \oplus M_{m}\left(l, 0, \alpha q^{1-l}(l)_{q}\right) \otimes M_{s}\left(l^{\prime}-2,1, \eta q^{-l^{\prime}}\left(l^{\prime}-2\right)_{q}\right) \\
\cong & M_{m}\left(l, 0, \alpha q^{1-l}(l)_{q}\right) \otimes M_{s}\left(l^{\prime}, 0, \eta q^{1-l^{\prime}}\left(l^{\prime}\right)_{q}\right) \\
& \oplus\left(\oplus_{i=1}^{c\left(n+l-l^{\prime}+2\right)} m s P\left(l^{\prime}-l-3+2 i, 1+l-i\right)\right) \\
& \oplus\left(\oplus_{c\left(l+l^{\prime}-3\right) \leqslant i \leqslant l^{\prime}-3} m s P\left(n+l+l^{\prime}-3-2 i, 1+i\right)\right) \\
\cong & M_{m}\left(l, 0, \alpha q^{1-l}(l)_{q}\right) \otimes M_{s}\left(l^{\prime}, 0, \eta q^{1-l^{\prime}}\left(l^{\prime}\right)_{q}\right) \\
& \oplus\left(\oplus_{i=0}^{c\left(n+l-l^{\prime}\right)} m s P\left(l^{\prime}-l-1+2 i, l-i\right)\right) \\
& \oplus\left(\oplus_{c\left(l+l^{\prime}-1\right) \leqslant i \leqslant l^{\prime}-2} m s P\left(n+l+l^{\prime}-1-2 i, i\right)\right) .
\end{aligned}
$$

On the other hand, by Proposition 3.1, Theorems 3.3 and 3.5, one can check that

$$
\begin{aligned}
& \left(\oplus_{i=1}^{c\left(n+l-l^{\prime}+1\right)} P\left(l^{\prime}-l-2+2 i, l-i\right) \otimes V(2,0)\right) \\
& \oplus\left(\oplus_{c\left(l+l^{\prime}-2\right) \leqslant i \leqslant l^{\prime}-2} P\left(n+l+l^{\prime}-2-2 i, i\right) \otimes V(2,0)\right) \\
\cong & \left(\oplus_{i=1}^{c\left(n+l-l^{\prime}\right)} P\left(l^{\prime}-l-1+2 i, l-i\right)\right) \\
& \oplus\left(\oplus_{i=0}^{c\left(n+l-l^{\prime}\right)} P\left(l^{\prime}-l-1+2 i, l-i\right)\right) \\
& \oplus\left(\oplus_{i=c\left(l+l^{\prime}-1\right)}^{l^{\prime}-1} P\left(n+l+l^{\prime}-1-2 i, i\right)\right) \\
& \oplus\left(\oplus_{c\left(l+l^{\prime}-1\right) \leqslant i \leqslant l^{\prime}-2} P\left(n+l+l^{\prime}-1-2 i, i\right)\right) .
\end{aligned}
$$

Hence it follows from Krull-Schmidt Theorem that

$$
\begin{aligned}
& M_{m}\left(l, 0, \alpha q^{1-l}(l)_{q}\right) \otimes M_{s}\left(l^{\prime}, 0, \eta q^{1-l^{\prime}}\left(l^{\prime}\right)_{q}\right) \\
\cong & \left(\oplus_{i=1}^{c\left(n+l-l^{\prime}\right)} m s P\left(l^{\prime}-l-1+2 i, l-i\right)\right) \\
& \oplus\left(\bigoplus_{i=c\left(l+l^{\prime}-1\right)}^{l^{\prime}-1} m s P\left(n+l+l^{\prime}-1-2 i, i\right)\right),
\end{aligned}
$$

as desired. If $l^{\prime}>2$ and $l=l^{\prime}-1$, then by the induction hypothesis, and Theorems 3.3 and 3.5, a similar argument as above shows that

$$
M_{m}\left(l, 0, \alpha q^{1-l}(l)_{q}\right) \otimes M_{s}\left(l^{\prime}, 0, \eta q^{1-l^{\prime}}\left(l^{\prime}\right)_{q}\right) \cong\left(\oplus_{i=1}^{c(n-1)} m s P(2 i, l-i)\right) \oplus m s V(n, l) .
$$

This completes the proof.

Corollary 5.17. Let $1 \leqslant l \leqslant l^{\prime}<n, r, r^{\prime} \in \mathbb{Z}_{n}, \alpha, \eta \in \mathbb{P}^{1}(k)$ and $s, m \geqslant 1$. Assume that $\alpha q^{1-l^{\prime}}\left(l^{\prime}\right)_{q} \neq \eta q^{1-l}(l)_{q}$. Then

$$
\begin{aligned}
& M_{m}(l, r, \alpha) \otimes M_{s}\left(l^{\prime}, r^{\prime}, \eta\right) \\
\cong & \left(\oplus_{i=1}^{c\left(n-l^{\prime}+l\right)} m s P\left(l^{\prime}-l-1+2 i, r+r^{\prime}+l-i\right)\right) \\
& \oplus\left(\oplus_{c\left(l+l^{\prime}-1\right) \leqslant i \leqslant l^{\prime}-1} m s P\left(n+l+l^{\prime}-1-2 i, r+r^{\prime}+i\right)\right) .
\end{aligned}
$$

Proof. It follows from Proposition 5.16.

Now we investigate $M_{m}(l, r, \alpha) \otimes M_{s}\left(l^{\prime}, r^{\prime}, \eta\right)$ for $\alpha q^{1-l^{\prime}}\left(l^{\prime}\right)_{q}=\eta q^{1-l}(l)_{q}$. We only need to consider the case $m \geqslant s$ since $M_{m}(l, r, \alpha) \otimes M_{s}\left(l^{\prime}, r^{\prime}, \eta\right) \cong M_{s}\left(l^{\prime}, r^{\prime}, \eta\right) \otimes$ $M_{m}(l, r, \alpha)$. 
Lemma 5.18. Let $M$ be an indecomposable module with $\operatorname{rl}(M)=2$.

(1) If $M$ is of $(s+1, s)$-type, then $M$ contains no submodules of $(i+1, i)$-type for any $s>i \geqslant 1$, and consequently, $M$ contains no proper submodule $N$ with $l(N / \operatorname{soc}(N))>l(N)$.

(2) If $M$ is of $(s, s)$-type, then $M$ contains no submodules of $(i+1, i)$-type, and consequently, $M$ contains no submodule $N$ with $l(N / \operatorname{soc}(N))>l(N)$.

Proof. It follows from [6, Lemma 4.3] and [7, Proposition 3.3]. It also can be shown by an argument similar to the proof of [6. Lemma 4.3].

Lemma 5.19. Let $s \geqslant 1$ and $M$ be an indecomposable module of $(s, s)$-type. Then $M$ can be embedded into an indecomposable module of $(s+1, s)$-type.

Proof. It is similar to [8, Lemma 3.28] by using Lemma [5.18,

Lemma 5.20. Let $r, r^{\prime} \in \mathbb{Z}_{n}, \eta \in \mathbb{P}^{1}(k)$ and $s \geqslant 1$. Then $M_{s}(1, r, \eta) \otimes M_{s}\left(1, r^{\prime}, \eta\right)$ contains a submodule isomorphic to $M_{s}\left(n-1, r+r^{\prime}+1,-\eta q\right)$.

Proof. By Lemma 3.8 it is enough to show the lemma for $r=r^{\prime}=0$. Assume that $\eta \in k$ and let $M=M_{s}(1,0, \eta) \otimes M_{s}(1,0, \eta)$. By Lemma 3.10, there is a standard basis $\left\{v_{i, j} \mid 1 \leqslant i \leqslant n, 1 \leqslant j \leqslant s\right\}$ in $M_{s}(1,0, \eta)$ such that

$$
\begin{aligned}
& a v_{i, j}= \begin{cases}v_{i+1, j}, \quad 1 \leqslant i<n, & b v_{i, j}=q^{i} v_{i, j}, \\
0, & i=n,\end{cases} \\
& d v_{i, j}=\left\{\begin{array}{ll}
v_{n, j-1}+\eta q v_{n, j}, & i=1, \\
\alpha_{i-1}(n-1) v_{i-1, j}, & 1<i \leqslant n-1, \\
0, & i=n,
\end{array} \quad c v_{i, j}=q^{i} v_{i, j},\right.
\end{aligned}
$$

where $1 \leqslant i \leqslant n, 1 \leqslant j \leqslant s$ and $v_{n, 0}=0$. Then $\left\{v_{i, j} \otimes v_{l, m} \mid 1 \leqslant i, l \leqslant n, 1 \leqslant j, m \leqslant\right.$ $s\}$ is a basis of $M$. For $1 \leqslant i \leqslant n$ and $1 \leqslant j \leqslant s$, let $u_{i, j} \in M$ be defined by

$$
u_{1, j}=(-q)^{j} \sum_{l=1}^{j} \sum_{m=1}^{n-1}(-1)^{m-1} q^{-\frac{m(m-1)}{2}} v_{m, l} \otimes v_{n-m, j+1-l}
$$

and

$$
u_{i, j}=(-q)^{j} \sum_{l=1}^{j}\left(v_{i-1, l} \otimes v_{n, j+1-l}-v_{n, l} \otimes v_{i-1, j+1-l}\right)
$$

for $2 \leqslant i \leqslant n$. Then $\left\{u_{i, j} \mid 1 \leqslant i \leqslant n, 1 \leqslant j \leqslant s\right\}$ are linearly independent over $k$. A tedious but standard verification shows that $N=\operatorname{span}\left\{u_{i, j} \mid 1 \leqslant i \leqslant n, 1 \leqslant j \leqslant s\right\}$ is a submodule of $M$, and $N \cong M_{s}(n-1,1,-\eta q)$ by Lemma 3.10

For $\eta=\infty$, using Lemma 3.9. one can similarly show that $M_{s}(1,0, \infty) \otimes M_{s}(1,0, \infty)$ contains a submodule isomorphic to $M_{s}(n-1,1, \infty)$. This completes the proof.

Lemma 5.21. Let $r, r^{\prime} \in \mathbb{Z}_{n}, \eta \in \mathbb{P}^{1}(k)$ and $m \geqslant s \geqslant 1$. Then

$$
\begin{aligned}
& M_{m}(1, r, \eta) \otimes M_{s}\left(1, r^{\prime}, \eta\right) \\
\cong & M_{s}\left(1, r+r^{\prime}, \eta\right) \oplus M_{s}\left(n-1, r+r^{\prime}+1,-\eta q\right) \\
& \oplus(m-1) s P\left(1, r+r^{\prime}\right) \oplus\left(\oplus_{i=1}^{c(n-2)} m s P\left(2 i+1, r+r^{\prime}-i\right)\right) .
\end{aligned}
$$


Proof. It is enough to show the lemma for $r=r^{\prime}=0$. We only consider the case that $m$ is odd since the proof is similar for $m$ being even.

Assume that $m$ is odd. Then by Lemma 5.19, there is an exact sequence

$$
0 \rightarrow M_{m}(1,0, \eta) \rightarrow \Omega^{m} V(1,0) \rightarrow V(n-1,1) \rightarrow 0
$$

Applying $\otimes M_{s}(1,0, \eta)$ to the above sequence, one gets the following exact sequence

$$
\begin{aligned}
0 \rightarrow M_{m}(1,0, \eta) \otimes M_{s}(1,0, \eta) & \stackrel{\sigma}{\rightarrow} \Omega^{m} V(1,0) \otimes M_{s}(1,0, \eta) \\
& \rightarrow V(n-1,1) \otimes M_{s}(1,0, \eta) \rightarrow 0 .
\end{aligned}
$$

By [7, Theorem 3.10(2)], $M_{m}(1,0, \eta)$ contains a unique submodule $M$ of $(s, s)$-type, and $M \cong M_{s}(1,0, \eta)$. From Lemma 5.20 one knows that $M \otimes M_{s}(1,0, \eta)$ contains a submodule isomorphic to $M_{s}(n-1,1,-\eta q)$. It follows that $M_{m}(1,0, \eta) \otimes M_{s}(1,0, \eta)$ contains a submodule $N$ isomorphic to $M_{s}(n-1,1,-\eta q)$. From Proposition 5.6, $\Omega^{m} V(1,0) \otimes M_{s}(1,0, \eta)$ contains submodules $M^{\prime}$ and $P$ with $M^{\prime} \cong M_{s}(n-1,1,-\eta q)$ and $P \cong m s P(1,0) \oplus\left(\oplus_{i=1}^{c(n-2)}(m+1) s P(2 i+1,-i)\right)$ such that $\Omega^{m} V(1,0) \otimes$ $M_{s}(1,0, \eta)=P \oplus M^{\prime}$. Since $\sigma$ is a monomorphism, $\sigma(N) \cong N \cong M_{s}(n-1,1,-\eta q)$, and hence $\operatorname{soc}(\sigma(N)) \cong s V(n-1,1)$. However, $\operatorname{soc}(P) \cong m s V(1,0) \oplus\left(\oplus_{i=1}^{c(n-2)}(m+\right.$ $1) s V(2 i+1,-i))$ since $\operatorname{soc}(P(l, r)) \cong V(l, r)$ for all $1 \leqslant l \leqslant n$ and $r \in \mathbb{Z}$. It follows that the sum $P+\sigma(N)$ is direct, and so $\Omega^{m} V(1,0) \otimes M_{s}(1,0, \eta)=P \oplus M^{\prime}=P \oplus \sigma(N)$ by comparing their lengths. Hence we have the following exact sequence

$$
0 \rightarrow M_{m}(1,0, \eta) \otimes M_{s}(1,0, \eta) \stackrel{\sigma}{\rightarrow} P \oplus \sigma(N) \stackrel{f}{\rightarrow} V(n-1,1) \otimes M_{s}(1,0, \eta) \rightarrow 0 .
$$

Since $f$ is an epimorphism and $f(\sigma(N))=0,\left.f\right|_{P}: P \rightarrow V(n-1,1) \otimes M_{s}(1,0, \eta)$ is an epimorphism. By Theorem 3.16, we have

$$
\begin{aligned}
& V(n-1,1) \otimes M_{s}(1,0, \eta) \\
\cong & M_{s}(n-1,1,-\eta q) \oplus\left(\oplus_{i=c(n-1)}^{n-2} s P(2 n-1-2 i, i+1)\right) \\
\cong & M_{s}(n-1,1,-\eta q) \oplus\left(\oplus_{i=1}^{c(n-2)} s P(2 i+1,-i)\right) .
\end{aligned}
$$

Note that $s P(1,0) \cong P\left(M_{s}(n-1,1,-\eta q)\right)$ and $\Omega M_{s}(n-1,1,-\eta q) \cong M_{s}(1,0, \eta)$. Hence we have

$$
P \cong P\left(V(n-1,1) \otimes M_{s}(1,0, \eta)\right) \oplus(m-1) s P(1,0) \oplus\left(\oplus_{i=1}^{c(n-2)} m s P(2 i+1,-i)\right) .
$$

It follows from Lemma 5.1 that

$$
\operatorname{Ker}\left(\left.f\right|_{P}\right) \cong M_{s}(1,0, \eta) \oplus(m-1) s P(1,0) \oplus\left(\oplus_{i=1}^{c(n-2)} m s P(2 i+1,-i)\right),
$$

and so

$$
\begin{aligned}
& M_{m}(1,0, \eta) \otimes M_{s}(1,0, \eta) \cong \operatorname{Ker}(f)=\operatorname{Ker}\left(\left.f\right|_{P}\right) \oplus \sigma(N) \\
\cong & M_{s}(1,0, \eta) \oplus M_{s}(n-1,1,-\eta q) \\
& \oplus(m-1) s P(1,0) \oplus\left(\oplus_{i=1}^{c(n-2)} m s P(2 i+1,-i)\right)
\end{aligned}
$$


Theorem 5.22. Let $1 \leqslant l, l^{\prime}<n, r, r^{\prime} \in \mathbb{Z}_{n}, \eta \in \mathbb{P}^{1}(k)$ and $m \geqslant s \geqslant 1$. Assume that $l+l^{\prime} \leqslant n$, and let $l_{1}=\min \left\{l, l^{\prime}\right\}$ and $l_{2}=\max \left\{l, l^{\prime}\right\}$. Then

$$
\begin{aligned}
& M_{m}\left(l, r, \eta q^{1-l}(l)_{q}\right) \otimes M_{s}\left(l^{\prime}, r^{\prime}, \eta q^{1-l^{\prime}}\left(l^{\prime}\right)_{q}\right) \\
\cong & \left(\oplus_{i=0}^{l_{1}-1} M_{s}\left(l+l^{\prime}-1-2 i, r+r^{\prime}+i, \eta q^{2 i-l-l^{\prime}+2}\left(l+l^{\prime}-1-2 i\right)_{q}\right)\right) \\
& \oplus\left(\oplus_{i=l_{2}}^{l+l^{\prime}-1} M_{s}\left(n+l+l^{\prime}-1-2 i, r+r^{\prime}+i,-\eta q\left(2 i-l-l^{\prime}+1\right)_{q}\right)\right) \\
& \oplus\left(\oplus_{i=0}^{l_{1}-1}(m-1) s P\left(l+l^{\prime}-1-2 i, r+r^{\prime}+i\right)\right) \\
& \oplus\left(\oplus_{1 \leqslant i \leqslant c\left(n-l-l^{\prime}\right)} m s P\left(l+l^{\prime}-1+2 i, r+r^{\prime}-i\right)\right) \\
& \oplus\left(\oplus_{c\left(l+l^{\prime}-1\right) \leqslant i \leqslant l_{2}-1} m s P\left(n+l+l^{\prime}-1-2 i, r+r^{\prime}+i\right)\right) .
\end{aligned}
$$

Proof. It is enough to show the theorem for $r=r^{\prime}=0$. We prove it by the induction on $l+l^{\prime}$. For $l+l^{\prime}=2$, it follows from Lemma 5.21. Now assume that $l+l^{\prime}>2$. Here we only consider the case of $l=l^{\prime}$ since the proof are similar for the other cases: $l<l^{\prime}-1, l=l^{\prime}-1, l>l^{\prime}+1$ and $l=l^{\prime}+1$. Suppose $l=l^{\prime}$. Then $l \geqslant 2$. By the induction hypothesis, applying $V(2,0) \otimes$ and then using Proposition 3.1. Theorems 3.3, 3.5 and 3.16, a tedious but standard computation shows that

$$
\begin{aligned}
& V(2,0) \otimes M_{m}\left(l-1,0, \eta q^{2-l}(l-1)_{q}\right) \otimes M_{s}\left(l, 0, \eta q^{1-l}(l)_{q}\right) \\
\cong & \left(\oplus_{i=0}^{l-2}\left(V(2,0) \otimes M_{s}\left(2 l-2-2 i, i, \eta q^{2 i-2 l+3}(2 l-2-2 i)_{q}\right)\right)\right. \\
& \oplus\left(\oplus_{i=l}^{2 l-2} V(2,0) \otimes M_{s}\left(n+2 l-2-2 i, i,-\eta q(2 i-2 l+2)_{q}\right)\right) \\
& \oplus\left(\oplus_{i=0}^{l-2}(m-1) s V(2,0) \otimes P(2 l-2-2 i, i)\right) \\
& \oplus\left(\oplus_{i=1}^{c(n-2 l+1)} m s V(2,0) \otimes P(2 l-2+2 i,-i)\right) \oplus m s V(2,0) \otimes V(n, l-1) \\
\cong & \left(\oplus_{i=0}^{l-2} M_{s}\left(2 l-1-2 i, i, \eta q^{2 i-2 l+2}(2 l-2 i-1)_{q}\right)\right) \\
& \oplus\left(\oplus_{i=1}^{l-1} M_{s}\left(2 l-1-2 i, i, \eta q^{2 i-2 l+2}(2 l-2 i-1)_{q}\right)\right) \\
& \oplus\left(\oplus_{i=l}^{2 l-2} M_{s}\left(n+2 l-1-2 i, i,-\eta q(2 i-2 l+1)_{q}\right)\right) \\
& \oplus\left(\oplus_{i=l+1}^{2 l-1} M_{s}\left(n+2 l-1-2 i, i,-\eta q(2 i-2 l+1)_{q}\right)\right) \\
& \oplus\left(\oplus_{i=0}^{l-2}(m-1) s P(2 l-1-2 i, i)\right) \oplus\left(\oplus_{i=1}^{l-1}(m-1) s P(2 l-1-2 i, i)\right) \\
& \oplus\left(\oplus_{1 \leqslant i \leqslant c(n-2 l)} m s P(2 l-1+2 i,-i)\right) \\
& \oplus\left(\oplus_{i=0}^{c(n-2 l)} m s P(2 l-1+2 i,-i)\right) \oplus m s P(n-1, l) .
\end{aligned}
$$

If $l>2$, then by Theorem 3.16 and the induction hypothesis, we have

$$
\begin{aligned}
& V(2,0) \otimes M_{m}\left(l-1,0, \eta q^{2-l}(l-1)_{q}\right) \otimes M_{s}\left(l, 0, \eta q^{1-l}(l)_{q}\right) \\
\cong & M_{m}\left(l, 0, \eta q^{1-l}(l)_{q}\right) \otimes M_{s}\left(l, 0, \eta q^{1-l}(l)_{q}\right) \\
& \oplus M_{m}\left(l-2,1, \eta q^{3-l}(l-2)_{q}\right) \otimes M_{s}\left(l, 0, \eta q^{1-l}(l)_{q}\right) \\
\cong & M_{m}\left(l, 0, \eta q^{1-l}(l)_{q}\right) \otimes M_{s}\left(l, 0, \eta q^{1-l}(l)_{q}\right) \\
& \oplus\left(\oplus_{i=0}^{l-3} M_{s}\left(2 l-3-2 i, i+1, \eta q^{2 i-2 l+4}(2 l-3-2 i)_{q}\right)\right) \\
& \oplus\left(\oplus_{i=l}^{2 l-3} M_{s}\left(n+2 l-3-2 i, i+1,-\eta q(2 i-2 l+3)_{q}\right)\right) \\
& \oplus\left(\oplus_{i=0}^{l-3}(m-1) s P(2 l-3-2 i, i+1)\right) \\
& \left.\oplus\left(\oplus_{i=1}^{c(n-2 l+2)} m s P(2 l-3+2 i, 1-i)\right) \oplus m s P(n-1, l)\right) \\
\cong & M_{m}\left(l, 0, \eta q^{1-l}(l)_{q}\right) \otimes M_{s}\left(l, 0, \eta q^{1-l}(l)_{q}\right) \\
& \oplus\left(\oplus_{1 \leqslant i \leqslant l-2} M_{s}\left(2 l-1-2 i, i, \eta q^{2 i-2 l+2}(2 l-1-2 i)_{q}\right)\right) \\
& \oplus\left(\oplus_{l+1 \leqslant i \leqslant 2 l-2} M_{s}\left(n+2 l-1-2 i, i,-\eta q(2 i-2 l+1)_{q}\right)\right) \\
& \oplus\left(\oplus_{1 \leqslant i \leqslant l-2}(m-1) s P(2 l-1-2 i, i)\right) \\
& \left.\oplus\left(\oplus_{i=0}^{c(n-2 l)} m s P(2 l-1+2 i,-i)\right) \oplus m s P(n-1, l)\right) .
\end{aligned}
$$


If $l=2$, by Lemma 3.11 and Theorem 3.17, one can similarly show the above isomorphism. Hence by Krull-Schmidt Theorem, we have

$$
\begin{aligned}
& M_{m}\left(l, 0, \eta q^{1-l}(l)_{q}\right) \otimes M_{s}\left(l, 0, \eta q^{1-l}(l)_{q}\right) \\
\cong & \left(\oplus_{i=0}^{l-1} M_{s}\left(2 l-1-2 i, i, \eta q^{2 i-2 l+2}(2 l-2 i-1)_{q}\right)\right) \\
& \oplus\left(\oplus_{i=l}^{2 l-1} M_{s}\left(n+2 l-1-2 i, i,-\eta q(2 i-2 l+1)_{q}\right)\right) \\
& \oplus\left(\oplus_{i=0}^{l-1}(m-1) s P(2 l-1-2 i, i)\right) \\
& \oplus\left(\oplus_{1 \leqslant i \leqslant c(n-2 l)} m s P(2 l-1+2 i,-i)\right) .
\end{aligned}
$$

This completes the proof.

Corollary 5.23. Let $1 \leqslant l, l^{\prime}<n, r, r^{\prime} \in \mathbb{Z}_{n}, \alpha, \eta \in \mathbb{P}^{1}(k)$ and $m \geqslant s \geqslant 1$. Assume that $l+l^{\prime} \leqslant n$, and let $l_{1}=\min \left\{l, l^{\prime}\right\}$ and $l_{2}=\max \left\{l, l^{\prime}\right\}$. If $\alpha q^{1-l^{\prime}}\left(l^{\prime}\right)_{q}=\eta q^{1-l}(l)_{q}$, then

$$
\begin{aligned}
& M_{m}(l, r, \alpha) \otimes M_{s}\left(l^{\prime}, r^{\prime}, \eta\right) \\
& \cong\left(\oplus_{i=0}^{l_{1}-1} M_{s}\left(l+l^{\prime}-1-2 i, r+r^{\prime}+i, \eta q^{2 i-l+1} \frac{\left(l+l^{\prime}-1-2 i\right)_{q}}{\left(l^{\prime}\right)_{q}}\right)\right) \\
& \oplus\left(\oplus_{i=l_{2}}^{l+l^{\prime}-1} M_{s}\left(n+l+l^{\prime}-1-2 i, r+r^{\prime}+i,-\eta q^{l^{\prime}} \frac{\left.\left(2 i-l-l^{\prime}+1\right)_{q}\right)}{\left(l^{\prime}\right)_{q}}\right)\right) \\
& \oplus\left(\oplus_{i=0}^{l_{1}-1}(m-1) s P\left(l+l^{\prime}-1-2 i, r+r^{\prime}+i\right)\right) \\
& \oplus\left(\oplus_{1 \leqslant i \leqslant c\left(n-l-l^{\prime}\right)} m s P\left(l+l^{\prime}-1+2 i, r+r^{\prime}-i\right)\right) \\
& \oplus\left(\oplus_{c\left(l+l^{\prime}-1\right) \leqslant i \leqslant l_{2}-1} m s P\left(n+l+l^{\prime}-1-2 i, r+r^{\prime}+i\right)\right) \text {. }
\end{aligned}
$$

Proof. It follows from Theorem 5.22 .

Corollary 5.24. Let $1 \leqslant l, l^{\prime}<n, r, r^{\prime} \in \mathbb{Z}_{n}, \eta \in \mathbb{P}^{1}(k)$ and $m \geqslant s \geqslant 1$. Assume that $t=l+l^{\prime}-(n+1) \geqslant 0$. Let $l_{1}=\min \left\{l, l^{\prime}\right\}$ and $l_{2}=\max \left\{l, l^{\prime}\right\}$. Then

$$
\begin{aligned}
& M_{m}\left(l, r, \eta q^{1-l}(l)_{q}\right) \otimes M_{s}\left(l^{\prime}, r^{\prime}, \eta q^{1-l^{\prime}}\left(l^{\prime}\right)_{q}\right) \\
\cong & \left(\oplus_{i=t+1}^{l_{1}-1} M_{s}\left(l+l^{\prime}-1-2 i, r+r^{\prime}+i, \eta q^{2 i-l-l^{\prime}+2}\left(l+l^{\prime}-1-2 i\right)_{q}\right)\right) \\
& \oplus\left(\oplus_{i=l_{2}}^{n-1} M_{s}\left(n+l+l^{\prime}-1-2 i, r+r^{\prime}+i,-\eta q\left(2 i-l-l^{\prime}+1\right)_{q}\right)\right) \\
& \oplus\left(\oplus_{i=t+1}^{l_{1}-1}(m-1) s P\left(l+l^{\prime}-1-2 i, r+r^{\prime}+i\right)\right) \\
& \oplus\left(\oplus_{i=c(t)}^{t} m s P\left(l+l^{\prime}-1-2 i, r+r^{\prime}+i\right)\right) \\
& \oplus\left(\oplus_{c\left(l+l^{\prime}-1\right) \leqslant i \leqslant l_{2}-1} m s P\left(n+l+l^{\prime}-1-2 i, r+r^{\prime}+i\right)\right) .
\end{aligned}
$$

Proof. It is enough to show the corollary for $r=r^{\prime}=0$. Since $1 \leqslant l, l^{\prime}<n$ and $l+l^{\prime}>n, 1 \leqslant n-l, n-l^{\prime}<n$ and $(n-l)+\left(n-l^{\prime}\right)<n$. Hence by Theorem 5.22 . we have

$$
\begin{aligned}
& M_{m}\left(n-l, 1,-\eta q(l)_{q}\right) \otimes M_{s}\left(n-l^{\prime}, 1,-\eta q\left(l^{\prime}\right)_{q}\right) \\
\cong & M_{m}\left(n-l, 1, \eta q^{1+l}(n-l)_{q}\right) \otimes M_{s}\left(l^{\prime}, 1, \eta q^{1+l^{\prime}}\left(n-l^{\prime}\right)_{q}\right) \\
\cong & \left(\oplus_{i=0}^{n-l_{2}-1} M_{s}\left(2 n-l-l^{\prime}-1-2 i, i+2, \eta q^{2 i+l+l^{\prime}+2}\left(2 n-l-l^{\prime}-1-2 i\right)_{q}\right)\right) \\
& \oplus\left(\oplus_{i=n-l_{1}-1}^{2 n-l-1} M_{s}\left(3 n-l-l^{\prime}-1-2 i, i+2,-\eta q\left(2 i-2 n+l+l^{\prime}+1\right)_{q}\right)\right) \\
& \oplus\left(\oplus_{i=0}^{n-l_{2}-1}(m-1) s P\left(2 n-l-l^{\prime}-1-2 i, 2+i\right)\right) \\
& \oplus\left(\oplus_{i=1}^{c\left(l+l^{\prime}-n\right)} m s P\left(2 n-l-l^{\prime}-1+2 i, 2-i\right)\right) \\
& \oplus\left(\oplus_{c\left(2 n-l-l^{\prime}-1\right) \leqslant i \leqslant n-l_{1}-1} m s P\left(3 n-l-l^{\prime}-1-2 i, 2+i\right)\right) .
\end{aligned}
$$

Then by applying the duality $(-)^{*}$ to the above isomorphism, the corollary follows from Lemmas 3.12 and 3.13 . 
Corollary 5.25. Let $1 \leqslant l, l^{\prime}<n, r, r^{\prime} \in \mathbb{Z}_{n}, \alpha, \eta \in \mathbb{P}^{1}(k)$ and $m \geqslant s \geqslant 1$. Assume that $t=l+l^{\prime}-(n+1) \geqslant 0$. Let $l_{1}=\min \left\{l, l^{\prime}\right\}$ and $l_{2}=\max \left\{l, l^{\prime}\right\}$. If $\alpha q^{1-l^{\prime}}\left(l^{\prime}\right)_{q}=\eta q^{1-l}(l)_{q}$, then

$$
\begin{aligned}
& M_{m}(l, r, \alpha) \otimes M_{s}\left(l^{\prime}, r^{\prime}, \eta\right) \\
\cong & \left(\oplus_{i=t+1}^{l_{1}-1} M_{s}\left(l+l^{\prime}-1-2 i, r+r^{\prime}+i, \eta q^{2 i-l+1} \frac{\left(l+l^{\prime}-1-2 i\right)_{q}}{\left(l^{\prime}\right)_{q}}\right)\right) \\
& \oplus\left(\oplus_{i=l_{2}}^{n-1} M_{s}\left(n+l+l^{\prime}-1-2 i, r+r^{\prime}+i,-\eta q^{l^{\prime}} \frac{\left(2 i-l l^{\prime}+1\right)_{q}}{\left(l^{\prime}\right)_{q}}\right)\right) \\
& \oplus\left(\oplus_{i=t+1}^{l_{1}-1}(m-1) s P\left(l+l^{\prime}-1-2 i, r+r^{\prime}+i\right)\right) \\
& \oplus\left(\oplus_{i=c(t)}^{t} m s P\left(l+l^{\prime}-1-2 i, r+r^{\prime}+i\right)\right) \\
& \oplus\left(\oplus_{c\left(l+l^{\prime}-1\right) \leqslant i \leqslant l_{2}-1} m s P\left(n+l+l^{\prime}-1-2 i, r+r^{\prime}+i\right)\right) .
\end{aligned}
$$

Proof. It follows from Corollary 5.24

\section{ACKNOWLEDGMENTS}

The authors would like to show their gratitude to the referee for his/her helpful suggestions. This work is supported by NSF of China (No. 11571298).

\section{REFERENCES}

[1] J. Andrea, A trace-like invariant for representations of Hopf algebras, Comm. Algebra 38 (2010), 3456-3468.

[2] M. Auslander, I. Reiten and S. O. Smalø, Representation Theory of Artin Algebras, Cambridge Univ. Press, Cambridge, 1995.

[3] V. Chari and A. Premet, Indecomposable restricted representations of quantum $s l_{2}$, Publ. RIMS. Kyoto Univ. 30 (1994), 335-352.

[4] H. X. Chen, A class of noncommutative and noncocommutative Hopf algebras-the quantum version, Comm. Algebra 27 (1999), 5011-5023.

[5] H. X. Chen, Irreducible representations of a class of quantum doubles, J. Algebra 225 (2000), 391-409.

[6] H. X. Chen, Finite-dimensional representations of a quantum double, J. Algebra 251 (2002), 751-789.

[7] H. X. Chen, Representations of a class of Drinfeld's doubles, Comm. Algebra 33 (2005), 2809-2825.

[8] H. X. Chen, The Green ring of Drinfeld double $D\left(H_{4}\right)$, Algebr. Represent. Theor. 17 (2014), $1457-1483$.

[9] X. W. Chen, H. L. Huang, Y. Ye and P. Zhang, Monomial Hopf algebras, J. Algebra 275 (2004), 212-232.

[10] H. X. Chen, F. Van Oystaeyen and Y. H. Zhang, The Green rings of Taft algebras, Proc. Amer. Math. Soc. 142 (2014), 765-775.

[11] W. Chin and L. Krop, Representation theory of liftings of quantum planes, J. Algebra Appl. 8 (2009), 259-287.

[12] C. Cibils, A quiver quantum group, Commun. Math. Phys. 157 (1993), 459-477.

[13] K. Erdmann, E. L. Green, N. Snashall and Taillefer, Representation theory of the Drinfeld doubles of a family of Hopf algebras, J. Pure Appl. Algebra 204 (2006), 413-454.

[14] E. Gunnlaugsdóttir, Monoidal structure of the category of $\mathfrak{u}_{q}^{+}$-modules, Linear Algebra and its Applications 365 (2003), 183-199.

[15] H. L. Huang, H. X. Chen and P. Zhang, Generalized Taft algebras, Algebra Colloq. 11 (2004), 313-320.

[16] C. Kassel, Quantum groups, Springer-Verlag, New York, 1995.

[17] H. Kondo and Y. Saito, Indecomposable decomposition of tensor products of modules over the restricted quantum universal enveloping algebra associated to $\mathfrak{s l}_{2}$, J. Algebra 330 (2011), 103-129. 
[18] L. Krop and D. E. Radford, Finite-dimensional Hopf algebras of rank one in characteristic zero, J. Algebra 302 (2006), 214-230.

[19] L. Krop and D. E. Radford, Representations of pointed Hopf algebras and their Drinfeld quantum doubles, J. Algebra 321 (2009), 2567-2603.

[20] Y. Li and N. Hu, The Green rings of the 2-rank Taft algebra and its two relatives twisted, J. Algebra 410 (2014), 1-35.

[21] L. B. Li and Y. H. Zhang, The Green rings of the generalized Taft Hopf algebras, Contemporary Mathematics 585 (2013), 275-288.

[22] M. Lorenz, Representations of finite-dimensional Hopf algebras, J. Algebra 188 (1997), 476505.

[23] S. Montgomery, Hopf Algebras and their actions on rings, CBMS Series in Math., Vol. 82, Am. Math. Soc., Providence, 1993.

[24] U. Oberst and H.-J. Schneider, Uber untergruppen endlicher algebraischer gruppen, Manuscripta Math. 8 (1973), 217-241.

[25] A. A. Premet, The Green ring of the simple three-dimensional Lie $p$-algbera, Izv. Vuzov. Math. 10 (1991), 56-67. (in Russian)

[26] D. E. Radford, On the coradical of a finite-dimensional Hopf algebra, Proc. Amer. Math. Soc. 53 (1975), 9-15.

[27] D. E. Radford, Minimal quasitriangular Hopf algebras, J. Algebra 175 (1993), 285-315.

[28] D. E. Radford and H. -J. Schneider, On the simple representations of generalized quantum groups and quantum doubles, J. Algebra 319 (2008), 3689-3731.

[29] R. Suter, Modules for $\mathfrak{U}_{q}\left(\mathfrak{s l}_{2}\right)$, Comm. Math. Phys. 163 (1994), 359-393.

[30] M. E. Sweedler, Hopf Algebras, Benjamin, New York, 1969.

[31] E. J. Taft, The order of the antipode of a finite-dimensional Hopf algebra, Proc. Nat. Acad. Sci. USA 68 (1971), 2631-2633.

[32] S. J. Witherspoon, The representation ring of the quantum double of a finite group, J. Algebra 179 (1996), 305-329.

[33] Y. Zhang, F. Wu, L. Liu and H. X. Chen, Grothendieck groups of a class of quantum doubles, Algebra Colloq. 15(2008), 431-448.

School of Mathematical Science, Yangzhou University, Yangzhou 225002, China

E-mail address: hxchen@yzu.edu.cn

School of Mathematical Science, Yangzhou University, Yangzhou 225002, China

E-mail address: esmailhassan313@yahoo.com

School of Mathematical Science, Yangzhou University, Yangzhou 225002, China

E-mail address: 997749901@qq.com 